\title{
STREAMFLOW AND SELECTED PRECIPITATION DATA FOR YUCCA MOUNTAIN AND VICINITY, NYE COUNTY, NEVADA, WATER YEARS 1983-85
}

By Marilyn E. Pabst, David A. Beck, Patrick A. Glancy, and Jeffrey A. Johnson

U.S. GEOLOGICAL SURVEY

Open-File Report 93-438

Prepared in cooperation with the NEVADA OPERATIONS OFFICE

U.S. DEPARTMENT OF ENERGY, under

INTERAGENCY AGREEMENT DE-AI08-78ET4482

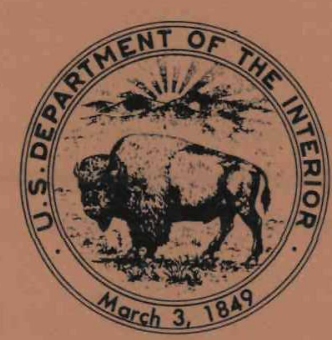

Carson City, Nevada 1993 
The following number is for U.S. Department of Energy Office of Civilian Radioactive Waste Management (OCRWM) records management purposes only and should not be used when ordering this publication: ACCESSION NUMBER: NNA.931019.0043 


\section{DISCLAIMER}

This report was prepared as an account of work sponsored by an agency of the United States Government. Neither the United States Government nor any agency thereof, nor any of their employees, make any warranty, express or implied, or assumes any legal liability or responsibility for the accuracy, completeness, or usefulness of any information, apparatus, product, or process disclosed, or represents that its use would not infringe privately owned rights. Reference herein to any specific commercial product, process, or service by trade name, trademark, manufacturer, or otherwise does not necessarily constitute or imply its endorsement, recommendation, or favoring by the United States Government or any agency thereof. The views and opinions of authors expressed herein do not necessarily state or reflect those of the United States Government or any agency thereof. 


\section{DISCLAIMER}

Portions of this document may be illegible in electronic image products. Images are produced from the best available original document. 


\section{STREAMFLOW AND SELECTED PRECIPITATION DATA FOR YUCCA MOUNTAIN AND VICINITY, NYE COUNTY, NEVADA, WATER YEARS 1983-85}

U.S. GEOLOGICAL SURVEY

Open-File Report $93-438$

Prepared in cooperation with the NEVADA OPERATIONS OFFICE

U.S. DEPARTMENT OF ENERGY, under

INTERAGENCY AGREEMENT DE-AI08-78ET4482
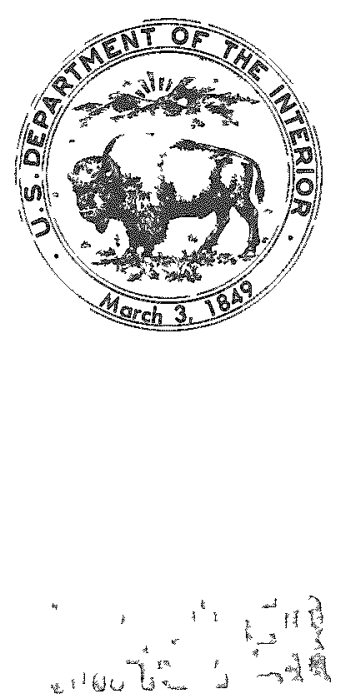


\title{
U.S. DEPARTMENT OF THE INTERIOR
}

BRUCE BABBITT, Secretary

\author{
U.S. GEOLOGICAL SURVEY
}

Robert M Hirsch, Acting Director

Any use of trade, product, or firm names in this publication is for descriptive purposes only and does not constitute endorsement by the U.S. Government.

For additional information

write to:

\section{District Chief}

U.S. Geological Survey

333 West Nye Lane, Room 203

Carson City, NV 89706-0866
Copies of this report may be purchased from:

U.S. Geological Survey

Earth Science Information Center

Open-File Reports Section

Box 25425, MS 517

Denver Federal Center

Denver, CO 80225-0046 


\section{CONTENTS}

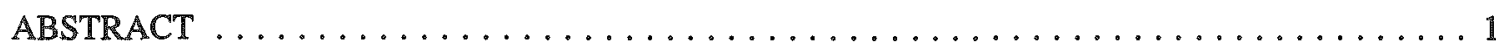

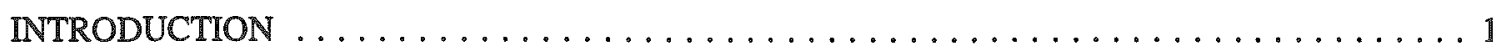

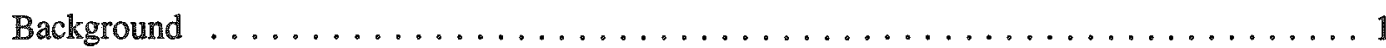

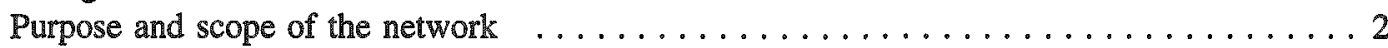

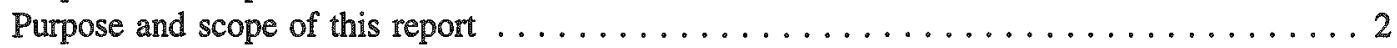

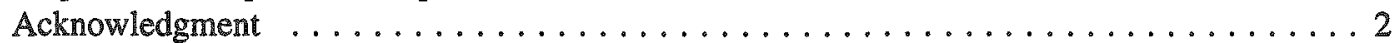

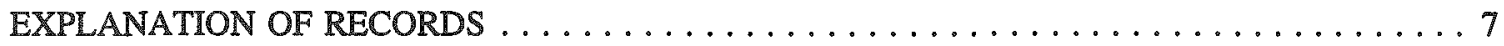

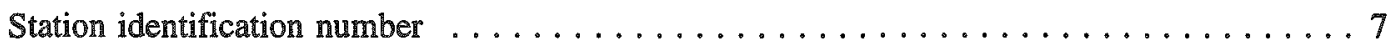

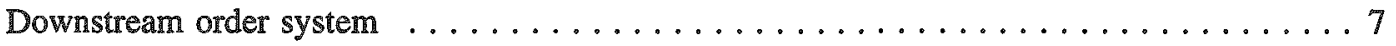

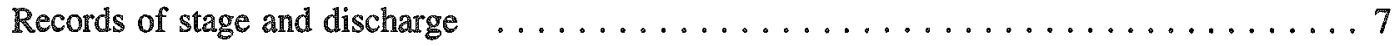

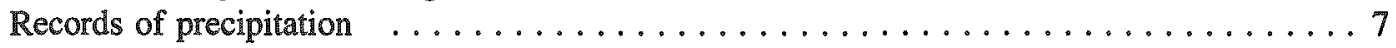

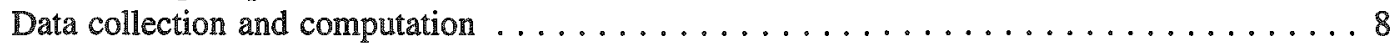

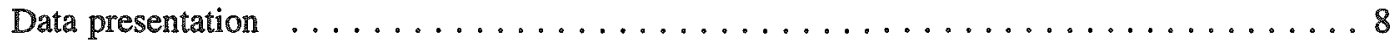

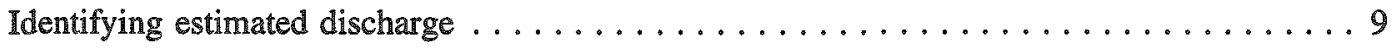

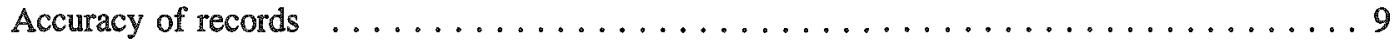

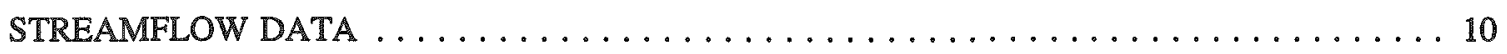

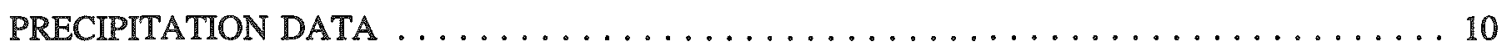

SUMMARY OF STREAMFLOW AND PRECIPITATION DATA DURING

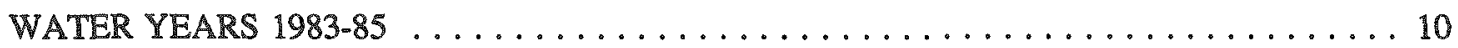

REFERENCES CITED . . . . . . . . . . . . . . . . . . . . . . . . 19

\section{ILLUSTRATIONS}

Figures 1-4. Maps for water years 1983-85 showing:

1. Location of U.S. Geological Survey regional streamflow stations ........ 3

2. Location of U.S. Geological Survey miscellaneous streamflow measurement sites $\ldots \ldots \ldots \ldots \ldots \ldots \ldots \ldots . \ldots 4$

3. Location of U.S. Geological Survey regional precipitation stations . . . . . . . 5

4. Location of Weather Service Nuclear Support Office precipitation stations . . . 6

5-6. Graphs showing streamflow at gages 10251248,10251250 ,

10251255, and 10251258 during:

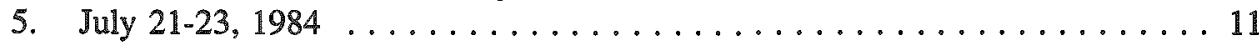

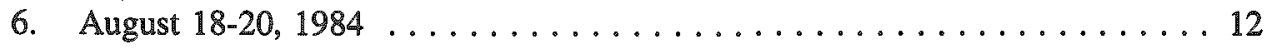

7-8. Maps showing cumulative measured precipitation at U.S. Geological Survey and

Weather Service Nuclear Support Office gages for:

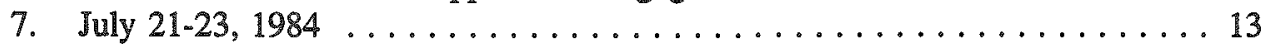

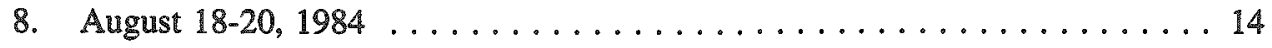




\section{TABLES}

Table 1. Maximum peak and maximum daily mean discharge for period of continuous record during water years $1983-85$ at complete-record stations $\ldots \ldots \ldots \ldots 10$

2. Discharge at U.S. Geological Survey complete-record stations . . . . . . . . . . 16

3. Discharge at U.S. Geological Survey crest-stage, partial-record stations . . . . . . 25

4. Discharge at U.S. Geological Survey miscellaneous sites . . . . . . . . . . 27

5. Precipitation data from U.S. Geological Survey network stations . . . . . . . . . 28

6. Weather Service Nuclear Support Office precipitation gaging stations . . . . . . . 30

7. Precipitation data from Weather Service Nuclear Support Office network stations . . 31

\section{CONVERSION FACTORS AND ABBREVIATIONS}

\begin{tabular}{lcl}
\multicolumn{1}{c}{ Multiply } & \multicolumn{1}{c}{ By } & \multicolumn{1}{c}{ To obtain } \\
acre-foot (acre-ft) & 1,233 & cubic meter \\
foot (ft) & 0.03048 & meter \\
cubic foot per second $\left(\mathrm{ft}^{3} / \mathrm{s}\right)$ & $0.02832 \times 10^{-2}$ & cubic meter per second \\
inch (in.) & & centimeter \\
mile (mi) & 25.4 & kilometer \\
square mile (mi & 1.609 & square kilometer \\
& 2.59 &
\end{tabular}

\section{SEA LEVEL}

In this report, "sea level" refers to the National Geodetic Vertical Datum of 1929 (NGVD of 1929, formerly called "Sea-Level" Datum of 1929), which is derived form a general adjustment of the first-order leveling networks of both the United States and Canada. 


\title{
STREAMFLOW AND SELECTED PRECIPITATION DATA FOR YUCCA MOUNTAIN AND VICINITY, NYE COUNTY, NEVADA, WATER YEARS 1983-85
}

\author{
By Marilyn E. Pabst, David A. Beck, \\ Patrick A. Glancy, and Jeffrey A. Johnson
}

\begin{abstract}
Streamflow and precipitation data collected at and near Yucca Mountain, Nevada, during water years 1983-85, are presented in this report. The data were collected and compiled as part of the studies the U.S. Geological Survey is making, in cooperation with the U.S. Department of Energy, to characterize surface-water hydrology in the Yucca Mountain area. Streamflow data include daily mean discharges and peak discharges at 4 complete-record gaging stations and peak discharges at 10 crest-stage, partial-record stations and 12 miscellaneous sites. Precipitation data include cumulative totals at 12 stations maintained by the U.S. Geological Survey and daily totals at 17 stations maintained by the Weather Service Nuclear Support Office, National Oceanic and Atmospheric Administration.
\end{abstract}

\section{INTRODUCTION}

\section{Background}

The Yucca Mountain area, on and adjacent to the Nevada Test Site (NTS) in Nye County, southern Nevada, is being investigated as a candidate site for the underground storage of high-level nuclear wastes. The investigation is a scientific site-characterization process that includes efforts to develop knowledge of the hydrogeology of Yucca Mountain and surrounding areas.

One component of the hydrogeologic investigation is characterization of streamflow at Yucca Mountain and throughout the region (fig. 1) to better understand relations between precipitation and runoff. A quantitative understanding of runoff is necessary to increase knowledge regarding ground-water recharge, flood- and debris-hazard potentials, and the relation between current and future hydrologic processes and those of the past.

Historical streamflow and precipitation data are nonexistent for the Yucca Mountain area and scarce for southern Nevada. Some peak-flow data have been collected by the U.S. Geological Survey (USGS) since the early 1960's in selected local and regional drainage areas (Moosburner, 1978). Flood hazards in the vicinity of Yucca Mountain have been described by Christensen and Spahr (1980) and Squires and Young (1984). Because historical data are scarce, a streamflow and precipitation network was established by the USGS, in cooperation with the U.S. Department of Energy. 


\section{Purpose and Scope of the Network}

The streamflow-data collection program began during the 1983 water year (October 1, 1982, to September 30,1983 ) as part of the Nevada Nuclear Waste Storage Investigation, now known as the Yucca Mountain Project (YMP). Concurrently, some precipitation data were collected to provide a reconnaissance perspective on the characteristics of precipitation related to runoff in the areas of stream-gaging networks.

The streamflow-measurement network is composed of three components: (1) A primary group of complete-record gaging stations (fig. 1), (2) a secondary group of crest-stage, partial-record gaging stations with peak-stage recording devices (fig. 1), and (3) a tertiary group of miscellaneous sites selected for quantitative determination of peak streamflow during specific runoff events (fig. 2). Both primary and secondary stations are visited periodically for routine inspection and maintenance of stage recording equipment and to determine if streamflow has occurred. If streamflow occurred, measurements of the peak streamflow rates are made according to standard USGS techniques (Benson and Dalrymple, 1967; Dalrymple and Benson, 1967; Hulsing, 1967; Bodhaine, 1968).

Similar measurements are made at tertiary sites, where no gages are located. Tertiary-measurement sites are selected for given runoff events on the basis of the hydrologic characteristics of the specific event. The tertiary sites are not revisited periodically or measured repetitively, as are the primary and secondary stations. Tertiary sites can be remeasured if additional data are needed.

Precipitation-measurement gages are operated at most of the primary and secondary stream-gaging stations. A few precipitation gages are located where additional data are needed (fig. 3). The precipitationmeasurement network was not designed to provide comprehensive coverage of meteorological events in the area, but to provide supplementary information about precipitation, with an emphasis on cost effectiveness and logistical compatibility with the streamflow-measurement network. The data provide additional quantitative perspective on the amount of precipitation associated with runoff for drainages that have stream gages.

The Weather Service Nuclear Support Office (WSNSO), National Oceanic and Atmospheric Administration, designed and has operated a precipitation-measurement network at NTS (fig. 4) since the early 1960's (Quiring, 1983). The resultant data base provides the basic knowledge of precipitation at the NTS area for almost the last quarter century. Although the network was not designed to define precipitation-runoff relations, precipitation data obtained enhance the understanding of precipitation-runoff relations in the Yucca Mountain area.

\section{Purpose and Scope of this Report}

The purpose of this report is to document streamflow and precipitation data collected by USGS during water years 1983-85 for the streamflow-studies component of YMP hydrogeologic studies. The report also includes selected precipitation data collected by WSNSO through their NTS precipitation network. WSNSO data are included in this report because these data can be used to relate storm activity and its potential for runoff in the area and the region.

\section{Acknowledgment}

Special thanks is given to Charles Steadman, Weather Service Nuclear Support Office, for his assistance in compiling WSNSO precipitation data. 


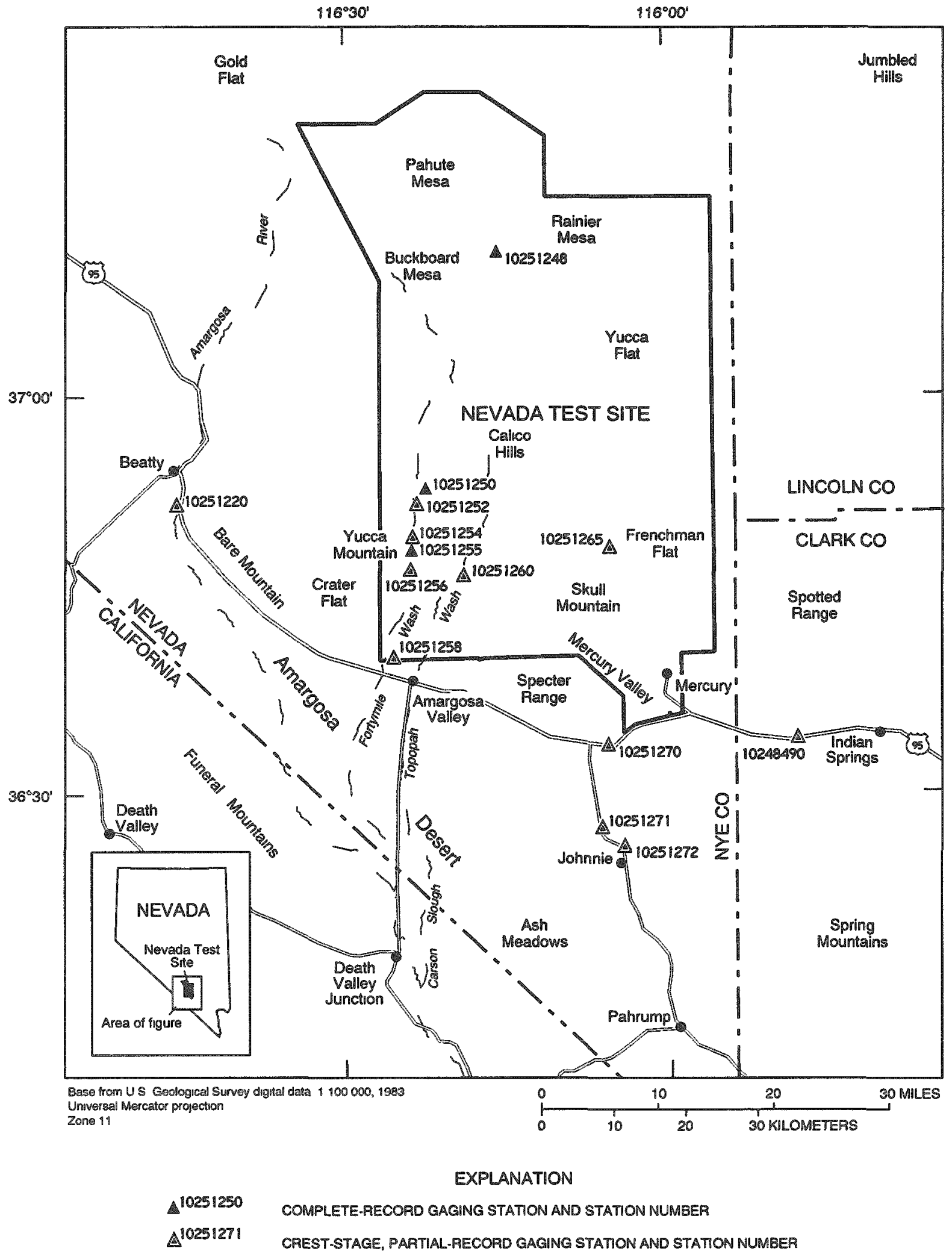

FIGURE 1.--Location of U.S. Geological Survey regional streamflow stations for water years $1983-85$. 


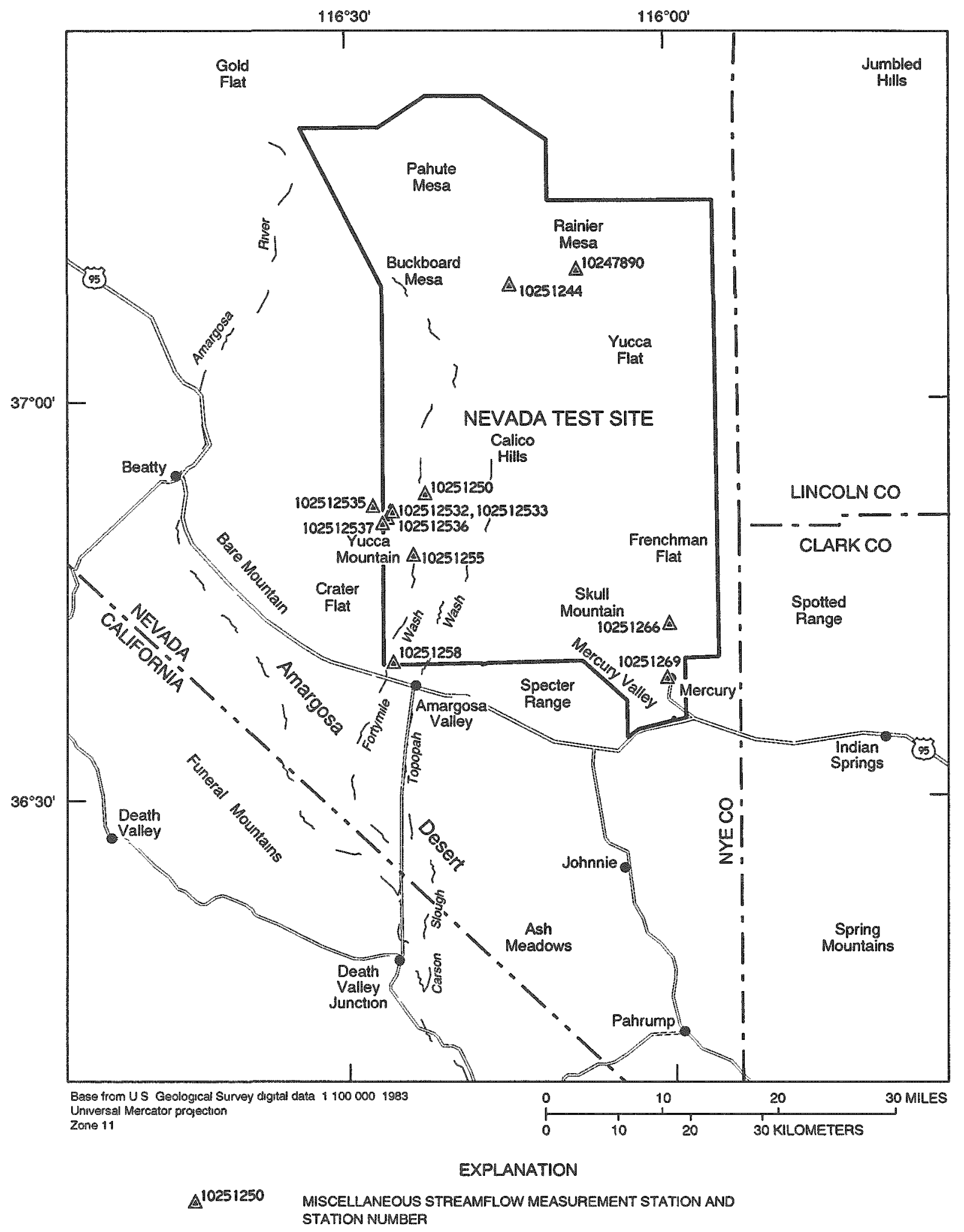

FIGURE 2.--Location of U.S. Geological Survey miscellaneous streamflow measurement sites for water years 1983-85. 


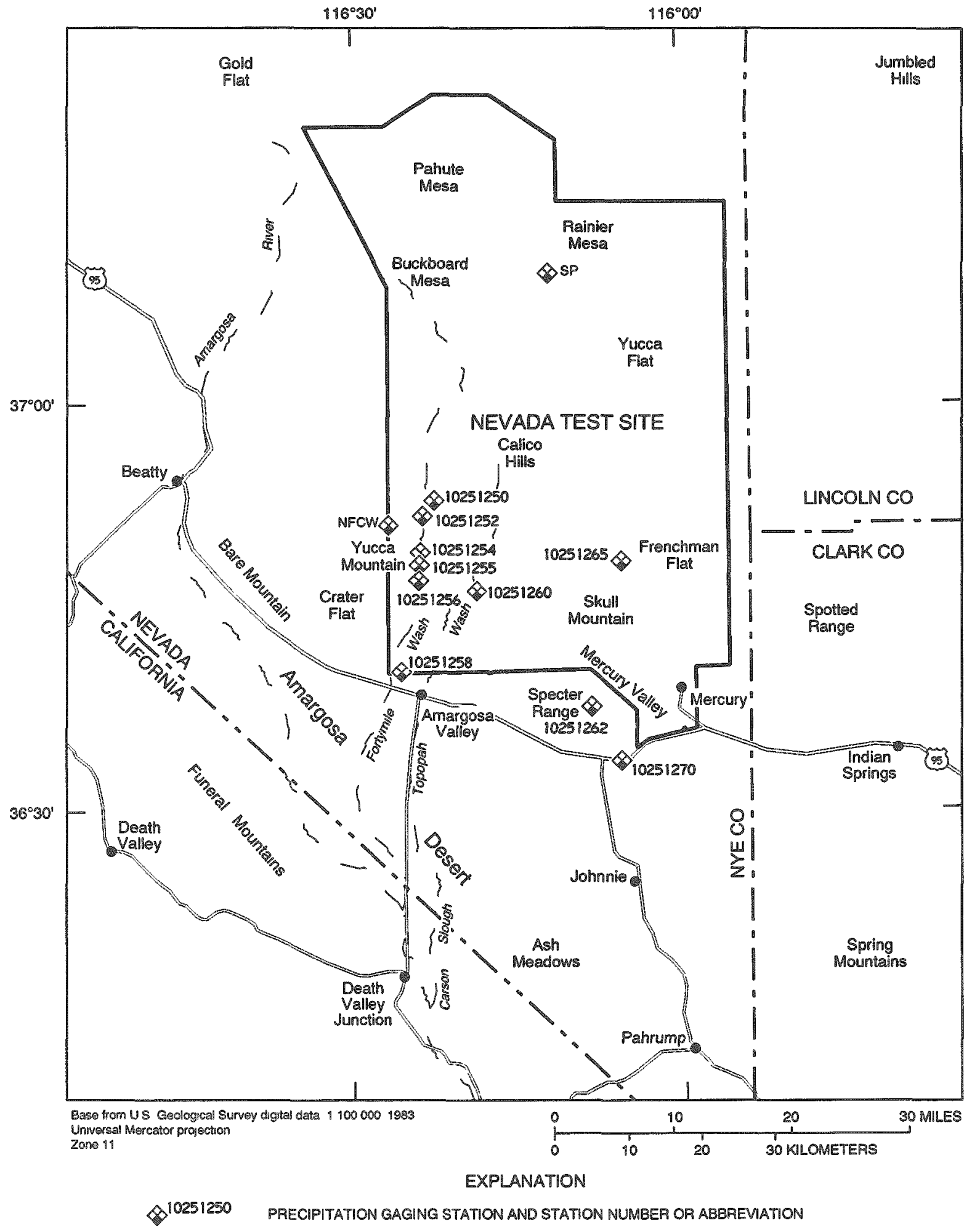

FIGURE 3.--Location of U.S. Geological Survey regional precipitation stations for water years 1983-85. 


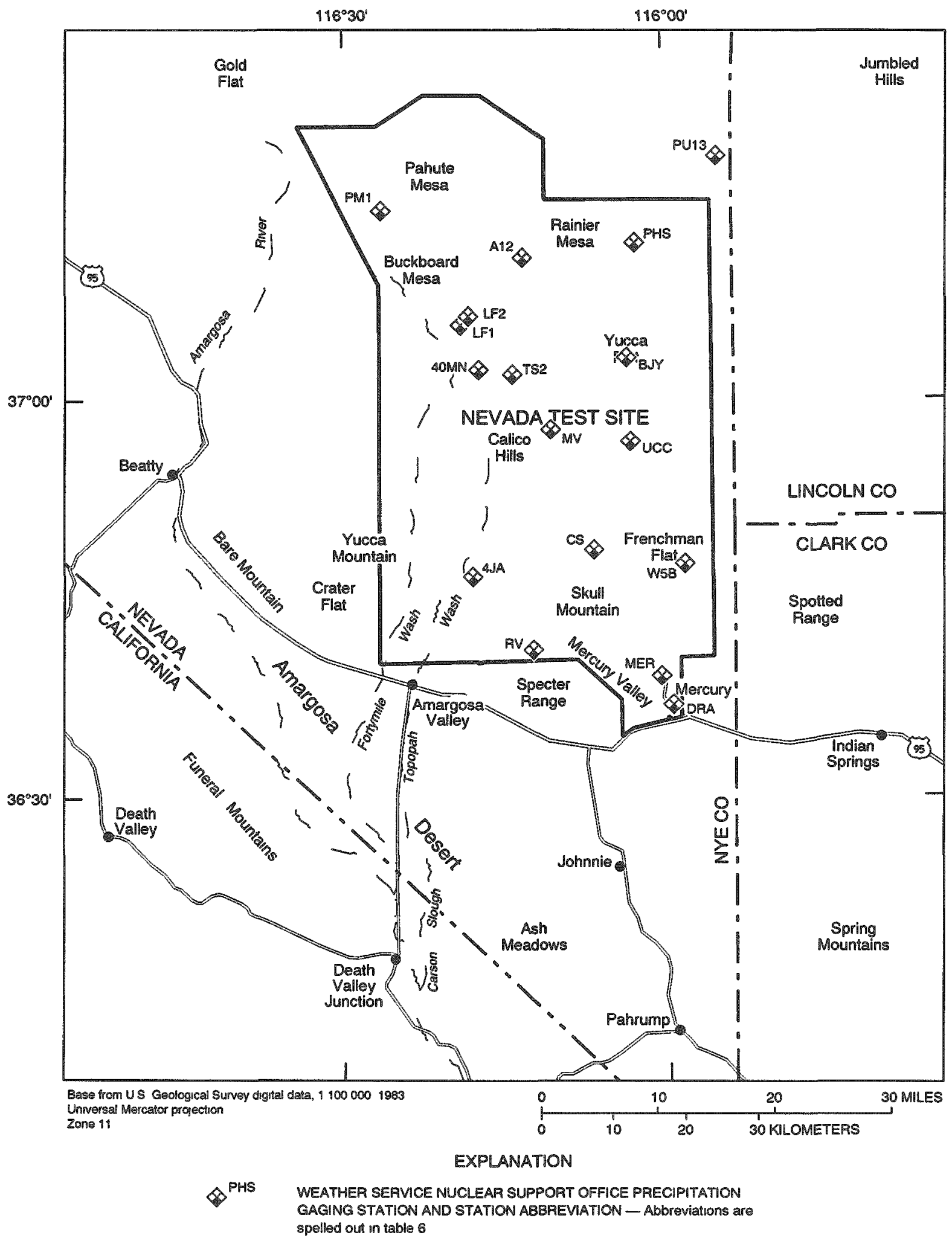

FIGURE 4.--Location of Weather Service Nuclear Support Office precipitation stations for water years 1983-85. 


\section{EXPLANATION OF RECORDS}

The surface-water and precipitation records included in this report are for water years 1983-85, which began October 1, 1982, and ended September 30, 1985. The following sections of text provide a more detailed explanation of how the hydrologic data in this report were collected, analyzed, and arranged for presentation.

\section{Station Identification Number}

Each surface-water data station in this report is assigned a unique identification number. This number is unique in that it applies specifically to a given station and to no other. The number usually is assigned when a station first is established and it is retained for that station indefinitely. The system used by USGS to assign identification numbers for surface-water stations is based on geographic location in downstream order. This downstream-order system is used for all surface-water stations in this report.

Local station abbreviations in this report identify WSNSO precipitation-measurement stations and USGS precipitation-measurement stations. The station abbreviations are derived from the name of the site and may consist of letters, numbers, or both.

\section{Downstream Order System}

Since October 1, 1950, the order of listing hydrologic-station records in Survey reports has been in a downstream direction along the main stream. All stations on a tributary entering from a main-stream station are listed before that station. A station on a tributary that enters between two main-stream stations is listed between them.

Downstream order along a stream is represented by the sequential numbering of stations. In assigning the station numbers, no distinction is made between partial-record stations and complete-record stations; therefore, the station number for a partial-record station indicates downstream-order position in a list made up of both types of stations. Gaps are left in the series of numbers to allow for new stations that may be established; hence the numbers are not consecutive. For example, the complete 8-digit number for station 10251255 includes the 2-digit part number 10 , plus the 6-digit downstream-order number 251255 . The part number refers to an area the boundaries of which coincide with certain natural drainage lines. Records in this report are for sites in Part 10, the Great Basin. When a station is added between two consecutively numbered stations, an additional digit is added to the upstream station number.

\section{Records of Stage and Discharge}

Records of stage and discharge may be complete or partial. Complete records of discharge are those obtained using a continuous stage-recording device through which either instantaneous or daily mean discharges may be computed for any time during the period of record. In contrast, partial records are obtained through discrete measurements without using a continuous stage-recording device and pertain to only a few flow characteristics.

\section{Records of Precipitation}

USGS operates storage rain gages at complete-record stations, partial-record stations, and unique precipitation stations. These precipitation stations are designed to supplement existing and planned precipitation networks within the study area.

WSNSO operates a precipitation network designed to give broad areal coverage of precipitation within the study area. Records of precipitation are obtained from storage, tipping-bucket, or weighing rain gages. Some stations have more than one type of rain gage to ensure continuous records. 


\section{Data Collection and Computation}

The data obtained at a complete-record gaging station on a stream consist of a continuous record of stage, individual measurements of discharge, and notations regarding factors that may affect the relation between stage and discharge. These data, together with supplemental information, such as weather records, are used to compute daily discharges (Buchanan and Somers, 1968, 1969; Carter and Jacob, 1968; Kennedy, 1983, 1984).

Continuous records of stage are obtained with analog recorders that trace continuous levels of stage. Measurements of peak discharge are made indirectly using methods adopted by the Geological Survey as a result of experience since 1880 (Benson and Dalrymple, 1967; Dalrymple and Benson, 1967; Hulsing, 1967; Bodhaine, 1968).

\section{Data Presentation}

The records published for each complete-record gaging station consist of two parts, the manuscript and the data table, for each of 3 water years (1983-85) that the gage was in operation. The manuscript provides information under several headings, including station location, period of record, historical extremes, record accuracy, and other remarks pertinent to station operation and regulation. The following information, as appropriate, is provided for each continuous record of discharge.

LOCATION.--Information on location is obtained from the most accurate map available. Location of the gage is given with respect to cultural and physical features in the vicinity and with respect to the reference place mentioned in the station name.

DRAINAGE AREA.--Drainage area is measured using the most accurate maps available. Because the types of maps available differ from one drainage basin to another, the accuracy of drainage areas differs. Drainage areas are updated and revised as better maps become available.

REVISED RECORDS.--Published material has been revised. The previously published report is indicated, as is the changed information.

PERIOD OF RECORD.--This indicates the period for which records for the station or for an equivalent station have been published. An equivalent station is one that was in operation when the present station was not and whose location was such that records from it can reasonably be considered equivalent to records from the present station.

GAGE.--The type of gage in current use, the elevation of the gage and how it was determined, and a condensed history of the types, locations, and datums of previous gages may be given.

REMARKS.--This paragraph is used to present information relative to the accuracy of records, to special methods of computation, to conditions that affect natural flow at the station, and to other pertinent items as necessary.

EXTREMES FOR PERIOD OF RECORD.--Extremes may include maximum and minimum stages and maximum and minimum discharges. Unless otherwise qualified, maximum discharge is the instantaneous maximum corresponding to the highest stage that occurred. The highest stage may have been obtained from a graphic recorder, a crest-stage gage, or by direct observation of a nonrecording gage. If the maximum stage did not occur on the same day as the maximum discharge, it is given separately. Similarly, the minimum is the instantaneous minimum discharge, unless otherwise qualified, and was determined and is reported in the same manner as the maximum.

EXTREMES OUTSIDE PERIOD OF RECORD.--Extremes, such as major floods, that occurred outside the stated period of record are reported. 
EXTREMES FOR CURRENT YEAR.--Extremes similar to those for the period of record, except the peak discharge is the maximum for the water year, are reported.

Data for complete-record gaging stations (table 2) consist of mean discharge for each day followed by monthly and yearly summaries. In the monthly summary below the table, "TOTAL" is the sum of daily values. "MEAN" is the average flow in cubic feet per second during the month. "MAX" and "MIN" are the maximum and minimum daily discharges, respectively, for the month. "AC-FT" is the monthly discharge in acre-feet. Below this, "WTR YR" summarizes discharge for the water year.

Data for partial-record discharge stations (table 3) include location, drainage area, period of record, dates of measurements, stage (when applicable), and discharge for all measurements made at crest-stage stations during the reporting period. Table 4 contains discharge measurements made during the reporting period at sites other than complete-record or partial-record stations. These measurements generally are made in times of flood to give additional areal coverage. Measurements at miscellaneous sites include location, drainage area, date of measurement, and discharge.

USGS precipitation data are totals for the specified period. The interval is the time between gage inspections. The data from WSNSO precipitation sites are presented in monthly tables with daily and monthly totals for each site.

\section{Identifying Estimated Discharge}

Estimated discharge values are identified by the letter " $e$ " preceding the value and by the headnote or footnote "e Estimated."

Discharge values are estimated by one or more of the following methods: slope-conveyance computation, hydrographic comparison with nearby stations or local weather records, extrapolation of hydrographs based on recessional characteristics, or visual estimates of channel characteristics or hydraulics.

\section{Accuracy of Records}

The accuracy of streamflow records depends primarily on (1) stability of the stage-discharge relation or, if the control is unstable, frequency of discharge measurements, and (2) accuracy of measurements of stage, measurements of discharge, and interpretation of records.

The accuracy attributed to the records is indicated under "REMARKS." "Excellent" means about 95 percent of the daily discharges are within 5 percent of their true values; "good," within 10 percent; and "fair," within 15 percent. Records that do not meet the criteria mentioned are rated "poor." Different accuracies may be attributed to different parts of a given record.

Daily mean discharges in this report are given to the nearest $0.01 \mathrm{ft}^{3} / \mathrm{s}$ for values less than $1 \mathrm{ft}^{3} / \mathrm{s}$; to the nearest $0.1 \mathrm{ft}^{3} / \mathrm{s}$ between $1.0 \mathrm{ft}^{3} / \mathrm{s}$ and $10 \mathrm{ft}^{3} / \mathrm{s}$; to whole numbers between $10 \mathrm{ft}^{3} / \mathrm{s}$ and $1,000 \mathrm{ft}^{3} / \mathrm{s}$; and to three significant figures for more than $1,000 \mathrm{ft}^{3} / \mathrm{s}$. The number of significant figures reported is based solely on the magnitude of the discharge value. The same rounding applies to discharges for partialrecord stations and miscellaneous sites.

The precipitation values for USGS sites are given to the nearest 0.1 in. The precipitation values for WSNSO sites are given to the nearest $0.01 \mathrm{in}$. 


\section{STREAMILOW DATA}

Records of streamflow data collected during water years 1983-85 at four USGS complete-record gaging stations (primary sites) are listed in table 2. Records of peak streamflow data collected during the same period at 10 USGS crest-stage, partial-record stations (secondary sites) are listed in table 3. Also, records of streamflow data collected during the reporting period at 12 miscellaneous (tertiary) sites are listed in table 4.

\section{PRECIPITATION DATA}

Records of precipitation data collected during water years 1983-85 at 12 USGS stations, and information regarding the stations themselves, are listed in table 5. Information regarding the WSNSO stations is listed in table 6 , and records of precipitation are listed in table 7.

\section{SUMMARY OF STREAMFLOW AND PRECIPITATION DATA DURING WATER YEARS 1983-85}

Records of streamflow were collected and compiled during water years 1983-85 from 4 completerecord gaging stations, 10 crest-stage, partial-record stations, and 12 miscellaneous sites. A summary of maximum peak and maximum daily mean discharges during water years 1983-85 for the complete-record gaging stations is given in table 1. Streamflow hydrographs for the four complete-record stations during July 21-23, 1984, and August 18-20,1984, are shown in figures 5 and 6 . These two periods include the only occurrences of appreciable flow during the overall period of continuous record at the four stations. At the crest-stage, partial-record stations, the maximum peak discharge measured was $1,150 \mathrm{ft}^{3} / \mathrm{s}$ on August 19, 1983, at Amargosa River Tributary near Mercury (10251270). At the miscellaneous sites, the maximum peak discharge measured was $1,520 \mathrm{ft}^{3} / \mathrm{s}$ on March 3, 1983, at Fortymile Wash at Narrows (10251250).

Precipitation data also were collected and compiled for water years 1983-85 for 12 USGS precipitation stations and 17 WSNSO precipitation stations. USGS precipitation network consisted of storage rain gages that were measured periodically; these gages were intended to supplement existing and future precipitation networks on or near NTS. Cumulative precipitation measured during the periods July 21-23, 1984, and August 18-20, 1984, for USGS and selected WSNSO network gages are shown in figures 7 and 8 , respectively.

TABLE 1.--Maximum peak and maximum daily mean discharge for period of continuous record during water years 1983.85 at complete-record stations

\begin{tabular}{|c|c|c|c|c|c|c|}
\hline \multirow[b]{2}{*}{$\begin{array}{l}\text { Station } \\
\text { number }\end{array}$} & \multirow[b]{2}{*}{ Station name } & \multirow[b]{2}{*}{$\begin{array}{l}\text { Period of } \\
\text { continuous record }\end{array}$} & \multicolumn{2}{|c|}{ Maxinum peak discharge } & \multicolumn{2}{|c|}{$\begin{array}{l}\text { Maximum daily mean } \\
\text { discharge (estimated) }\end{array}$} \\
\hline & & & $\begin{array}{l}\text { Cubic feet } \\
\text { per second }\end{array}$ & Date & $\begin{array}{l}\text { Cubic feet } \\
\text { per second }\end{array}$ & Date \\
\hline 10251248 & $\begin{array}{l}\text { Unnamed Tributary to } \\
\text { Fortymile Wash near } \\
\text { Rattlesnake Ridge }\end{array}$ & $05-23-84$ to $09-30-85$ & $10 \mathrm{e}$ & $07-21-84$ & 0.26 & $08-19-84$ \\
\hline 10251250 & $\begin{array}{l}\text { Fortymile Wash } \\
\text { at Narrows }\end{array}$ & $09-21-83$ to $09-30-85$ & 730 & $07-21-84$ & 56 & $07-21.84$ \\
\hline 10251255 & $\begin{array}{r}\text { Fortymile Wash } \\
\text { near Well J-13 }\end{array}$ & $11-30-83$ to $09-30-85$ & 1,860 & $07.21-84$ & 60 & $07-21.84$ \\
\hline 10281258 & $\begin{array}{c}\text { Fortymile Wash near } \\
\text { Amargosa Valley }\end{array}$ & $11-15-83$ to $09-30-85$ & 1,430 & $07-22-84$ & 95 & $07-22-84$ \\
\hline
\end{tabular}

e Estimated. 

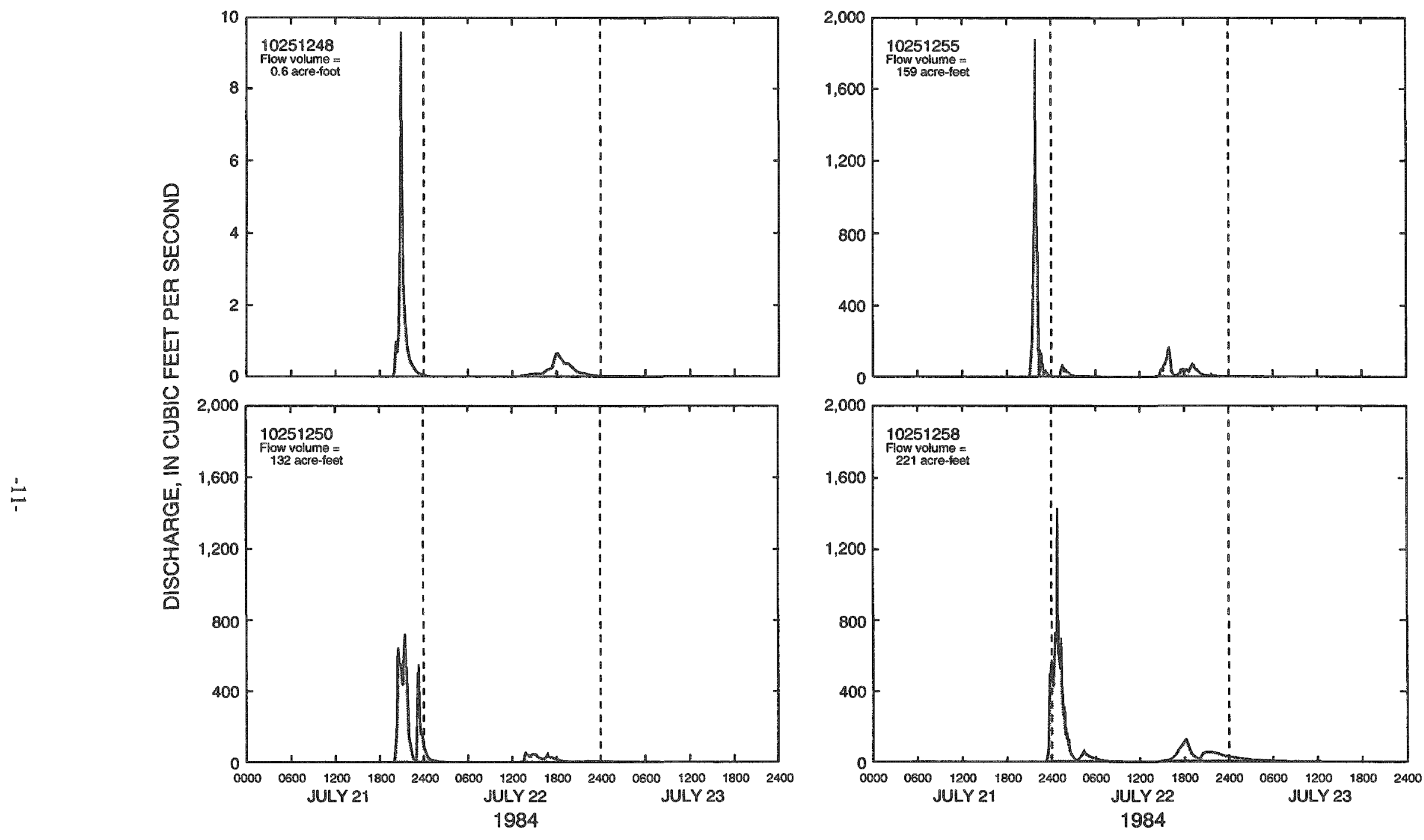

FIGURE 5.--Streamflow at gages $10251248,10251250,10251255$, and 10251258 during July 21-23, 1984. 

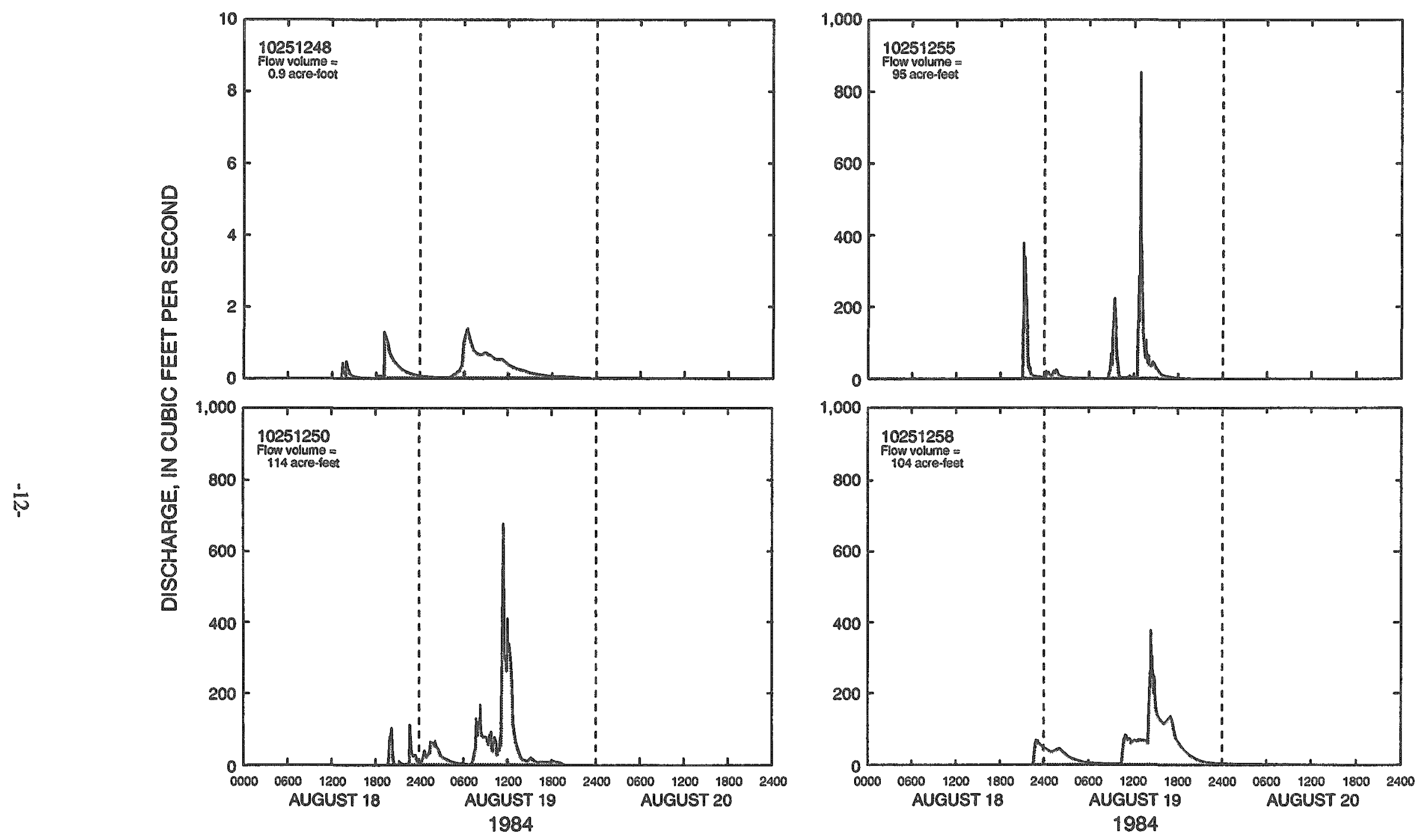

FIGURE 6.--Streamflow at gages 10251248, 10251250, 10251255, and 10251258 during August 18-20, 1984 . 


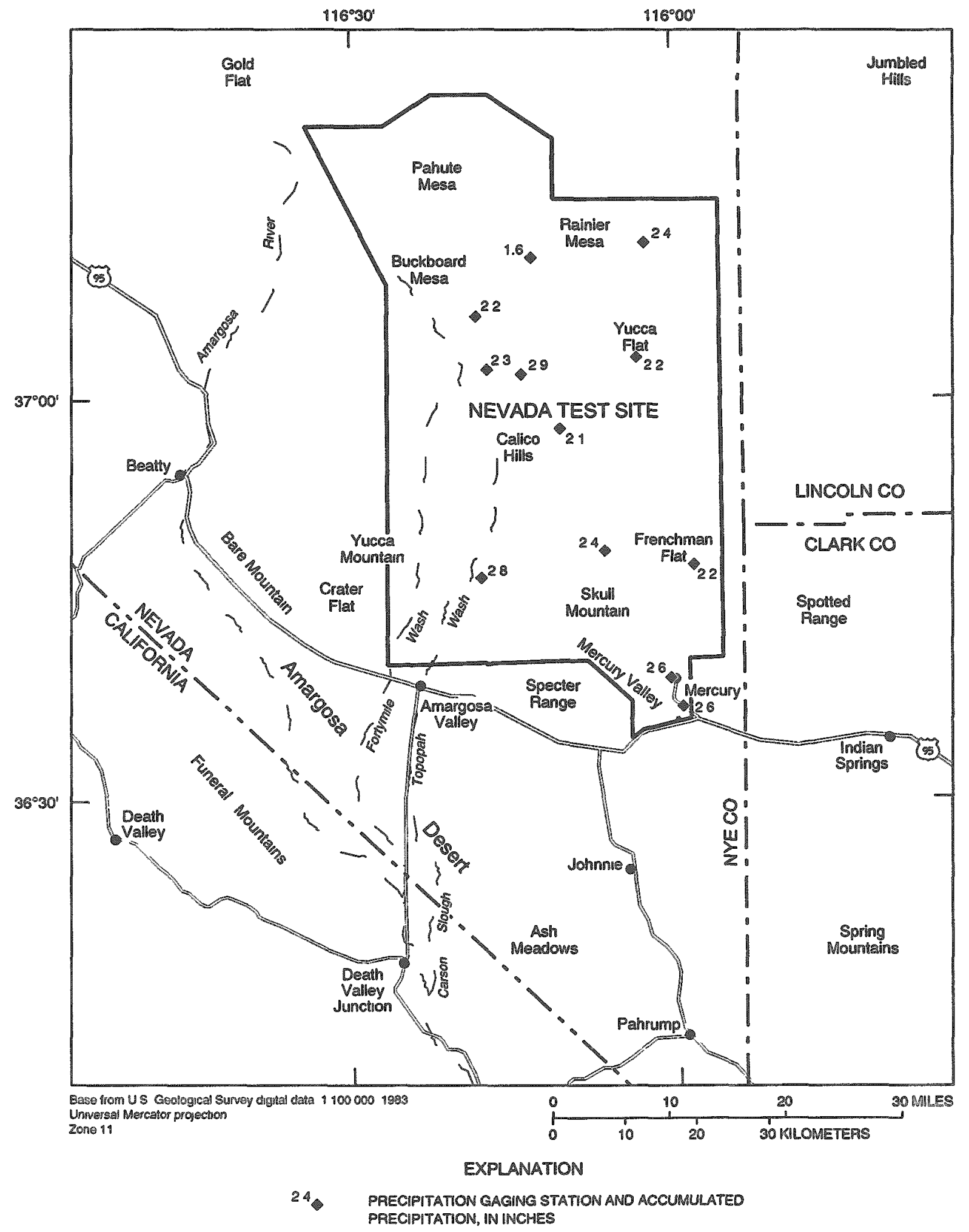

FIGURE 7.--Cumulative measured precipitation at U.S. Geological Survey and Weather Service Nuclear Support Office gages for July 21-23, 1984. 


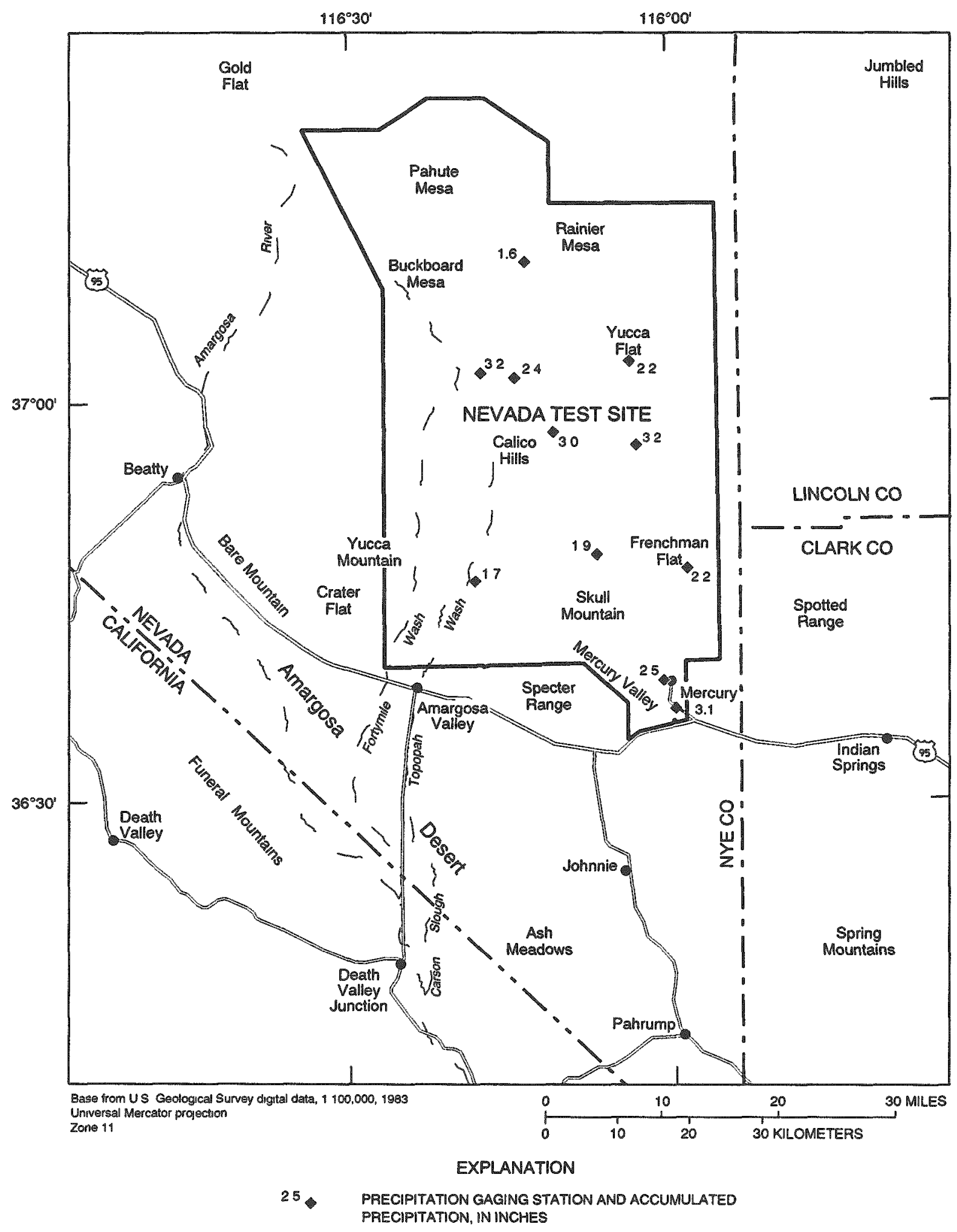

FIGURE 8.--Cumulative measured precipitation at U.S. Geological Survey and

Weather Service Nuclear Support Office gages for August 18-20, 1984. 


\section{RENERENCES CITED}

Benson, M.A., and Dalrymple, Tate, 1967, General field and office procedures for indirect discharge measurements: U.S. Geological Survey Techniques of Water-Resources Investigations, Book 3, Chapter $\mathrm{A} 1,30 \mathrm{p}$.

Bodhaine, G.L., 1968, Measurement of peak discharge at culverts by indirect methods: U.S. Geological Survey Techniques of Water-Resources Investigations, Book 3, Chapter A3, 60 p.

Buchanan, T.J., and Somers, W.P., 1968, Stage measurements at gaging stations: U.S. Geological Survey Techniques of Water-Resources Investigations, Book 3, Chapter A7, 28 p.

-----1969, Discharge measurements at gaging stations: U.S. Geological Survey Techniques of WaterResources Investigations, Book 3, Chapter A8, 65 p.

Carter, R.W., and Davidian, Jacob, 1968, General procedure for gaging streams: U.S. Geological Survey Techniques of Water-Resources Investigations, Book 3, Chapter A6, 13 p.

Christensen, R.C., and Spahr, N.E., 1980, Flood potential of Topopah Wash and tributaries, eastern part of Jackass Flats, Nevada Test Site, southern Nevada: U.S. Geological Survey Open-File Report 80$963,26 \mathrm{p}$.

Dalrymple, Tate, and Benson, M.A., 1967, Measurement of peak discharge by the slope-area method: U.S. Geological Survey Techniques of Water-Resources Investigations, Book 3, Chapter A2, 12 p.

Hulsing, Harry, 1967, Measurement of peak discharge at dams by indirect methods: U.S. Geological Survey Techniques of Water-Resources Investigations, Book 3, Chapter A5, 29 p.

Kennedy, E.J., 1983, Computation of continuous records of streamflow: U.S. Geological Survey Techniques of Water-Resources Investigations, Book 3, Chapter A13, 53 p.

---1984, Discharge ratings at gaging stations: U.S. Geological Survey Techniques of Water-Resources Investigations, Book 3, Chapter A10, 59 p.

Moosburner, Otto, 1978, Flood investigations in Nevada through 1977 water year--progress report 17: U.S. Geological Survey Open-File Report 78-610, 90 p.

Quiring, R.F., 1983, Precipitation climatology of the Nevada Test Site: U.S. Department of Commerce, National Weather Service, $34 \mathrm{p}$.

Squires, R.R., and Young, R.L., 1984, Flood potential of Fortymile Wash and its principal southwestern tributaries, Nevada Test Site, southern Nevada: U.S. Geological Survey Water-Resources Investigations Report 83-4001, 33 p.

U.S. Geological Survey, 1964-65, Surface water records of Nevada, 1963-64: Carson City. Nev., U.S. Geological Survey water-data reports (published annually).

---1966-75. Water resources data for Nevada, water years 1965-74: Carson City, Nev., U.S. Geological Survey water-data reports (published annually).

--n-1976-85, Water resources data, Nevada, water years 1975-85: U.S. Geological Survey Water-Data Reports NV-75-1 to NV-85-1 (published annually). 
[AC-FT, acre-feet; e. estimated; $\mathrm{h}$, feet; $\mathrm{ft}^{3} / \mathrm{s}$, cubic feet per second; lat, latitude; long, longitude; MAX, maximum; mi, mile; mi $^{2}$, square mile; MN, minimum; WTR YR, water year]

\section{AMARGOSA DESERT BASIN}

\section{UNMAMED TRIBUTARY TO STOCKADE WASH NEAR RATTLESNARE RIDGE, NEVADA TEST STTE, NEVADA}

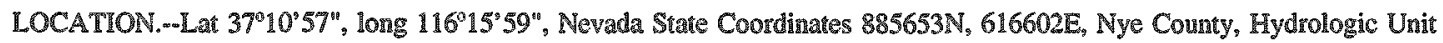
18090202, on Nevada Test Site, on right bank 200 ft upstream from Pahute Mesa Road, and 39 mi north-norhwest of Mercury.

DRAINAGE AREA.--3.90 $\mathrm{mi}^{2}$.

PERIOD OF RECORD.-MAy 23, 1984 , to curent year.

GAGE.--Water-stage recorder. Elevation of gage is 5,980 fi above sea level, from topographic map.

REMARKS.--Reconds poor.

EXTREMES FOR PERIOD OF RECORD.--Maximum discharge, $10 \mathrm{fl}^{3} / \mathrm{s}$ (estimated), July 21,1984 , on basis of slope-conveyance measurement of peak flow, gage height, 4.60 ft; no flow most days, most years.

EXTREMES FOR CURRENT PERIOD.-May to September 1984: Maximum discharge during period, $10 \mathrm{fi}^{3} / \mathrm{s}$ (estimated), July 21 , on basis of slope-conveyance measurement of peal flow, gage height, 4.60 ft; no llow most days.

\begin{tabular}{|c|c|c|c|c|c|c|c|c|c|c|c|c|}
\hline \multirow[b]{2}{*}{ DAY } & \multicolumn{11}{|c|}{$\begin{array}{l}\text { DISCHARGE, IN CUBIC FEET PER SECOND, WATER YEAR OCTOBER } 1983 \text { TO SEPTEMBER } 1984 \\
\text { DAILY MEAN VALUES }\end{array}$} & \multirow[b]{2}{*}{ SEP } \\
\hline & OCT & NOV & DEC & IAN & FEB & MAR & APR & $\mathrm{MAY}$ & JUN & JUL & AUG & \\
\hline 1 & - & -- & $=$ & - & $-\infty$ & $=$ & - & $=$ & 0.00 & 0.00 & 0.00 & 0.00 \\
\hline 2 & $\ldots$ & $=$ & - & - & $\propto-$ & - & - & -- & .00 & .00 & .00 & .00 \\
\hline 3 & $\infty$ & $=$ & -. & $-\infty$ & - & $\ldots$ & $\ldots$ & $=$ & .00 & .00 & .00 & .00 \\
\hline 4 & $-\infty$ & -- & - & -- & $\ldots$ & - & - & $=$ & .00 & .00 & .00 & .00 \\
\hline 5 & - & - & - & -- & - & $=$ & - & - & .00 & .00 & .00 & .00 \\
\hline 6 & -- & $\ldots$ & - & 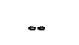 & - & $=$ & $\ldots$ & $=$ & .00 & .00 & .00 & .00 \\
\hline 7 & -- & 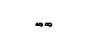 & -- & $\ldots$ & $=$ & - & $\ldots$ & - & .00 & .00 & .00 & .00 \\
\hline 8 & $\ldots$ & -- & -. & $=$ & - & $\infty$ & -- & - & .00 & .00 & .00 & .00 \\
\hline 9 & -. & - & - & - & - & - & -- & - & .00 & .00 & .00 & .00 \\
\hline 10 & - & - & -. & -. & - & -. & $\ldots$ & - & .00 & .00 & .00 & .00 \\
\hline 11 & $\ldots$ & -- & $\ldots$ & $-\infty$ & $\infty$ & - & $\approx$ & $\ldots$ & .00 & .00 & .00 & .00 \\
\hline 12 & .. & - & - & -. & $\ldots$ & $=$ & $\ldots$ & $\ldots$ & .00 & .00 & .00 & .00 \\
\hline 13 & $\ldots$ & -. & $=$ & $\ldots$ & $\infty$ & - & -. & $=$ & .00 & .00 & .00 & .00 \\
\hline 14 & -- & -- & -. & $\ldots$ & -. & $\ldots$ & -- & - & .00 & .00 & .00 & .00 \\
\hline 15 & $=$ & -. & -- & -- & -- & - & -- & - & .00 & .00 & $e .01$ & .00 \\
\hline 16 & $\infty$ & -. & $-\infty$ & $\ldots$ & $\infty$ & $\ldots$ & - & $=$ & .00 & .00 & .00 & .00 \\
\hline 17 & -- & -. & -. & $-\infty$ & $\ldots$ & $\infty$ & $\ldots$ & $\ldots$ & .00 & .00 & .00 & .00 \\
\hline 18 & $\ldots$ & $\infty$ & - & - & - & - & -- & - & .00 & .00 & .10 & .00 \\
\hline 19 & $\infty$ & -- & - & $\infty$ & - & $-\infty$ & -- & $\infty$ & .00 & .00 & e.26 & .00 \\
\hline 20 & -- & -- & -- & -. & -- & - & $\infty$ & - & .00 & .00 & $e .08$ & .00 \\
\hline 21 & $=$ & $=$ & - & - & $-\infty$ & -- & - & $\alpha$ & .00 & e.22 & .00 & .00 \\
\hline 22 & - & -- & - & - & - & $\ldots$ & $\infty$ & sm & .00 & 8.09 & .00 & .00 \\
\hline 23 & -- & -- & $=-$ & $\ldots$ & -- & -- & -- & 0.00 & .00 & .00 & .00 & .00 \\
\hline 24 & -- & $=$ & $\infty$ & - & $=$ & $\ldots$ & - & .00 & .00 & .00 & .00 & .00 \\
\hline 25 & -- & $\cdots$ & - & - & $\approx$ & - & - & .00 & .00 & .00 & .00 & .00 \\
\hline 26 & $\infty$ & - & -. & - & $\ldots$ & -- & - & .00 & .00 & .00 & .00 & .00 \\
\hline 27 & - & -- & - & - & $-\infty$ & - & $=$ & .00 & .00 & .00 & .00 & .00 \\
\hline 28 & -- & $=$ & $=$ & -. & -- & $\ldots$ & -- & .00 & .00 & .00 & .00 & .00 \\
\hline 29 & $=$ & $=$ & - & $\ldots$ & $=$ & $\ldots$ & - & .00 & .00 & .00 & .00 & .00 \\
\hline 30 & - & - & -- & $-\infty$ & -- & - & - & .00 & .00 & .00 & .00 & .00 \\
\hline 31 & - & - & $\ldots$ & - & - & $m$ & -- & .00 & -- & .00 & .00 & $\cdots$ \\
\hline TOTAL & - & $\ldots$ & -. & $=$ & -- & $=$ & - & -- & 0.00 & 0.31 & 0.45 & 0.00 \\
\hline MEAN & - & - & -- & - & - & -- & - & -- & .000 & .010 & .015 & .000 \\
\hline MAX & -- & $\infty$ & $\cdots$ & .. & $\ldots$ & $\ldots$ & - & - & .00 & .22 & .26 & .00 \\
\hline MIN & -- & -- & $\ldots$ & - & $\ldots$ & 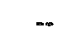 & $=$ & -- & .00 & .00 & .00 & .00 \\
\hline$A C \cdot F T$ & $=$ & - & -- & - & -- & -. & - & - & .00 & .6 & .9 & .00 \\
\hline
\end{tabular}




\section{AMARGOSA DESERT BASIN}

\section{UNNAMED TREUTARY TO STOCKADE WASH NEAR RATTLESNAKE RIDGE, NEVADA TEST SITE, NEVADA}

LOCATION.-Lat 37 $10^{\prime} 57^{\prime \prime}$, long $116^{\circ} 15^{\circ} 59^{\circ}$, Nevada State Coordinates 885653N, 616602E, Nye County, Hydrologic Unit 18090202 , on Nevada Test Site, on right bank 200 ft upstrearn from Pahute Mesa Road, and 39 mi north-northwest of Mercury.

DRAINAGE AREA.--3.90 $\mathrm{mi}^{2}$.

PERIOD OF RECORD.--May 23, 1984, to current year.

GAGE.--Water-stage recorder. Elevation of gage is 5,980 ft above sea level, from topographic map.

REMARKS.--Records poor.

EXTREMES FOR PERIOD OF RECORD.-Maximum discharge, $10 \mathrm{ft}^{3} / \mathrm{s}$ (estimated), July 21,1984 , on basis of slope-conveyance measurement of peak flow, gage height, $4.60 \mathrm{ft}$; no flow most days, most years.

EXTREMES FOR CURRENT PERIOD.-Water year 1985: Maximum discharge, $0.55 \mathrm{ft}^{3} / \mathrm{s}$ (estimated), October 2, gage height, 3.94 ft; no flow most days.

DISCHARGE, IN CUBIC FEET PER SECOND, WATER YEAR OCTOBER 1984 TO SEPTEMBER 1985 DAILY MEAN VALUES

\begin{tabular}{|c|c|c|c|c|c|c|c|c|c|c|c|c|}
\hline DAY & OCT & NOV & DEC & $\mathrm{JAN}$ & FEB & MAR & APR & MAY & JUN & JUL & $\mathrm{AUG}$ & SEP \\
\hline 1 & 0.00 & 0.00 & 0.00 & 0.00 & 00.00 & 00.00 & $\mathrm{e} 0.00$ & 0.00 & 0.00 & 0.00 & 0.00 & 0.00 \\
\hline 2 & $e .01$ & .00 & .00 & .00 & $e .00$ & $e .00$ & 0.00 & .00 & .00 & .00 & .00 & .00 \\
\hline 3 & .00 & .00 & .00 & .00 & e.00 & e.00 & e.00 & .00 & e.00 & .00 & .00 & .00 \\
\hline 4 & .00 & .00 & .00 & .00 & $e .00$ & e.00 & 8.00 & .00 & $e .00$ & .00 & .00 & .00 \\
\hline 5 & .00 & .00 & .00 & .00 & $e .00$ & $e .00$ & $e .00$ & .00 & $e .00$ & .00 & .00 & .00 \\
\hline 6 & .00 & .00 & .00 & .00 & e.00 & $e .00$ & $e .00$ & .00 & 8.00 & .00 & .00 & .00 \\
\hline 7 & .00 & .00 & .00 & .00 & $e .00$ & $e .00$ & 0.00 & .00 & $e .00$ & .00 & .00 & .00 \\
\hline 8 & .00 & .00 & .00 & .00 & e.00 & $e .00$ & $e .00$ & .00 & $e .00$ & .00 & .00 & .00 \\
\hline 9 & .00 & .00 & .00 & 2.00 & e.00 & $e .00$ & $e .00$ & .00 & $\mathrm{e} .00$ & .00 & .00 & .00 \\
\hline 10 & .00 & .00 & .00 & $e .00$ & $e .00$ & e.00 & $e .00$ & .00 & $e .00$ & .00 & .00 & .00 \\
\hline 11 & .00 & .00 & .00 & $e .00$ & $e .00$ & $e .00$ & e.00 & .00 & 8.00 & .00 & .00 & .00 \\
\hline 12 & .00 & .00 & .00 & 8.00 & e.00 & e.00 & $e .00$ & .00 & $e .00$ & .00 & .00 & .00 \\
\hline 13 & .00 & .00 & .00 & e.00 & $e .00$ & $e .00$ & 0.00 & .00 & $e .00$ & .00 & .00 & .00 \\
\hline 14 & .00 & .00 & .00 & $e .00$ & e.01 & $e .00$ & e.00 & .00 & $e .00$ & .00 & .00 & .00 \\
\hline 15 & .00 & .00 & .00 & $e .00$ & e.08 & $e .00$ & $e .00$ & .00 & 0.00 & .00 & .00 & .00 \\
\hline 16 & .00 & .00 & .00 & ๔.01 & e. 10 & e.00 & 0.00 & .00 & $e .00$ & .00 & .00 & .00 \\
\hline 17 & .00 & .00 & .00 & $\mathrm{e.05}$ & e.05 & $e .00$ & $e .00$ & .00 & $e .00$ & .00 & .00 & .00 \\
\hline 18 & .00 & .00 & .00 & e.05 & $e .05$ & e.00 & .00 & .00 & $e .00$ & .00 & .00 & .00 \\
\hline 19 & .00 & .00 & .00 & $e .01$ & e.05 & $e .00$ & .00 & .00 & $e .00$ & .00 & .00 & .00 \\
\hline 20 & .00 & .00 & .00 & $e .06$ & $e .00$ & $e .00$ & .00 & .00 & $e .00$ & .00 & .00 & .00 \\
\hline 21 & .00 & .00 & .00 & $e .20$ & $e .00$ & .00 & .00 & .00 & e.00 & .00 & .00 & .00 \\
\hline 22 & .00 & .00 & .00 & $\mathrm{e.04}$ & $e .00$ & $e .00$ & .00 & .00 & $e .00$ & .00 & .00 & .00 \\
\hline 23 & .00 & .00 & .00 & 0.00 & $e .00$ & $e .00$ & .00 & .00 & $e .00$ & .00 & .00 & .00 \\
\hline 24 & .00 & .00 & .00 & 0.00 & $e .00$ & $e .00$ & .00 & .00 & $e .00$ & .00 & .00 & .00 \\
\hline 25 & .00 & .00 & .00 & $e .00$ & $e .00$ & $e .00$ & .00 & .00 & $e .00$ & .00 & .00 & .00 \\
\hline 26 & .00 & .00 & .00 & $e .00$ & 8.00 & e.00 & .00 & .00 & e.00 & .00 & .00 & .00 \\
\hline 27 & .00 & .00 & .00 & 8.00 & $e .00$ & $e .00$ & .00 & .00 & $e .00$ & .00 & .00 & .00 \\
\hline 28 & .00 & .00 & .00 & $e .00$ & $e .00$ & $e .00$ & .00 & .00 & $e .00$ & .00 & .00 & .00 \\
\hline 29 & .00 & .00 & .00 & 0.00 & - & 0.00 & .00 & .00 & .00 & .00 & .00 & .00 \\
\hline 30 & .00 & .00 & .00 & $e .00$ & -- & 8.00 & .00 & .00 & .00 & .00 & .00 & .00 \\
\hline 31 & .00 & - & .00 & $e .00$ & - & $e .00$ & -- & .00 & - & .00 & .00 & $=$ \\
\hline COTAL & 0.01 & 0.000 & .00 & 0.42 & 0.34 & 0.00 & 0.00 & 0.00 & 0.00 & 0.00 & 0.00 & 0.00 \\
\hline MEAN & .000 & .000 & .000 & .014 & .012 & .000 & .000 & .000 & .000 & .000 & .000 & .000 \\
\hline MAX & .01 & .00 & .00 & .20 & .10 & .00 & .00 & .00 & .00 & .00 & .00 & .00 \\
\hline $\mathrm{MIN}$ & .00 & .00 & .00 & .00 & .00 & .00 & .00 & .00 & .00 & .00 & .00 & .00 \\
\hline AC-FT & .02 & .00 & .00 & .8 & .7 & .00 & .00 & .00 & .00 & .00 & .00 & .00 \\
\hline
\end{tabular}

WTR YR 1985 TOTAL 0.77 MEAN 0.002 MAX 0.20 MIN 0.00 AC-FT 1.5 


\section{AMARGOSA DESERT BASIN}

\section{FORTYMILE WASH AT NARROWS,} NEVADA TEST SITE, NEVADA

LOCATION.--Lat $36^{\circ} 53^{\circ} 13^{\prime \prime}$, long $116^{\circ} 22^{\prime} 50^{\prime \prime}$, Nevada State Coordinates $777933 \mathrm{~N}, 583671 \mathrm{E}$, Nye County, Hydrologic Unit 18090202 , on Nevada Test \$ite, on left bank 5 mi upstream from H Road crossing Fortymile Wash, 19 mi north of intersection between U.S. Route 95 and State Route 373 , and 27 mi northwest of Mercury.

DRAINAGE AREA.--258 $\mathrm{mi}^{2}$.

PERIOD OF RECORD.--September 21, 1983, to current year.

GAGE.--Water-stage recorder. Elevation of gage is $3,680 \mathrm{ft}$ above sea level, from topographic map.

REMARKS.--Records poor. Storage rain gage at station.

EXTREMES FOR PERIOD OF RECORD.--Maximum discharge, $730 \mathrm{ft}^{3} / \mathrm{s}$, July 21,1984 , on basis of slope-area measurement of peak flow, gage height, $5.31 \mathrm{ft}$, from recorded record. Outside high watermark of $6.29 \mathrm{ft}$; no flow most days, most years.

EXTREMES OUTSIDE PERIOD OF RECORD.--Maximum discharge, 1,520 ft/s (estimated), March 3, 1983, from slope-conveyance measurement made May 4, 1983.

EXTREMES FOR CURRENT PERIOD.--September 1983: No flow September 21 to September 30.

DISCHARGE, IN CUBIC FEET PER SECOND, WATER YEAR OCTOBER 1982 TO SEPTEMBER 1983 DAILY MEAN VALUES

\begin{tabular}{|c|c|c|c|c|c|c|c|c|c|c|c|c|}
\hline DAY & OCT & NOV & $\mathrm{DEC}$ & JAN & FEB & MAR & APR & MAY & JUN & JUL & AUG & SEP \\
\hline 1 & $\ldots$ & $\ldots$ & $\ldots$ & $\ldots$ & --. & -.. & $\ldots$ & --- & $\ldots$ & $\cdots$ & -.- & -.. \\
\hline 2 & $\ldots$ & $\ldots$ & $\ldots$ & $\ldots$ & --- & --- & $\ldots$ & --- & $\cdots$ & $\ldots$ & --- & -.. \\
\hline 3 & $\ldots$ & $\cdots$ & -- & - & $\ldots$ & 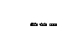 & $\ldots$ & $\cdots$ & --. & $\ldots$ & $\ldots$ & -.. \\
\hline 4 & $\cdots$ & $\cdots$ & $\cdots$ & $\ldots$ & --- & -.. & $\ldots$ & -- & $\ldots$ & $\cdots$ & --. & $\cdots$ \\
\hline 5 & -- & --. & --. & $\ldots$ & $\cdots$ & -.. & $\cdots$ & --- & $\cdots$ & -.. & $\cdots$ & $\cdots$ \\
\hline 6 & $-\ldots$ & --. & $\ldots$ & $\ldots$ & $\ldots$ & ... & $\ldots$ & $\ldots$ & --- & --. & $\cdots$ & -.. \\
\hline 7 & -- & -.- & $\cdots$ & $\ldots$ & --. & $\ldots$ & -.. & $\ldots$ & --. & --. & $\ldots$ & --- \\
\hline 8 & $\ldots$ & --. & -.. & $\ldots$ & --. & $\ldots$ & -.- & -- & $\ldots$ & --. & -.- & $\cdots$ \\
\hline 9 & -- & $\ldots$ & -- & $\ldots$ & -- & --. & $\cdots$ & --- & $\cdots$ & $\cdots$ & $\cdots$ & $\cdots$ \\
\hline 10 & $\cdots$ & -- & -.. & $\cdots$ & -- & $\cdots$ & $\cdots$ & --- & $\cdots$ & $\ldots$ & $\cdots$ & $\cdots$ \\
\hline 11 & -..- & -.- & $\ldots$ & $\ldots$ & $\ldots$ & -..- & .... & $\ldots$ & --- & $\ldots$ & $\ldots$ & $\ldots$ \\
\hline 12 & -.. & -.. & --. & $\ldots$ & $\cdots$ & $\ldots$ & $\ldots$ & -- & -.. & $\ldots$ & $\cdots$ & -.. \\
\hline 13 & $\ldots$ & --. & --. & $\ldots$ & -.- & $\ldots$ & $\ldots$ & $\ldots$ & --- & $\ldots$ & -.- & $\ldots$ \\
\hline 14 & $\ldots$ & $\cdots$ & $\cdots$ & $\ldots$ & --- & $\cdots$ & $\cdots$ & -.. & -- & $\cdots$ & $\cdots$ & $\cdots$ \\
\hline 15 & -.- & $\ldots$ & $\ldots$ & ... & -- & -.. & $\cdots$ & --- & $-\cdots$ & $\cdots$ & --- & -.- \\
\hline 16 & --- & -.- & --. & $\ldots$ & $\ldots$ & -.. & ... & ... & -.. & $\ldots$ & -.. & $\ldots$ \\
\hline 17 & $\ldots$ & $\ldots$ & $\ldots$ & $\ldots$ & $\cdots$ & -- & -.. & --- & $\cdots$ & --. & -.. & -.. \\
\hline 18 & $\cdots$ & $\cdots$ & $\cdots$ & $\ldots$ & -- & -- & --. & $\cdots$ & $\cdots$ & -- & --- & -- \\
\hline 19 & --. & $\ldots$ & -- & $\ldots$ & $\cdots$ & --. & $\ldots$ & -.. & -- & $\ldots$ & -.- & -- \\
\hline 20 & $\cdots$ & $\cdots$ & $\cdots$ & $\cdots$ & $-\cdots$ & $\cdots$ & $\cdots$ & $\cdots$ & --- & --. & $\ldots$ & -- \\
\hline 21 & ... & -- & $\ldots$ & $\ldots$ & $\ldots$ & ... & .-. & ... & $-\cdots$ & ... & ...- & 0.00 \\
\hline 22 & -.. & --. & $\ldots$ & $\ldots$ & -.. & -.. & $\cdots$ & -.. & 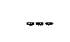 & --. & $\ldots$ & .00 \\
\hline 23 & $\ldots$ & $\ldots$ & $\ldots$ & $\ldots$ &.- & -.. & -- & $\ldots$ & $\ldots$ & $\ldots$ & $\ldots$ & .00 \\
\hline 24 & -.- & --. & -.. & -.. & --. & --. & -.. & ... & $\ldots$ & $\ldots$ & -.. & .00 \\
\hline 25 & $\ldots$ & $\ldots$ & -- & $\ldots$ & $\cdots$ &.-- & $\ldots$ & $\cdots$ & --- & $\ldots$ & $\cdots$ & .00 \\
\hline 26 & $\ldots$ & $\cdots$ & --- & -.- & --- & -.. & -.. & $\ldots$ & $-\ldots$ & --. & $\cdots$ & .00 \\
\hline 27 & -.. & -..- & -.. & -.. & $-\ldots$ & -.- & $\ldots$ & $\ldots$ & --- & --- & -.. & .00 \\
\hline 28 & $\ldots$ & $\ldots$ & $\ldots$ & $\ldots$ & $\ldots$ & --. & $\ldots$ & ... & - & $\ldots$ & $\ldots$ & .00 \\
\hline 29 & -.- & --- & --. & ... & --- & -- & -.. & $\ldots$ & -- & --. & --. & .00 \\
\hline 30 & --- & -- & - & -.- & $\ldots$ & -- & -- & --- & -- & $\cdots$ & - & .00 \\
\hline 31 & $\ldots$ & -- & -.- & $\ldots$ & s.. & -.. & $\cdots$ & $\cdots$ & --- & $\cdots$ & -.. & - \\
\hline TOTAL & $\ldots$ & $\ldots$ & $\ldots$ & $\ldots$ & $\ldots$ & --- & -.. & $\ldots$ & -.- & -.- & -- & $\ldots$ \\
\hline MEAN & $\ldots$ & --- & $\ldots$ & ... & --- & ..- & -- & -.- & -.. & --- & $-\infty$ & -- \\
\hline MAX & $\ldots$ & -.. & -.. & $\ldots$ & -- & --- & $\cdots$ & $\ldots$ & --- & -- & --- & -- \\
\hline MIN & $\ldots$ & $\cdots$ & -.. & $\ldots$ & $\ldots$ & $\cdots$ & $\cdots$ & $\ldots$ & $\cdots$ & $\cdots$ & --- & -- \\
\hline AC-FT & --. & $\ldots$ & -.- & -. & -.- & -.. & $-\cdots$ & -.. & $\ldots$ & -- & $\ldots$ &.-- \\
\hline
\end{tabular}




\begin{tabular}{|c|c|c|c|c|c|c|c|c|c|c|c|c|}
\hline \multicolumn{13}{|c|}{ AMARGOSA DESERT BASIN } \\
\hline \multicolumn{13}{|c|}{$\begin{array}{l}10251250 \text { FORTYMLE WASH AT NARROWS, } \\
\text { NEVADA TEST SITE, NEVADA }\end{array}$} \\
\hline \multicolumn{13}{|c|}{$\begin{array}{l}\text { LOCA1ION --Lat } 36^{\circ} 53^{\prime} 13^{\prime} \text {, long } 116^{\prime} 22^{\prime} 50^{\prime} \text {, Nevada State Coordinates } 777933 N^{\prime}, 583671 \mathrm{E} \text {, Nye County, Hydrologic Unit } \\
18090202 \text {, on Nevada Test Site, on left bank } 5 \mathrm{mi} \text { upstream from H Road crossing Fortymile Wash, } 19 \mathrm{mi} \text { north of intersection } \\
\text { between U S Route } 95 \text { and State Route } 373 \text {, and } 27 \mathrm{~m} \text { northwest of Mercury }\end{array}$} \\
\hline \multicolumn{13}{|c|}{ DRAINAGE AREA $--258 \mathrm{mi}^{2}$} \\
\hline \multicolumn{13}{|c|}{ PERIOD OF RECORD --September 21, 1983, to current year } \\
\hline \multicolumn{13}{|c|}{ GAGE -- Water-stage recorder Elevation of gage is $3,680 \mathrm{ft}$ above sea level, from topographic map } \\
\hline \multicolumn{13}{|c|}{ REMARKS --Records poor Storage rain gage at station } \\
\hline \multicolumn{13}{|c|}{$\begin{array}{l}\text { EXTREMES FOR PERIOD OF RECORD --Maximum discharge, } 730 \mathrm{ft}^{3} / \mathrm{s} \text {, July } 21,1984 \text {, on basis of slope-area measurement } \\
\text { of peak flow, gage height, } 531 \mathrm{ft} \text {, from recorded record Outside high watermark of } 629 \mathrm{ft} \text {, no flow most days, most years }\end{array}$} \\
\hline \multicolumn{13}{|c|}{$\begin{array}{l}\text { EXTREMES OUTSIDE PERIOD OF RECORD --Maximum dischurge, } 1,520 \mathrm{ft}^{3} / \mathrm{s} \text { (estumated), March 3, 1983, from } \\
\text { slope-conveyance measurement made May } 4,1983\end{array}$} \\
\hline \multicolumn{13}{|c|}{$\begin{array}{l}\text { EXTREMES FOR CURRENT PERIOD --Water year } 1984 \text { Maximum discharge, } 730 \mathrm{ft}^{3} / \mathrm{s} \text {, July } 21 \text {, on basis of slope-area } \\
\text { measurement of peak flow, gage height, } 531 \mathrm{ft} \text {, from recorded record Outside high watermark of } 629 \mathrm{ft} \text {, no flow most days }\end{array}$} \\
\hline \multicolumn{13}{|c|}{$\begin{array}{c}\text { DISCHARGE, IN CUBIC FEET PER SECOND, WATER YEAR OCTOBER } 1983 \text { TO SEPTEMBER } 1984 \\
\text { DAILY MEAN VALUES }\end{array}$} \\
\hline DAY & OCT & NOV & DEC & JAN & FEB & MAR & APR & MAY & JUN & JUL & $A \cup G$ & SEP \\
\hline 1 & 000 & 000 & 000 & 000 & 000 & 000 & 000 & 000 & 000 & 000 & 000 & 000 \\
\hline 2 & 00 & 00 & 00 & 00 & 00 & 00 & 00 & 00 & 00 & 00 & 00 & 00 \\
\hline 3 & 00 & 00 & 00 & 00 & 00 & 00 & 00 & 00 & 00 & 00 & 00 & 00 \\
\hline 4 & 00 & 00 & 00 & 00 & 00 & 00 & 00 & 00 & 00 & 00 & 00 & 00 \\
\hline 5 & 00 & 00 & 00 & 00 & 00 & 00 & 00 & 00 & 00 & 00 & 00 & 00 \\
\hline 6 & 00 & 00 & 00 & 00 & 00 & 00 & 00 & 00 & 00 & 00 & 00 & 00 \\
\hline 7 & 00 & 00 & 00 & 00 & 00 & 00 & 00 & 00 & 00 & 00 & 00 & 00 \\
\hline 8 & 00 & 00 & 00 & 00 & 00 & 00 & 00 & 00 & 00 & 00 & 00 & 00 \\
\hline 9 & 00 & 00 & 00 & 00 & 00 & 00 & 00 & 00 & 00 & 00 & 00 & 00 \\
\hline 10 & 00 & 00 & 00 & 00 & 00 & 00 & 00 & 00 & 00 & 00 & 00 & 00 \\
\hline 11 & 00 & 00 & 00 & 00 & 00 & 00 & 00 & 00 & 00 & 00 & 00 & 00 \\
\hline 12 & 00 & 00 & 00 & 00 & 00 & 00 & 00 & 00 & 00 & 00 & 00 & 00 \\
\hline 13 & 00 & 00 & 00 & 00 & 00 & 00 & 00 & 00 & 00 & 00 & 00 & 00 \\
\hline 14 & 00 & 00 & 00 & 00 & 00 & 00 & 00 & 00 & 00 & 00 & 00 & 00 \\
\hline 15 & 00 & 00 & 00 & 00 & 00 & 00 & 00 & 00 & 00 & 00 & e37 & 00 \\
\hline 16 & 00 & 00 & 00 & 00 & 00 & 00 & 00 & 00 & 00 & 00 & e 06 & 00 \\
\hline 17 & 00 & 00 & 00 & 00 & 00 & 00 & 00 & 00 & 00 & 00 & 00 & 00 \\
\hline 18 & 00 & 00 & 00 & 00 & 00 & 00 & 00 & 00 & 00 & 00 & e4 4 & 00 \\
\hline 19 & 00 & 00 & 00 & 00 & 00 & 00 & 00 & 00 & 00 & 00 & es3 & 00 \\
\hline 20 & 00 & 00 & 00 & 00 & 00 & 00 & $\infty$ & 00 & 00 & 00 & 00 & 00 \\
\hline 21 & 00 & 00 & 00 & 00 & 00 & 00 & 00 & 00 & 00 & e56 & 00 & 00 \\
\hline 22 & 00 & 00 & 00 & 00 & 00 & 00 & 00 & 00 & 00 & e10 & 00 & 00 \\
\hline 23 & 00 & 00 & 00 & 00 & 00 & 00 & 00 & 00 & 00 & e 36 & 00 & 00 \\
\hline 24 & 00 & 00 & 00 & 00 & 00 & 00 & 00 & 00 & 00 & 00 & 00 & 00 \\
\hline 25 & 00 & 00 & 00 & 00 & 00 & 00 & 00 & 00 & 00 & 00 & 00 & 00 \\
\hline 26 & 00 & 00 & 00 & 00 & 00 & 00 & 00 & 00 & 00 & 00 & 00 & 00 \\
\hline 27 & 00 & 00 & 00 & 00 & 00 & 00 & 00 & 00 & 00 & 00 & 00 & 00 \\
\hline 28 & $\infty 0$ & 00 & 00 & 00 & 00 & 00 & 00 & 00 & 00 & 00 & 00 & 00 \\
\hline 29 & 00 & 00 & 00 & 00 & 00 & 00 & 00 & 00 & 00 & 00 & 00 & 00 \\
\hline 30 & 00 & 00 & 00 & 00 & -- & 00 & 00 & 00 & 00 & 00 & 00 & 00 \\
\hline 31 & 00 & -- & 00 & 00 & -- & 00 & -- & 00 & - & 00 & 00 & -. \\
\hline TOTAL & 000 & 000 & 000 & 000 & 000 & 000 & 000 & 000 & 000 & 6636 & 6616 & 000 \\
\hline MEAN & 000 & 000 & 000 & 000 & 000 & 000 & 000 & 000 & 000 & 214 & 197 & 000 \\
\hline MAX & 00 & 00 & 00 & 00 & 00 & 00 & 00 & 00 & 00 & 56 & 53 & 00 \\
\hline MIN & 00 & 00 & 00 & 00 & 00 & 00 & 00 & 00 & 00 & 00 & 00 & 00 \\
\hline $\mathrm{AC}-\mathrm{FT}$ & 00 & 00 & 00 & 00 & 00 & 00 & 00 & 00 & 00 & 132 & 121 & 00 \\
\hline
\end{tabular}

WTR YR 1984 TOTAL 12752 MEAN 035 MAX 56 MIN 000 AC-FT 253 


\section{AMARGOSA DESERT BASIN \\ 10251250 FORTYMILE WASH AT NARROWS, NEVADA TEST SITE, NEVADA}

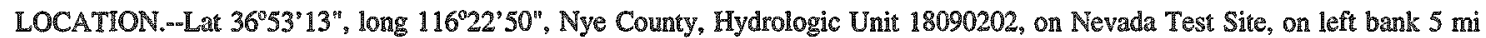
upsiream from $H$ Road crossing Fortymile Wash, 19 mi north of intersection between U.S. Route 95 and State Route 373 , and 27 mi northwest of Mercury.

DRAINAGE AREA.-- $258 \mathrm{mi}^{2}$.

PERIOD OF RECORD.--September 21, 1983, to current year.

GAGE.-Water-stage recorder. Elevation of gage is $3,680 \mathrm{ft}$ above sea level, from topographic map.

REMARKS.--Records poor. Storage rain gage at station.

EXTREMES FOR PERIOD OF RECORD.--Maximum discharge, $730 \mathrm{f}^{3} / \mathrm{s}$, July 21,1984 , on basis of slope-area measurement of peak flow, gage height, $5.31 \mathrm{ft}$, from recorded record. Outside high watermark of $6.29 \mathrm{ft}$; no flow most days, most years.

EXTREMES OUTSIDE PERIOD OF RECORD.--Maximum discharge, 1,520 $\mathrm{ft}^{3} / \mathrm{s}$ (estimated), March 3, 1983, from slopeconveyance measurement made May 4, 1983.

EXTREMES FOR CURRENT PERIOD.--Water year 1985: Maximum discharge, $11.6 \mathrm{ft}^{3} / \mathrm{s}$ (estimated), July 20, gage height, $4.03 \mathrm{ft}$; no flow most days.

DISCHARGE, IN CUBIC FEET PER SECOND, WATER YEAR OCTOBER 1984 TO SEPTEMBER 1985

DAILY MEAN VALUES

\begin{tabular}{|c|c|c|c|c|c|c|c|c|c|c|c|c|}
\hline DAY & OCT & NOV & DEC & JAN & FEB & MAR & APR & MAY & $J U N$ & JUL & $A \cup G$ & SEP \\
\hline 1 & 0.00 & 0.00 & 0.00 & 0.00 & 0.00 & 0.00 & 0.00 & 0.00 & 0.00 & 0.00 & 0.00 & 0.00 \\
\hline 2 & .00 & .00 & .00 & .00 & .00 & .00 & .00 & .00 & .00 & .00 & .00 & .00 \\
\hline 3 & .00 & .00 & .00 & .00 & .00 & .00 & .00 & .00 & .00 & .00 & .00 & .00 \\
\hline 4 & .00 & .00 & .00 & .00 & .00 & .00 & .00 & .00 & .00 & .00 & .00 & .00 \\
\hline 5 & .00 & .00 & .00 & .00 & .00 & .00 & .00 & .00 & .00 & .00 & .00 & .00 \\
\hline 6 & .00 & .00 & .00 & .00 & .00 & .00 & .00 & .00 & .00 & .00 & .00 & .00 \\
\hline 7 & .00 & .00 & .00 & .00 & .00 & .00 & .00 & .00 & .00 & .00 & .00 & .00 \\
\hline 8 & .00 & .00 & .00 & .00 & .00 & .00 & .00 & .00 & .00 & .00 & .00 & .00 \\
\hline 9 & .00 & .00 & .00 & .00 & .00 & .00 & .00 & .00 & .00 & .00 & .00 & .00 \\
\hline 10 & .00 & .00 & .00 & .00 & .00 & .00 & .00 & .00 & .00 & .00 & .00 & .00 \\
\hline 11 & .00 & .00 & .00 & .00 & .00 & .00 & .00 & .00 & .00 & .00 & .00 & .00 \\
\hline 12 & .00 & .00 & .00 & .00 & .00 & .00 & .00 & .00 & .00 & .00 & .00 & .00 \\
\hline 13 & .00 & .00 & .00 & .00 & .00 & .00 & .00 & .00 & .00 & .00 & .00 & .00 \\
\hline 14 & .00 & .00 & .00 & .00 & .00 & .00 & .00 & .00 & .00 & .00 & .00 & .00 \\
\hline 15 & .00 & .00 & .00 & .00 & .00 & .00 & .00 & .00 & .00 & .00 & .00 & .00 \\
\hline 16 & .00 & .00 & .00 & .00 & .00 & .00 & .00 & .00 & .00 & .00 & .00 & .00 \\
\hline 17 & .00 & .00 & .00 & .00 & .00 & .00 & .00 & .00 & .00 & .00 & .00 & .00 \\
\hline 18 & .00 & .00 & .00 & .00 & .00 & .00 & .00 & .00 & .00 & .00 & .00 & .00 \\
\hline 19 & .00 & .00 & .00 & .00 & .00 & .00 & .00 & .00 & .00 & .00 & .00 & .00 \\
\hline 20 & .00 & .00 & .00 & .00 & .00 & .00 & .00 & .00 & .00 & e. 40 & .00 & .00 \\
\hline 21 & .00 & .00 & .00 & .00 & .00 & .00 & .00 & .00 & .00 & .00 & .00 & .00 \\
\hline 22 & .00 & .00 & .00 & .00 & .00 & .00 & .00 & .00 & .00 & .00 & .00 & .00 \\
\hline 23 & .00 & .00 & .00 & .00 & .00 & .00 & .00 & .00 & .00 & .00 & .00 & .00 \\
\hline 24 & .00 & .00 & .00 & .00 & .00 & .00 & .00 & .00 & .00 & .00 & .00 & .00 \\
\hline 25 & .00 & .00 & .00 & .00 & .00 & .00 & .00 & .00 & .00 & .00 & .00 & .00 \\
\hline 26 & .00 & .00 & .00 & .00 & .00 & .00 & .00 & .00 & .00 & .00 & .00 & .00 \\
\hline 27 & .00 & .00 & .00 & .00 & .00 & .00 & .00 & .00 & .00 & .00 & .00 & .00 \\
\hline 28 & .00 & .00 & .00 & .00 & .00 & .00 & .00 & .00 & .00 & .00 & .00 & .00 \\
\hline 29 & .00 & .00 & .00 & .00 & - & .00 & .00 & .00 & .00 & .00 & .00 & .00 \\
\hline 30 & .00 & .00 & .00 & .00 & -- & .00 & .00 & .00 & .00 & .00 & .00 & .00 \\
\hline 31 & .00 & - & .00 & .00 & - & .00 & -- & .00 & -- & .00 & .00 & - \\
\hline TOTAL & 0.00 & 0.00 & 0.00 & 0.00 & 0.00 & 0.00 & 0.00 & 0.00 & 0.00 & 0.40 & 0.00 & 0.00 \\
\hline MEAN & .000 & .000 & .000 & .000 & .000 & .000 & .000 & .000 & .000 & .013 & .000 & .000 \\
\hline MAX & .00 & .00 & .00 & .00 & .00 & .00 & .00 & .00 & .00 & .40 & .00 & .00 \\
\hline MIN & .00 & .00 & .00 & .00 & .00 & .00 & .00 & .00 & .00 & .00 & .00 & .00 \\
\hline$A C-F T$ & .00 & .00 & .00 & .00 & .00 & .00 & .00 & .00 & .00 & .8 & .00 & .00 \\
\hline
\end{tabular}

WTR YR 1985 TOTAL 0.40 MEAN 0.001 MAX 0.40 MIN 0.00 AC-FT 0.8 


\section{AMARGOSA DESERT BASIN \\ 10251255 FORTYMILE WASH NEAR WELL J-13, NEVADA TEST SITE, NEVADA}

LOCATION.-Lat $36^{\circ} 48^{\circ} 27^{\prime \prime}$, long $16^{\circ} 24^{\prime} 01^{\prime \prime}$, Nevada State Coordinates $748995 \mathrm{~N}$, 577984E, Nye County, Hydrologic unit 18090202 , on Nevada Test Site, on right bank $0.85 \mathrm{mi}$ downstream from $\mathrm{H}$ Road crossing Fortymile Wash, 11.5 mi north of intersection between U.S. Route 95 and State Route 373 , and $24 \mathrm{mi}$ west-northwest of Mercury.

DRAINAGE AREA.--304 $\mathrm{mi}^{2}$.

PERIOD OF RECORD.--November 30, 1983, to current year.

GAGE.-Water-stage recorder. Elevation of gage is $3,240 \mathrm{ft}$ above sea level, from topographic map.

REMARKS.--Records poor. Storage rain gage at station.

EXTREMES FOR PERIOD OF RECORD.--Maximum discharge, $1,860 \mathrm{f}^{3} / \mathrm{s}$, July 21 , 1984, on basis of slope-area measurement of peak flow, gage height, $5.82 \mathrm{ft}$, interpolated from high watermark profile. Outside high watermark $6.69 \mathrm{ft}$; no flow most days, most years.

EXTREMES OUTSIDE PERIOD OF RECORD.--Maximum discharge, $570 \mathrm{ft}^{3} / \mathrm{s}$, March 3, 1983, from slope-area measurement made March 9, 1983.

EXTREMES FOR CURRENT PERIOD.--November 1983 to September 1984: Maximum discharge, $1,860 \mathrm{ft}^{3} / \mathrm{s}$, July 21 , on basis of slope-area measurement of peak flow, gage height, $5.82 \mathrm{ft}$, interpolated from high watermark profile. Outside high watermark $6.69 \mathrm{ft}$; no flow most days.

\begin{tabular}{|c|c|c|c|c|c|c|c|c|c|c|c|c|}
\hline \multirow[b]{2}{*}{ DAY } & \multicolumn{12}{|c|}{$\begin{array}{l}\text { DISCHARGE, IN CUBIC FEET PER SECOND, WATER YEAR OCTOBER } 1983 \text { TO SEPTEMBER } 1984 \\
\text { DALY MEAN VALUES }\end{array}$} \\
\hline & OCT & NOV & DEC & $J A N$ & FEB & MAR & $\mathrm{APR}$ & MAY & JUN & JUL & AUG & SEP \\
\hline 1 & $\infty$ & -- & 0.00 & 0.00 & 0.00 & 0.00 & 0.00 & 0.00 & 0.00 & 0.00 & 0.00 & 0.00 \\
\hline 2 & .. & -- & .00 & .00 & .00 & .00 & .00 & .00 & .00 & .00 & .00 & .00 \\
\hline 3 & $\ldots$ & -- & .00 & .00 & .00 & .00 & .00 & .00 & .00 & .00 & .00 & .00 \\
\hline 4 & -. & $-\infty$ & .00 & .00 & .00 & .00 & .00 & .00 & .00 & .00 & .00 & .00 \\
\hline 5 & - & -- & .00 & .00 & .00 & .00 & .00 & .00 & .00 & .00 & .00 & .00 \\
\hline 6 & - & -- & .00 & .00 & .00 & .00 & .00 & .00 & .00 & .00 & .00 & .00 \\
\hline 7 & $\ldots$ & -. & .00 & .00 & .00 & .00 & .00 & .00 & .00 & .00 & .00 & .00 \\
\hline 8 & $\ldots$ & -- & .00 & .00 & .00 & .00 & .00 & .00 & .00 & .00 & .00 & .00 \\
\hline 9 & .. & $=$ & .00 & .00 & .00 & .00 & .00 & .00 & .00 & .00 & .00 & .00 \\
\hline 10 & -- & $\ldots$ & .00 & .00 & .00 & .00 & .00 & .00 & .00 & .00 & .00 & .00 \\
\hline 11 & -- & $\infty$ & .00 & .00 & .00 & .00 & .00 & .00 & .00 & .00 & .00 & .00 \\
\hline 12 & .. & -- & .00 & .00 & .00 & .00 & .00 & .00 & .00 & .00 & .00 & .00 \\
\hline 13 & $\ldots$ & -. & .00 & .00 & .00 & .00 & .00 & .00 & .00 & .00 & .00 & .00 \\
\hline 14 & $\ldots$ & - & .00 & .00 & .00 & .00 & .00 & .00 & .00 & .00 & e.52 & .00 \\
\hline 15 & -. & -- & .00 & .00 & .00 & .00 & .00 & .00 & .00 & .00 & 0.96 & .00 \\
\hline 16 & -. & $\ldots$ & .00 & .00 & .00 & .00 & .00 & .00 & .00 & .00 & .00 & .00 \\
\hline 17 & $\ldots$ & -- & .00 & .00 & .00 & .00 & .00 & .00 & .00 & .00 & .00 & .00 \\
\hline 18 & .. & -- & .00 & .00 & .00 & .00 & .00 & .00 & .00 & .00 & 12 & .00 \\
\hline 19 & -- & - & .00 & .00 & .00 & .00 & .00 & .00 & .00 & .00 & e36 & .00 \\
\hline 20 & -- & -- & .00 & .00 & .00 & .00 & .00 & .00 & .00 & .00 & .00 & .00 \\
\hline 21 & -- & $\ldots$ & .00 & .00 & .00 & .00 & .00 & .00 & .00 & $e 60$ & .00 & .00 \\
\hline 22 & $\ldots$ & $\infty$ & .00 & .00 & .00 & .00 & .00 & .00 & .00 & e20 & .00 & .00 \\
\hline 23 & $\ldots$ & -- & .00 & .00 & .00 & .00 & .00 & .00 & .00 & e.31 & .00 & .00 \\
\hline 24 & $\ldots$ & -. & .00 & .00 & .00 & .00 & .00 & $\begin{array}{l}.00 \\
.00\end{array}$ & .00 & .00 & .00 & .00 \\
\hline 25 & - & -. & .00 & .00 & .00 & .00 & .00 & .00 & .00 & .00 & .00 & .00 \\
\hline 26 & - & $\ldots$ & .00 & .00 & .00 & .00 & .00 & .00 & .00 & .00 & .00 & .00 \\
\hline 27 & $\ldots$ & $\infty$ & .00 & .00 & .00 & .00 & .00 & .00 & .00 & .00 & .00 & .00 \\
\hline 28 & -- & .. & .00 & .00 & .00 & .00 & .00 & .00 & .00 & .00 & .00 & .00 \\
\hline 29 & .. & .. & .00 & 00 & 00 & 00 & 00 & 00 & 00 & .00 & 00 & 00 \\
\hline 30 & 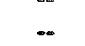 & .00 & .00 & .00 & $=$ & .00 & .00 & .00 & .00 & .00 & .00 & .00 \\
\hline 31 & -. & -- & .00 & .00 & -- & .00 & -. & .00 & - & .00 & .00 & - \\
\hline OTAL & $\infty$ & -. & 0.00 & 0.00 & 0.00 & 0.00 & 0.00 & 0.00 & 0.00 & 80.31 & 49.48 & 0.00 \\
\hline MEAN & -. & -- & .000 & .000 & .000 & .000 & .000 & .000 & .000 & 2.59 & 1.60 & .000 \\
\hline MAX & $\ldots$ & -- & .00 & .00 & .00 & .00 & .00 & .00 & .00 & 60 & 36 & .00 \\
\hline MIN & .- & - & .00 & .00 & .00 & .00 & .00 & .00 & .00 & .00 & .00 & .00 \\
\hline $\mathrm{AC}-\mathrm{FT}$ & - & .. & .00 & .00 & .00 & .00 & .00 & .00 & .00 & $159^{\circ 0}$ & $98^{\circ}$ & .00 \\
\hline
\end{tabular}




\section{AMARGOSA DESERT BASIN \\ 10251255 FORTYMILE WASH NEAR WELL J-13, NEVADA TEST SITE, NEVADA}

LOCATION --Lat $36^{\circ} 48^{\prime} 27$, long $116^{\circ} 24^{\prime} 01$, Nevada State Coordinates 748995N, 577984E, Nye County, Hydrologic unit 18090202, on Nevada Test Site, on right bank $085 \mathrm{mi}$ downstream from H Road crossing Fortymule Wash, $115 \mathrm{mi}$ north of intersection between U S Route 95 and State Route 373, and $24 \mathrm{mi}$ west-northwest of Mercury

DRAINAGE AREA $-304 \mathrm{mi}^{2}$

PERIOD OF RECORD --November 30, 1983, to current year

GAGE --Water-stage recorder Elevation of gage is $3,240 \mathrm{ft}$ above sea level, from topographic map

REMARKS --Records poor Storage rain gage at station

EXTREMES FOR PERIOD OF RECORD --Maximum discharge, 1,860 $\mathrm{ft}^{3} / \mathrm{s}$, July 21,1984 , on basis of slope-area measurement of peak flow, gage height, $582 \mathrm{ft}$, interpolated from high watermark profile Outside high watermark $669 \mathrm{ft}$, no flow most days. most years

EXTREMES OUTSIDE PERIOD OF RECORD --Maximum discharge, $570 \mathrm{ft}^{3} / \mathrm{s}$, March 3, 1983, from slope-area measurement made March 9, 1983

EXTREMES FOR CURRENT PERIOD --Water year 1985 Maximum discharge, $60 \mathrm{ft}^{3} / \mathrm{s}$ (estumated), July 19 , on basis of slopeconveyance measurement of peak flow, gage height, $390 \mathrm{ft}$, no llow most days

DISCHARGE, IN CUBIC FEET PER SECOND, WATER YEAR OCTOBER 1984 TO SEPTEMBER 1985

DAIL Y MEAN VALUES

\begin{tabular}{|c|c|c|c|c|c|c|c|c|c|c|c|c|}
\hline DAY & OCT & NOV & $\mathrm{DEC}$ & JAN & $\mathrm{FEB}$ & MAR & APR & MAY & JUN & JUL & AUG & SEP \\
\hline 1 & 000 & 000 & 000 & 000 & 000 & 000 & 000 & 000 & 000 & 000 & 000 & 000 \\
\hline 2 & 00 & 00 & 00 & 00 & 00 & 00 & 00 & $\infty 0$ & 00 & 00 & 00 & 00 \\
\hline 3 & 00 & 00 & 00 & 00 & 00 & 00 & 00 & 00 & 00 & 00 & 00 & 00 \\
\hline 4 & 00 & 00 & 00 & 00 & 00 & 00 & 00 & 00 & 00 & 00 & 00 & 00 \\
\hline 5 & 00 & 00 & 00 & 00 & 00 & 00 & 00 & $\infty 0$ & 00 & 00 & 00 & 00 \\
\hline 6 & 00 & 00 & 00 & 00 & 00 & 00 & 00 & 00 & 00 & 00 & 00 & 00 \\
\hline 7 & 00 & 00 & 00 & 00 & 00 & 00 & 00 & 00 & 00 & 00 & 00 & 00 \\
\hline 8 & 00 & 00 & 00 & 00 & 00 & 00 & 00 & 00 & 00 & 00 & 00 & 00 \\
\hline 9 & 00 & 00 & 00 & 00 & 00 & 00 & 00 & 00 & 00 & 00 & 00 & 00 \\
\hline 10 & 00 & 00 & 00 & 00 & 00 & 00 & 00 & 00 & 00 & 00 & 00 & 00 \\
\hline 11 & 00 & 00 & 00 & 00 & 00 & 00 & 00 & 00 & 00 & 00 & 00 & 00 \\
\hline 12 & 00 & 00 & 00 & 00 & 00 & 00 & 00 & 00 & 00 & 00 & 00 & 00 \\
\hline 13 & 00 & 00 & 00 & 00 & 00 & 00 & 00 & 00 & 00 & 00 & 00 & 00 \\
\hline 14 & 00 & 00 & 00 & 00 & 00 & 00 & 00 & 00 & 00 & 00 & 00 & 00 \\
\hline 15 & 00 & 00 & 00 & 00 & 00 & $\infty 0$ & 00 & 00 & 00 & 00 & 00 & 00 \\
\hline 16 & 00 & 00 & 00 & 00 & 00 & 00 & 00 & 00 & 00 & 00 & 00 & 00 \\
\hline 17 & 00 & 00 & 00 & 00 & $\infty 0$ & 00 & 00 & 00 & 00 & 00 & 00 & 00 \\
\hline 18 & 00 & 00 & 00 & 00 & 00 & 00 & 00 & 00 & 00 & 00 & 00 & 00 \\
\hline 19 & 00 & 00 & 00 & 00 & 00 & 00 & 00 & 00 & 00 & e 34 & 00 & 00 \\
\hline 20 & 00 & 00 & 00 & 00 & 00 & 00 & 00 & 00 & 00 & 00 & 00 & 00 \\
\hline 21 & 00 & 00 & 00 & 00 & 00 & 00 & 00 & 00 & 00 & 00 & 00 & 00 \\
\hline 22 & 00 & 00 & 00 & 00 & 00 & 00 & 00 & 00 & 00 & 00 & 00 & 00 \\
\hline 23 & 00 & 00 & 00 & 00 & 00 & $\infty$ & 00 & 00 & 00 & 00 & 00 & 00 \\
\hline 24 & 00 & 00 & 00 & 00 & 00 & 00 & 00 & 00 & 00 & 00 & 00 & 00 \\
\hline 25 & 00 & 00 & 00 & 00 & 00 & 00 & 00 & 00 & 00 & 00 & 00 & 00 \\
\hline 26 & 00 & 00 & 00 & 00 & 00 & 00 & 00 & 00 & 00 & 00 & 00 & 00 \\
\hline 27 & 00 & 00 & 00 & 00 & 00 & 00 & 00 & 00 & 00 & 00 & 00 & 00 \\
\hline 28 & 00 & 00 & 00 & 00 & 00 & 00 & 00 & 00 & 00 & 00 & 00 & 00 \\
\hline 29 & 00 & 00 & 00 & 00 & $\ldots$ & 00 & 00 & 00 & 00 & 00 & 00 & 00 \\
\hline 30 & 00 & 00 & 00 & 00 & $\ldots$ & 00 & 00 & 00 & 00 & 00 & 00 & 00 \\
\hline 31 & 00 & $\cdots$ & 00 & 00 & $\cdots$ & 00 & $\cdots$ & 00 & $\cdots$ & 00 & 00 & --- \\
\hline OTAL & 000 & 000 & 000 & 000 & 000 & 000 & 000 & 000 & 000 & 034 & 000 & 000 \\
\hline MEAN & 000 & 000 & 000 & 000 & 000 & 000 & 000 & 000 & 000 & 011 & 000 & 000 \\
\hline MAX & 00 & 00 & 00 & 00 & 00 & 00 & 00 & 00 & 00 & 34 & 00 & 00 \\
\hline MIN & 00 & 00 & 00 & 00 & 00 & 00 & 00 & 00 & 00 & 00 & 00 & 00 \\
\hline$A C-F T$ & 00 & 00 & 00 & 00 & 00 & 00 & 00 & 00 & 00 & 7 & 00 & 00 \\
\hline
\end{tabular}

WTR YR 1985 TOTAL 034 MEAN 0001 MAX 034 MIN 000 AC-FT 07 


\section{AMARGOSA DESERT BASIN \\ 10251258 FORTYMILE WASH NEAR AMARGOSA VALLEY, NEVADA TEST SITE, NEVADA \\ (Formerly published as Fortymile Wash near Lathrop Wells)}

LOCATION.--Lat 36 $40^{\prime} 18^{\prime \prime}$, long 116 26'03", Nevada State Coordinates 699521N, 568183E, Nye County, Hydrologic Unit 18090202, on Nevada Test Site, on left bank 3 mi west-northwest of intersection between U.S. Route 95 and State Route 373 , and 24 mi west of Mercury.

DRAINAGE AREA.--316 mi², revised.

PERIOD OF RECORD..-November 15, 1983, to current year.

REVISED RECORDS.--Squires and Young (1984): Drainage area and name.

GAGE.-Water-stage recorder. Elevation of gage is $2,705 \mathrm{ft}$ above sea level, from topographic map.

REMARKS.--Records poor. Storage rain gage at station.

EXTREMES FOR PERIOD OF RECORD.--Maximum discharge, $1,430 \mathrm{ft}^{3} / \mathrm{s}$, July 22 , 1984, on basis of slope-area measurement of peak flow, gage height, $7.10 \mathrm{ft}$, from high water mark; no flow most days, most years.

EXTREMES OUTSIDE PERIOD OF RECORD.--Maximum discharge, $400 \mathrm{ft}^{3} / \mathrm{s}$ (estimated), March 3, 1983, from slope-conveyance measurement made March 10, 1983.

EXTREMES FOR CURRENT PERIOD.--November 1983 to September 1984: Maximum discharge, 1,430 $\mathrm{ft}^{3} / \mathrm{s}$, July 22 , on basis of slope-area measurement of peak flow, gage height, $7.10 \mathrm{ft}$, from high watermark; no flow most days.

DISCHARGE IN CUBIC FEET PER SECOND, WATER YEAR OCTOBER 1983 TO SEPTEMBER 1984 DAILY MEAN VALUES

\begin{tabular}{|c|c|c|c|c|c|c|c|c|c|c|c|c|}
\hline DAY & $\mathrm{OCT}$ & Nov & $\mathrm{DEC}$ & JAN & FEB & MAR & APR & MAY & JUN & JUL & $\mathrm{AUG}$ & SEP \\
\hline 1 & -. & -- & 0.00 & 0.00 & 0.00 & 0.00 & 0.00 & 0.00 & 0.00 & 0.00 & $e 0.00$ & 0.00 \\
\hline 2 & $-\infty$ & -- & .00 & .00 & .00 & .00 & .00 & .00 & .00 & .00 & e.00 & .00 \\
\hline 3 & - & -- & .00 & .00 & .00 & .00 & .00 & .00 & .00 & .00 & $e .00$ & .00 \\
\hline 4 & -- & -- & .00 & .00 & .00 & .00 & .00 & .00 & .00 & .00 & e.00 & .00 \\
\hline 5 & -- & -- & .00 & .00 & .00 & .00 & .00 & .00 & .00 & .00 & $\mathrm{e} .00$ & .00 \\
\hline 6 & -- & - & .00 & .00 & .00 & .00 & .00 & .00 & .00 & .00 & e.00 & .00 \\
\hline 7 & - & - & .00 & .00 & .00 & .00 & .00 & .00 & .00 & .00 & $\mathrm{e.00}$ & .00 \\
\hline 8 & - & -- & .00 & .00 & .00 & .00 & .00 & .00 & .00 & .00 & $\mathrm{e.00}$ & .00 \\
\hline 9 & - & - & .00 & .00 & .00 & .00 & .00 & .00 & .00 & .00 & 8.00 & .00 \\
\hline 10 & -- & $\ldots$ & .00 & .00 & .00 & .00 & .00 & .00 & .00 & .00 & 8.00 & .00 \\
\hline 11 & - & -- & .00 & .00 & .00 & .00 & .00 & .00 & .00 & .00 & $e .00$ & .00 \\
\hline 12 & $\ldots$ & $\infty$ & .00 & .00 & .00 & .00 & .00 & .00 & .00 & .00 & e.00 & .00 \\
\hline 13 & - & -- & .00 & .00 & .00 & .00 & .00 & .00 & .00 & .00 & $e .00$ & .00 \\
\hline 14 & - & -- & .00 & .00 & .00 & .00 & .00 & .00 & .00 & .00 & $e .00$ & .00 \\
\hline 15 & - & 0.00 & .00 & .00 & .00 & .00 & .00 & .00 & .00 & .00 & e.00 & .00 \\
\hline 16 & - & .00 & .00 & .00 & .00 & .00 & .00 & .00 & .00 & .00 & .50 & .00 \\
\hline 17 & - & .00 & .00 & .00 & .00 & .00 & .00 & .00 & .00 & .00 & e.00 & .00 \\
\hline 18 & $=$ & .00 & .00 & .00 & .00 & .00 & .00 & .00 & .00 & .00 & $\mathrm{e} 3.8$ & .00 \\
\hline 19 & - & .00 & .00 & .00 & .00 & .00 & .00 & .00 & .00 & .00 & e48 & .00 \\
\hline 20 & -- & .00 & .00 & .00 & .00 & .00 & .00 & .00 & .00 & .00 & e. 43 & .00 \\
\hline 21 & - & .00 & .00 & .00 & .00 & .00 & .00 & .00 & .00 & $\mathrm{e} 12$ & e.00 & .00 \\
\hline 22 & - & .00 & .00 & .00 & .00 & .00 & .00 & .00 & .00 & e95 & e. 00 & .00 \\
\hline 23 & - & .00 & .00 & .00 & .00 & .00 & .00 & .00 & .00 & $e 4.5$ & .00 & .00 \\
\hline 24 & -- & .00 & .00 & .00 & .00 & .00 & .00 & .00 & .00 & 0.00 & .00 & .00 \\
\hline 25 & - & .00 & .00 & .00 & .00 & .00 & .00 & .00 & .00 & $e .00$ & .00 & .00 \\
\hline 26 & -- & .00 & .00 & .00 & .00 & .00 & .00 & .00 & .00 & $e .00$ & .00 & .00 \\
\hline 27 & -- & .00 & .00 & .00 & .00 & .00 & .00 & .00 & .00 & $e .00$ & .00 & .00 \\
\hline 28 & -. & .00 & .00 & .00 & .00 & .00 & .00 & .00 & .00 & $e .00$ & .00 & .00 \\
\hline 29 & -.. & .00 & .00 & .00 & .00 & .00 & .00 & .00 & .00 & $e .00$ & .00 & .00 \\
\hline 30 & -. & .00 & .00 & .00 & .- & .00 & .00 & .00 & .00 & $e .00$ & .00 & .00 \\
\hline 31 & - & - & .00 & .00 & -- & .00 & - & .00 & $-\infty$ & $e .00$ & .00 & - \\
\hline TOTAL & - & - & 0.00 & 0.00 & 0.00 & 0.00 & 0.00 & 0.00 & 0.00 & 111.50 & 52.70 & 0.00 \\
\hline MEAN & -. & -- & .000 & .000 & .000 & .000 & .000 & .000 & .000 & 3.60 & 1.70 & .000 \\
\hline MAX & - & - & .00 & .00 & .00 & .00 & .00 & .00 & .00 & 95 & 48.00 & .00 \\
\hline MIN & $-\infty$ & -- & .00 & .00 & .00 & .00 & .00 & .00 & .00 & .00 & .00 & .00 \\
\hline AC-FT & -- & -. & .00 & .00 & .00 & .00 & .00 & .00 & .00 & 221 & 105 & .00 \\
\hline
\end{tabular}


AMARGOSA DESERT BASIN

10251258 FORTYMLLE WASH NEAR AMARGOSA VALLEY, NEVADA TEST SITE, NEVADA

LOCATION.--Lat $36^{\circ} 40^{\prime} 18^{\prime \prime}$, long $16^{\circ} 26^{\prime} 03^{\prime \prime}$, Nevada State Coordinates $699521 \mathrm{~N}$, 568183E, Nye County, Hydrologic Unit 18090202, on Nevada Test Site, on left bank 3 mi west-northwest of intersection between U.S. Route 95 and State Route 373 , and 24 miles west of Mercury.

DRAINAGE AREA.--316 $\mathrm{mi}^{2}$.

PERIOD OF RECORD.--November 15, 1983, to current year. Published as "near Lathrop Wells" by Squires and Young (1984).

REVISED RECORDS.--Squires and Young (1984): Drainage area and name.

GAGE.-Water-stage recorder. Elevation of gage is 2,705 ft above sea level, from topographic map.

REMARKS.--Records poor. Storage rain gage at station.

EXTREMES FOR PERIOD OF RECORD.--Maximum discharge, $1,430 \mathrm{ft}^{3} / \mathrm{s}$, July 22 , 1984, on basis of slope-area measurement of peak flow, gage height, $7.10 \mathrm{ft}$ from high watermark; no flow most days, most years.

EXTREMES OUTSIDE PERIOD OF RECORD.--Maximum discharge, $400 \mathrm{ft} / \mathrm{s}$ (estimated), March 3, 1983, from slope-conveyance measurement made March 10, 1983.

EXTREMES FOR CURRENT PERIOD.--Water year 1985: Maximum discharge, $3.2 \mathrm{ft}^{3} / \mathrm{s}$ (estimated), July 19, on basis of channel characteristics and estimated mean velocity of peak flow, gage height, none; no flow most days.

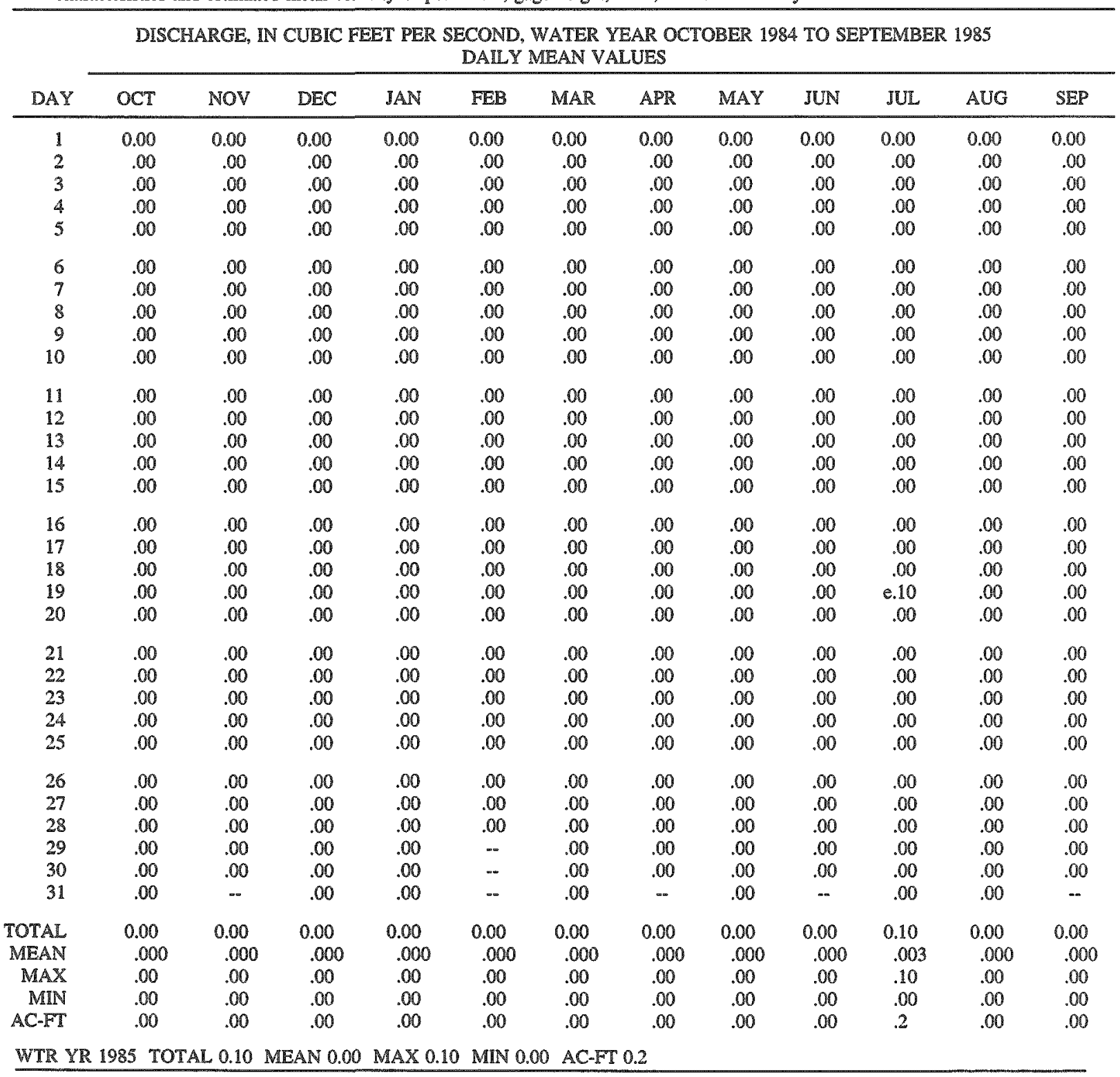


TABLE 3.--Discharge at U.S. Geological Survey crest-stage, partial-record stations

[Abbreviations: Hwy, Highway; lat, latitude; long, longitude; NTS, Nevada Test Site; NV, Nevada]

\begin{tabular}{|c|c|c|c|c|c|c|c|}
\hline \multirow[b]{2}{*}{$\begin{array}{l}\text { Station } \\
\text { number }\end{array}$} & \multirow[b]{2}{*}{ Station name } & \multirow[b]{2}{*}{ Location } & \multirow[b]{2}{*}{$\begin{array}{l}\text { Drainage } \\
\text { area } \\
\text { (square } \\
\text { miles) }\end{array}$} & \multirow[b]{2}{*}{$\begin{array}{l}\text { Period of } \\
\text { record }\end{array}$} & \multicolumn{3}{|c|}{ Measurements } \\
\hline & & & & & Date & $\begin{array}{l}\text { Gage } \\
\text { height } \\
\text { (feet) }\end{array}$ & $\begin{array}{l}\text { Peal } \\
\text { discharge } \\
\text { (cubic fert } \\
\text { per second) }\end{array}$ \\
\hline 10248490 & $\begin{array}{l}\text { Indian Springs Valley } \\
\text { Tributary near Indian } \\
\text { Springs, NV }\end{array}$ & $\begin{array}{l}\text { Lat } 36^{\circ} 34^{\circ} 00^{\prime \prime} \text {, Long } 115^{\circ} 48^{\circ} 40^{\prime \prime} \text {, in } \\
\text { Clark County, at culvert on U.S. } \\
\text { Hwy } 95,8 \text { miles west of Indian } \\
\text { Springs, and } 12 \text { miles east- } \\
\text { southeast of Mercury. }\end{array}$ & $a_{29}$ & $\begin{array}{r}b_{1964-82} \\
1984-85\end{array}$ & $\begin{array}{c}08-15-84 \\
08-19-84 \\
1985\end{array}$ & $\begin{array}{l}2.60 \\
3.97 \\
-\end{array}$ & $\begin{array}{r}67 e \\
c_{130 e} \\
(d)\end{array}$ \\
\hline 10251220 & $\begin{array}{l}\text { Amargosa River near } \\
\text { Beatty, NV }\end{array}$ & $\begin{array}{l}\text { Lat } 36^{\circ} 52^{\prime} 06^{\prime \prime} \text {, long } 116^{\circ} 45^{\prime} 34^{\prime \prime} \text {, in } \\
\text { Nye County, on left bank } 170 \\
\text { feet downstream from Airport } \\
\text { Road, } 2.8 \text { miles south of Beatty } \\
\text { and } 43 \text { miles west-northwest of } \\
\text { Mercury. }\end{array}$ & $a_{470}$ & $\begin{array}{r}b_{1963-68} \\
1969-81 \\
1983-85\end{array}$ & $\begin{array}{c}03-03-83 \\
1984 \\
07-19-85\end{array}$ & $\begin{array}{l}-- \\
--\end{array}$ & $\begin{array}{c}c_{200} \\
(d) \\
5.13\end{array}$ \\
\hline 10251252 & $\begin{array}{l}\text { Yucca Wash near mouth, } \\
\text { NTS, NV (formerly } \\
\text { published as Yucca } \\
\text { Wash) }\end{array}$ & $\begin{array}{l}\text { (Revised) } \\
\text { Lat } 36^{\circ} 51^{\prime} 58^{\prime \prime} \text {, long } 116^{\circ} 23^{\prime} 38^{\prime \prime} \text {, } \\
\text { Nye County, on left bank } 0.35 \\
\text { mile above confluence with } \\
\text { Fortymile Wash, } 26 \text { miles } \\
\text { northwest of Mercury. }\end{array}$ & ${ }^{c} 17.0$ & $1982-85$ & $\begin{array}{l}03-03-83 \\
07-21-84 \\
08-19-84 \\
07-19-85\end{array}$ & $\begin{array}{l}2.72 \\
-- \\
--\end{array}$ & $\begin{array}{c}c_{100 c} \\
c_{940 e} \\
31 e \\
<01\end{array}$ \\
\hline 10251254 & $\begin{array}{l}\text { Drillhole Wash at mouth, } \\
\text { NTS, NV (formerly } \\
\text { published as Drill Hole } \\
\text { Wash) }\end{array}$ & $\begin{array}{l}\text { (Revised) } \\
\text { Lat } 36^{\circ} 49^{\prime} 13^{\prime \prime} \text { long } 116^{\circ} 23^{\prime} 52^{\prime \prime} \text {. } \\
\text { Nye County, on left bank } 0.15 \\
\text { mile above confluence with } \\
\text { Fortymile Wash, } 25 \text { miles west- } \\
\text { northwest of Mercury. }\end{array}$ & $c_{16.3}$ & $1983-85$ & $\begin{array}{c}1983 \\
07-23-84 \\
08-19-84 \\
07-19-85\end{array}$ & $\begin{array}{l}- \\
3.38 \\
- \\
-\end{array}$ & $\begin{array}{l}(d) \\
790 e \\
43 e \\
617 c\end{array}$ \\
\hline 10251256 & $\begin{array}{l}\text { Dune Wash near Busted } \\
\text { Butte, NTS, NV (formerly } \\
\text { published as Busted Butte } \\
\text { Wash) }\end{array}$ & $\begin{array}{l}\text { (Revised) } \\
\text { Lat } 36^{\circ} 47^{\prime} 35^{\prime \prime} \text {, long } 116^{\circ} 24^{\prime} 29^{\prime \prime} \text {, } \\
\text { Nye County, on left bank } 0.8 \\
\text { mile above confluence with } \\
\text { Fortymile Wash, } 24.5 \text { miles } \\
\text { west-northwest of Mercury }\end{array}$ & ${ }^{c} 6.77$ & $1982-85$ & $\begin{array}{c}1983 \\
08-19-84 \\
07-19-85\end{array}$ & $\begin{array}{l}- \\
2.05 \\
--\end{array}$ & $\begin{array}{l}(d) \\
c_{14 e} \\
c_{9}+e^{2}\end{array}$ \\
\hline 10251260 & $\begin{array}{l}\text { Topopah Wash at Little } \\
\text { Skull Mountain, NTS, } \\
\text { NV (formerly published } \\
\text { as Topopah Wash near } \\
\text { Lathrop Wells) }\end{array}$ & $\begin{array}{l}\text { (Revised) } \\
\text { Lat } 36^{\circ} 46^{\circ} 06^{\prime \prime} \text {, long } 116^{\circ} 19^{\prime} 23^{\prime \prime} \text {, } \\
\text { Nye County, on right bank, at } \\
\text { base of Little Skull Mountain, } \\
0.23 \text { mile south of Lathrop Wells } \\
\text { road, } 19 \text { miles west-northwest of } \\
\text { Mercury. }\end{array}$ & $c_{104}$ & $1984-85$ & $\begin{array}{l}07-21-84 \\
07-31-84 \\
08-15-84 \\
08-19-84 \\
11-22-84 \\
12-19-84\end{array}$ & $\begin{array}{l}3.60 \\
3.70 \\
- \\
- \\
- \\
--\end{array}$ & $\begin{array}{r}1316 \\
120 \\
83 \\
531 \\
53 \\
1.06 \\
.106\end{array}$ \\
\hline 10251265 & $\begin{array}{l}\text { Cane Spring Wash Tributary } \\
\text { near Cane Spring, NTS, } \\
\text { NV (formerly published } \\
\text { as Cane Spring Wash } \\
\text { Tributary near Mercury) }\end{array}$ & $\begin{array}{l}\text { (Revised) } \\
\text { Lat } 36^{\circ} 48^{\circ} 27^{\prime \prime}, \text { long } 116^{\circ} 05^{\circ} 41^{\prime \prime} \text {, } \\
\text { Nye County, on left bank } 20 \text { feet } \\
\text { upstream of Cane Spring Road } \\
\text { Crossing, } 0.55 \text { mile north of } \\
\text { Cane Spring and } 11.5 \text { miles } \\
\text { northwest of Mercury. }\end{array}$ & $c_{8.36}$ & $1984-85$ & $\begin{array}{c}07-24-84 \\
1985\end{array}$ & - & $\begin{array}{l}60.54 \mathrm{H}^{\circ} \\
\text { (d) }\end{array}$ \\
\hline 10251270 & $\begin{array}{l}\text { Amargosa River Tributary } \\
\text { near Mercury, NV }\end{array}$ & $\begin{array}{l}\text { Lat } 36^{\circ} 33^{\prime} 40^{\prime \prime} \text {. long } 116^{\circ} 06^{\prime} 00^{\prime \prime} \text {, } \\
\text { Nye County, on left bank at } \\
\text { upstream side of highway culvert } \\
\text { at U.S. Highway } 95,9 \text { miles } \\
\text { southwest of Mercury. }\end{array}$ & 110 & $\begin{array}{r}b_{1968-81} \\
1984.85\end{array}$ & $\begin{array}{l}07-15-84 \\
08-15-84 \\
08-19-84 \\
1985\end{array}$ & $\begin{array}{l}3.03 \\
- \\
\bar{c}_{5.48} \\
\ldots\end{array}$ & $\begin{array}{r}196 \\
26 \varepsilon \\
c_{1,150} \\
(6)\end{array}$ \\
\hline
\end{tabular}


TABLE 3.--Discharge at U.S. Geological Survey crest-stage, partial-record stations--Continued.

\begin{tabular}{|c|c|c|c|c|c|c|c|}
\hline \multirow[b]{2}{*}{$\begin{array}{l}\text { Station } \\
\text { number }\end{array}$} & \multirow[b]{2}{*}{ Station mame } & \multirow[b]{2}{*}{ Location } & \multirow[b]{2}{*}{$\begin{array}{c}\text { Drainage } \\
\text { area } \\
\text { (square } \\
\text { miles) }\end{array}$} & \multirow[b]{2}{*}{$\begin{array}{l}\text { Period of } \\
\text { record }\end{array}$} & \multicolumn{3}{|c|}{ Measurements } \\
\hline & & & & & Date & $\begin{array}{c}\text { Gage } \\
\text { height } \\
\text { (feet) }\end{array}$ & $\begin{array}{c}\text { Peak } \\
\text { discharge } \\
\text { (cubic feet } \\
\text { per second) }\end{array}$ \\
\hline 10251271 & $\begin{array}{l}\text { Amargosa River Tributary } \\
\text { No. I near Johnuie, NV }\end{array}$ & $\begin{array}{l}\text { Lat } 36^{\circ} 27^{\prime} 36^{\prime \prime} \text {, long } 116^{\circ} 06^{\prime} 28^{\prime \prime} \text {, in } \\
\text { Nye County, at culvert on State } \\
\text { Route } 160,3.5 \text { miles northwest } \\
\text { of Johnnie and } 15 \text { miles } \\
\text { southwest of Mercury. }\end{array}$ & 2.21 & $\begin{array}{r}b_{1967-81} \\
1984-85\end{array}$ & $\begin{array}{l}08-84 \\
1985\end{array}$ & $\begin{array}{l}7.23 \\
--\end{array}$ & $\begin{array}{l}c_{95 e} \\
(d)\end{array}$ \\
\hline 10251272 & $\begin{array}{l}\text { Amargosa River Tributary } \\
\text { No. } 2 \text { near Johnnie, NV }\end{array}$ & $\begin{array}{l}\text { Lat } 36^{\circ} 26^{\circ} 09^{\prime \prime}, \text { long } 116^{\circ} 04^{\prime} 28^{\prime \prime} \text {, in } \\
\text { Nye County, at culvert on State } \\
\text { Route } 160,1.2 \text { miles north of } \\
\text { Johnnie and } 16 \text { miles south- } \\
\text { southwest of Mercury. }\end{array}$ & $c_{2.34}$ & $\begin{array}{r}b_{1968-81} \\
1984-85\end{array}$ & $\begin{array}{c}08-84 \\
1985\end{array}$ & $\begin{array}{l}3.45 \\
.-\end{array}$ & $\begin{array}{l}c_{2.0 e} \\
\text { (d) }\end{array}$ \\
\hline
\end{tabular}

Approximate.

b Records prior to reporting period published by U.S. Geological Survey (1964-85) for appropriate water years, and Mloosbumer (1978).

Revised. Revises Squires and Young (1984) and U.S. Geological Survey for water years 1983, 1984, and 1985.

"No evidence of llow during water year.

Estimated. 
TABLE 4.--Discharge at U.S. Geological Survey miscellaneous sites

[Abbreviations: NTS, Nevada Test Site; NV, Nevada]

\begin{tabular}{|c|c|c|c|c|c|c|}
\hline \multirow[b]{2}{*}{$\begin{array}{l}\text { Station } \\
\text { number }\end{array}$} & \multirow[b]{2}{*}{ Station name } & \multirow[b]{2}{*}{$\begin{array}{l}\text { Latitude } \\
\text { (degrees, } \\
\text { minutes, } \\
\text { seconds) }\end{array}$} & \multirow[b]{2}{*}{$\begin{array}{l}\text { Longitude } \\
\text { (degrees, } \\
\text { minutes, } \\
\text { seconds) }\end{array}$} & \multirow[b]{2}{*}{$\begin{array}{l}\text { Drainage } \\
\text { area } \\
\text { (square } \\
\text { miles) }\end{array}$} & \multicolumn{2}{|c|}{ Measurements } \\
\hline & & & & & Date & $\begin{array}{c}\text { Peak } \\
\text { discharge } \\
\text { (cubic feet } \\
\text { per second) }\end{array}$ \\
\hline 10247890 & $\begin{array}{l}\text { KP's Wash at Tippipah } \\
\text { Highway, NTS, NV }\end{array}$ & $37^{\circ} 10^{\prime} 07^{\prime \prime}$ & $116^{\circ} 08^{\circ} 27^{\prime \prime}$ & 1.08 & $\begin{array}{l}07-19-84 \\
08-19-84\end{array}$ & $\begin{array}{l}300 e \\
5.0 e\end{array}$ \\
\hline 10251244 & $\begin{array}{l}\text { Stockade Wash below Stockade } \\
\text { Road, NTS, NV }\end{array}$ & $37^{\circ} 08^{\prime} 59^{\prime \prime}$ & $116^{\circ} 14^{\prime} 45^{\prime \prime}$ & 3.86 & $07-19-84$ & $20 e$ \\
\hline$a_{10251250}$ & $\begin{array}{l}\text { Fortymile Wash at Narrows, } \\
\text { NTS, NV (formerly published } \\
\text { as Fortymile Wash cross- } \\
\text { section } 7 \text { ) }\end{array}$ & $b_{36}^{\circ} 53^{\prime} 13^{\prime \prime}$ & $116^{\circ} 22^{\prime} 50^{\prime \prime}$ & $b_{258}$ & 03-03-83 & $1,520 e$ \\
\hline 102512532 & $\begin{array}{l}\text { Pagany Wash } 2 \text {, (Tributary to } \\
\text { Drill Hole Wash), NTS, NV }\end{array}$ & $36^{\circ} 51^{\prime} 42^{\prime \prime}$ & $116^{\circ} 26^{\prime} 24^{\prime \prime}$ & .73 & $08-19-84$ & $150 e$ \\
\hline 102512533 & $\begin{array}{l}\text { Pagany Wash } 1 \text {, (Tributary to } \\
\text { Drill Hole Wash), NTS, NV }\end{array}$ & $36^{\circ} 51^{\prime} 39^{\prime \prime}$ & $116^{\circ} 26^{\circ} 08^{\prime \prime}$ & .82 & $08-19-84$ & $100 e$ \\
\hline 102512535 & $\begin{array}{l}\text { Drill Hole Wash above Well } \\
\text { UZ-1, NTS, NV }\end{array}$ & $36^{\circ} 52^{\prime} 16^{\prime \prime}$ & $116^{\circ} 27^{\prime} 46^{\prime \prime}$ & .53 & $08-19-84$ & $42 e$ \\
\hline 102512536 & $\begin{array}{l}\text { Drill Hole Wash above Well } \\
\text { UE-25, NTS, NV }\end{array}$ & $36^{\circ} 51^{\circ} 08^{\prime \prime}$ & $116^{\circ} 26^{\circ} 25^{\prime \prime}$ & 2.78 & $08-19-84$ & $17 e$ \\
\hline 102512537 & $\begin{array}{l}\text { Split Wash below Quac Canyon, } \\
\text { NTS, NV }\end{array}$ & $36^{\circ} 50^{\prime} 57^{\prime \prime}$ & $116^{\circ} 26^{\prime} 54^{\prime \prime}$ & .33 & $07-21-84$ & $290 e$ \\
\hline 10251255 & $\begin{array}{l}\text { Fortymile Wash near Well J-13, } \\
\text { NTS, NV }\end{array}$ & $36^{\circ} 48^{\prime \prime} 27^{\prime \prime}$ & $116^{\circ} 24^{\circ} 01 "$ & 304 & $03-03-83$ & $570 e$ \\
\hline${ }^{a} 10251258$ & $\begin{array}{l}\text { Fortymile Wash near Amargosa } \\
\text { Valley, NV (formerly } \\
\text { published as Fortymile Wash } \\
\text { cross-section 1) }\end{array}$ & $b_{36} 40^{\prime} 18^{\prime \prime}$ & $116^{\circ} 26^{\circ} 03^{\prime \prime}$ & $b_{304}$ & $03-03-83$ & $400 e$ \\
\hline 10251266 & $\begin{array}{c}\text { Frenchman Lake Tributary at old } \\
\text { Mercury Highway, NTS, NV }\end{array}$ & $36^{\circ} 43^{\prime} 48^{\prime \prime}$ & $115^{\circ} 59^{\prime} 59^{\prime \prime}$ & 6.34 & $08-15-84$ & $1,100 e$ \\
\hline 10251269 & $\begin{array}{l}\text { Gas Station Wash at Mercury, } \\
\text { NTS, NV }\end{array}$ & $36^{\circ} 39^{\circ} 36^{\prime \prime}$ & $116^{\circ} 00^{\circ} 01^{\prime \prime}$ & .52 & $07-15-84$ & $100 e$ \\
\hline
\end{tabular}

a Published as crest-stage gage in U.S. Geological Survey $(1984$, p. 273 ; water year 1983).

$b$ Revised. Revises U.S. Geological Survey (1984; water year 1983).

e Estimated. 
TABLE 5.--Precipitation data from U.S. Geological Survey network stations

[Abbreviations: NTS, Nevada Test Site; NV, Nevada; RGD, rain gage destroyed; T, trace amount]

\begin{tabular}{|c|c|c|c|c|c|c|}
\hline Stution manus: & $\begin{array}{l}\text { Station } \\
\text { number }\end{array}$ & $\begin{array}{l}\text { Latitude } \\
\text { (degrees, } \\
\text { minutes, } \\
\text { seconds) }\end{array}$ & $\begin{array}{l}\text { Longitude } \\
\text { (degrees, } \\
\text { minutes, } \\
\text { seconds) }\end{array}$ & $\begin{array}{l}\text { Elevation } \\
\text { (feet above } \\
\text { sea level) }\end{array}$ & Period & $\begin{array}{l}\text { Precipifation } \\
\text { (inches) }\end{array}$ \\
\hline 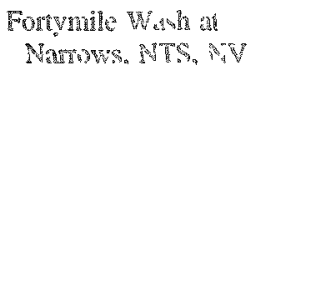 & 10251250 & $36^{\circ} 53^{\prime} 13^{\prime \prime}$ & $116^{\circ} 22^{\prime} 50^{\prime \prime}$ & 3,680 & $\begin{array}{lll}02 / 08 / 84 & \text { TO } 03 / 28 / 84 \\
03 / 28 / 84 & \text { TO } 04 / 05 / 84 \\
04 / 05 / 84 & \text { TO } 07 / 12 / 84 \\
07 / 12 / 84 & \text { TO } 01 / 09 / 85 \\
01 / 09 / 85 & \text { TO } 04 / 30 / 85 \\
04 / 30 / 85 & \text { TO } 06 / 28 / 85 \\
06 / 28 / 85 & \text { TO } 07 / 23 / 85 \\
07 / 23 / 85 & \text { TO } 08 / 22 / 85 \\
08 / 22 / 85 & \text { TO } 09 / 24 / 85\end{array}$ & $\begin{array}{r}0.0 \\
.0 \\
.1 \\
1.7 \\
.2 \\
.1 \\
1.8 \\
.0 \\
.6\end{array}$ \\
\hline 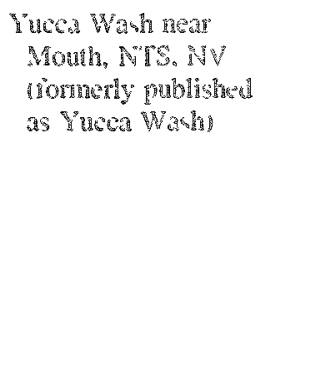 & 10251252 & $r_{36} 51^{\circ} 58^{\circ}$ & $116^{\circ} 23^{\circ} 38^{\prime \prime}$ & 3.590 & $\begin{array}{l}\text { 02/08/84 TO } 03 / 28 / 84 \\
03 / 28 / 84 \text { TO } 05 / 24 / 84 \\
05 / 24 / 84 \text { TO } 07 / 12 / 84 \\
07 / 12 / 84 \text { TO } 08 / 21 / 84 \\
08 / 21 / 84 \text { TO } 10 / 09 / 84 \\
\text { 10/09/84 TO } 11 / 27 / 84 \\
11 / 27 / 84 \text { TO } 01 / 09 / 85 \\
011 / 09 / 85 \text { TO } 04 / 30 / 85 \\
04 / 30 / 85 \text { TO } 07 / 23 / 85 \\
07 / 23 / 85 \text { TO } 08 / 22 / 85 \\
08 / 22 / 85 \text { TO } 09 / 24 / 85\end{array}$ & $\begin{array}{c}0.0 \\
.0 \\
.1 \\
2.4 \\
.0 \\
1.2 \\
.4 \\
.16 \\
2.5 \\
.0 \\
\text { RGD }\end{array}$ \\
\hline 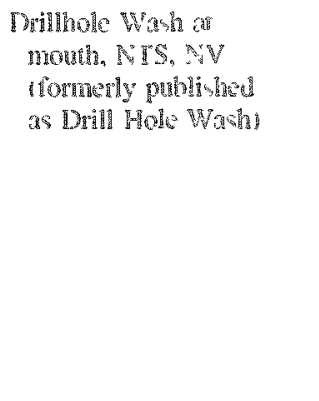 & 10251254 & $r_{36^{\circ} 49^{\prime} 13^{\prime \prime}}$ & $116^{\circ} 23^{\prime} 52^{\prime \prime}$ & 3,320 & $\begin{array}{l}02 / 09 / 84 \text { TO } 05 / 24 / 84 \\
05 / 24 / 84 \text { TO } 07 / 12 / 84 \\
07 / 12 / 84 \text { TO } 07 / 30 / 84 \\
07 / 30 / 84 \text { TO } 07 / 31 / 84 \\
07 / 31 / 84 \text { TO } 08 / 21 / 84 \\
08 / 21 / 84 \text { TO } 10 / 09 / 84 \\
10 / 09 / 84 \text { TO } 11 / 27 / 84 \\
11 / 27 / 84 \text { TO } 01 / 09 / 85 \\
01 / 09 / 85 \text { TO } 04 / 30 / 85 \\
04 / 30 / 85 \text { TO } 07 / 23 / 85 \\
07 / 23 / 85 \text { TO } 08 / 22 / 85 \\
08 / 22 / 85 \text { TO } 09 / 24 / 85\end{array}$ & $\begin{array}{l}0.0 \\
\mathrm{~T} \\
.3 \\
\mathrm{~T} \\
.8 \\
.0 \\
1.2 \\
1.5 \\
.2 \\
.3 \\
.0 \\
.4\end{array}$ \\
\hline 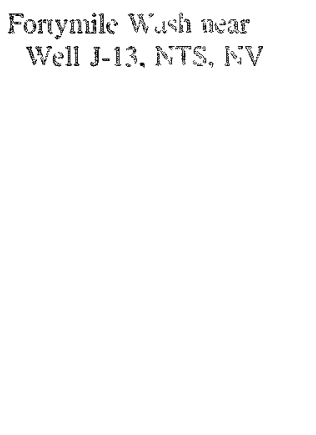 & 10251255 & $36^{\circ} 48^{\prime} 27^{\prime \prime}$ & $116^{\circ} 24^{\prime} 01^{\prime \prime}$ & 3,240 & $\begin{array}{l}06 / 06 / 84 \text { TO } 06 / 15 / 84 \\
06 / 15 / 84 \text { TO } 07 / 23 / 84 \\
07 / 23 / 84 \text { TO } 07 / 31 / 84 \\
07 / 31 / 84 \text { TO } 08 / 15 / 84 \\
08 / 15 / 84 \text { TO } 08 / 22 / 84 \\
08 / 22 / 84 \text { TO } 10 / 09 / 84 \\
10 / 09 / 84 \text { TO } 11 / 27 / 84 \\
11 / 27 / 84 \text { TO } 01 / 09 / 85 \\
01 / 09 / 85 \text { TO } 04 / 30 / 85 \\
04 / 30 / 85 \text { TO } 06 / 28 / 85 \\
06 / 28 / 85 \text { TO } 07 / 23 / 85 \\
07 / 23 / 85 \text { TO } 08 / 22 / 85 \\
08 / 22 / 85 \text { To } 09 / 24 / 85\end{array}$ & $\begin{array}{r}0.0 \\
3.1 \\
.2 \\
1.4 \\
.8 \\
.0 \\
1.3 \\
1.7 \\
.2 \\
.2 \\
.6 \\
.0 \\
.4\end{array}$ \\
\hline 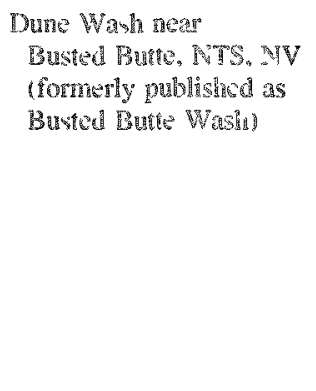 & 10251256 & $r_{36^{\circ}} 47^{\prime} 35^{\prime \prime}$ & $116^{\circ} 24^{\prime} 29^{\prime \prime}$ & 3,270 & $\begin{array}{l}\text { 03/28/84 TO 05/24/84 } \\
05 / 24 / 84 \text { TO } 07 / 13 / 84 \\
07 / 13 / 84 \text { TO } 07 / 30 / 84 \\
07 / 30 / 84 \text { TO } 08 / 21 / 84 \\
08 / 21 / 84 \text { TO } 10 / 09 / 84 \\
10 / 09 / 84 \text { TO } 11 / 27 / 84 \\
11 / 27 / 84 \text { TO } 01 / 09 / 85 \\
01 / 09 / 85 \text { TO } 04 / 30 / 85 \\
04 / 30 / 85 \text { TO } 06 / 28 / 85 \\
06 / 28 / 85 \text { TO } 07 / 23 / 85 \\
07 / 23 / 85 \text { TO } 08 / 22 / 85 \\
08 / 22 / 85 \text { TO } 09 / 24 / 85\end{array}$ & $\begin{array}{r}0.0 \\
.0 \\
.2 \\
.7 \\
.0 \\
1.2 \\
1.9 \\
.2 \\
.1 \\
.4 \\
.0 \\
\text { RGD }\end{array}$ \\
\hline
\end{tabular}


TABLE 5.-Precipitation data from U.S. Geological Survey network stations-continued.

\begin{tabular}{|c|c|c|c|c|c|c|}
\hline Station name & $\begin{array}{c}\text { Station } \\
\text { number }\end{array}$ & $\begin{array}{l}\text { Latinude } \\
\text { (degrees. } \\
\text { minutes, } \\
\text { seconds) }\end{array}$ & $\begin{array}{l}\text { Longitude } \\
\text { (degrees, } \\
\text { minutes, } \\
\text { seconds) }\end{array}$ & $\begin{array}{c}\text { Elevation } \\
\text { (feet above } \\
\text { sea level) }\end{array}$ & Period & 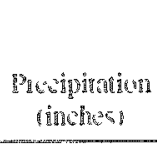 \\
\hline $\begin{array}{l}\text { Fortymile Wash near } \\
\text { Amargosa Valley, NV } \\
\text { (formerly published as } \\
\text { Fortymile Wash near } \\
\text { Lathrop Wells, NV) }\end{array}$ & 10251258 & $36^{\circ} 40^{\prime} 18^{\prime \prime}$ & $116^{\circ} 26^{\circ} 03^{\prime \prime}$ & 2,705 & $\begin{array}{l}\text { 11/27/84 TO 01/10/3: } \\
01 / 10 / 85 \text { TO } 04 / 30 \\
04 / 30 / 85 \text { TO } 06 / 28 / \\
06 / 28 / 85 \text { TO } 07 / 23 / 4 \\
07 / 23 / 85 \text { TO } 09 / 24 / 85\end{array}$ & $\begin{array}{l}1.3 \\
1 \\
0.1 \\
0 \\
3 \\
3\end{array}$ \\
\hline $\begin{array}{l}\text { Topapah Wash at Little } \\
\text { Skull Mountain, NTS, } \\
\text { NV (formerly published } \\
\text { as Topopah Wash near } \\
\text { Lathrop Wells) }\end{array}$ & 10251260 & ${ }^{r} 36^{\circ} 46^{\prime} 06^{\prime \prime}$ & $116^{\circ} 19^{\prime} 23^{\prime \prime}$ & 3.220 & 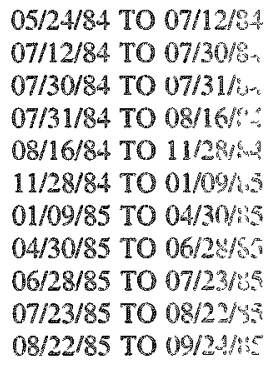 & 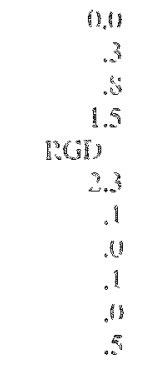 \\
\hline $\begin{array}{l}\text { Rock Valley at U.S. } \\
\text { Highway } 95 \text { near } \\
\text { Amargosa Valley. NV }\end{array}$ & 10251262 & $36^{\circ} 37^{\prime} 35^{\prime \prime}$ & $116^{\circ} 08^{\circ} 31^{\prime \prime}$ & 2,840 & 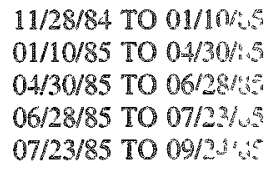 & $\begin{array}{l}1.6 \\
\mathrm{~T} \\
.3 \\
.3 \\
.9\end{array}$ \\
\hline $\begin{array}{l}\text { Cane Spring Wash } \\
\text { Tributary near Cane } \\
\text { Spring, NTS, NV } \\
\text { (formerly published as } \\
\text { Cane Spring Tributary } \\
\text { near Mercury) }\end{array}$ & 10251265 & $r^{3} 6^{\circ} 48^{\prime} 27^{\prime \prime}$ & $116^{\circ} 05^{\circ} 41^{\circ}$ & 3,890 & 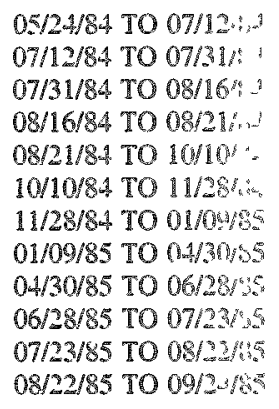 & 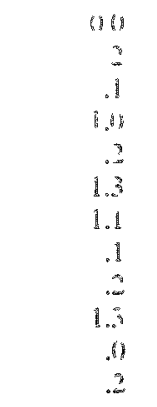 \\
\hline $\begin{array}{l}\text { Amargosa River Tributary } \\
\text { near Mercury, NV }\end{array}$ & 10251270 & $36^{\circ} 33^{\circ} 40^{\prime \prime}$ & $116^{\circ} 06^{\circ} 00^{\prime \prime}$ & 3,425 & $\begin{array}{l}\text { 11/27/84 TO 01/11\%65 } \\
01 / 10 / 85 \text { TO 07/23/45 } \\
07 / 23 / 85 \text { TO 08/22/65 } \\
08 / 22 / 85 \text { TO 09/93/65 }\end{array}$ & $\begin{array}{c}1.0 \\
1.1 \\
.1 \\
1.1\end{array}$ \\
\hline $\begin{array}{l}\text { North Fork Coyote Wash, } \\
\text { NTS, NV }\end{array}$ & NFCW & $36^{\circ} 51^{\prime} 16^{\prime \prime}$ & $116^{\circ} 27^{\circ} 01^{\prime \prime}$ & 4.110 & 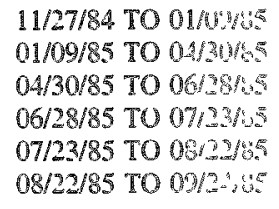 & $\begin{array}{r}1.0 \\
.1 \\
.3 \\
.8 \\
.0 \\
.6 \\
.6\end{array}$ \\
\hline $\begin{array}{l}\text { Stockade Pass, above } \\
\text { Area 12, NTS, NV }\end{array}$ & $\mathbb{S P}$ & $37^{\circ} 09^{\circ} 47^{\prime \prime}$ & $116^{\circ 1} 12^{\circ}\left(3^{\prime \prime}\right.$ & 6,735 & 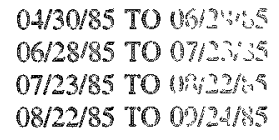 & $\begin{array}{l}6.3 \\
0.3 \\
1.0 \\
10 \\
.03 \\
.3\end{array}$ \\
\hline
\end{tabular}

${ }^{r}$ Revised. 
TABLE 6.--Weather Senice Nuclear Support Office precipitation gaging stations

Station.--MER. Mercury: RV, Rock Valley; 4JA, Jaclass Flats; DRA Desert Rock; CS, Cane Spring; W5B, Well 5B: MV. Mid Valley; UCC. Yucca Lake: 40MN, Fortymile North; TS2, Tippipah Springs: BJY, Buster Jangle Y; A12, Area 12 Mesa; PHS, Public Health Service Farm; PM1, Pahute Mesa \#1;

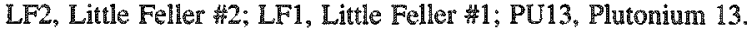

Type of gage: S, storage rain gage; TB, tipping-bucket rain gage; $W$, weighing rain gage.

\begin{tabular}{|c|c|c|c|c|c|c|}
\hline Station & Station name & $\begin{array}{l}\text { Type of } \\
\text { gage }\end{array}$ & $\begin{array}{l}\text { Latitude } \\
\text { (degrees, } \\
\text { minutes, } \\
\text { seconds) }\end{array}$ & $\begin{array}{l}\text { Longifude } \\
\text { (degrees, } \\
\text { minutes, } \\
\text { seconds) }\end{array}$ & $\begin{array}{c}\text { Elevation } \\
\text { (feet above } \\
\text { sea level) }\end{array}$ & $\begin{array}{l}\text { Date began } \\
\text { reporting }\end{array}$ \\
\hline MER & Mercury & W & $39^{\circ} 39^{\prime} 28^{\prime \prime}$ & $115^{\circ} 59^{\circ} 45^{\prime \prime}$ & 3,770 & $03-71$ \\
\hline RV & Rock Valley & $\mathrm{TB} / \mathrm{S}$ & $36^{\circ} 41^{\circ} 07^{\prime \prime}$ & $116^{\circ} 11^{\prime} 55^{\prime \prime}$ & 3,400 & $02-63$ \\
\hline $4 \mathrm{JA}$ & Jackass Flats & $\mathrm{TB} / \mathrm{S}$ & $36^{\circ} 47^{\circ} 05^{\circ}$ & $116^{\circ} 17^{\circ} 20^{\prime \prime}$ & 3,422 & $01-67$ \\
\hline DRA & Deser Rock & $\mathrm{S}$ & $36^{\circ} 37^{\prime} 26^{\prime \prime}$ & $115^{\circ} 59^{\prime} 18^{\prime \prime}$ & 3,298 & $10-63$ \\
\hline $\mathrm{CS}$ & Cane Spring & W & $36^{\circ} 48^{\prime} 43^{\prime \prime}$ & $116^{\circ} 06^{\prime} 28^{\prime \prime}$ & 4,000 & $09-64$ \\
\hline W5B & Well 5B & W & $36^{\circ} 48^{\circ} 07^{\prime \prime}$ & $115^{\circ} 57^{\circ} 52^{\prime \prime}$ & 3,080 & $09-63$ \\
\hline MV & Mid Valley & $\mathbb{W}$ & $36^{\circ} 58^{\prime} 20^{\circ}$ & $116^{\circ} 10^{\circ} 19^{\prime \prime}$ & 4,660 & $09-64$ \\
\hline $\mathrm{UCC}$ & Yucca Lake & $W$ & $36^{\circ} 57^{\prime} 23^{\prime \prime}$ & $116^{\circ} 02^{\prime} 51^{\prime \prime}$ & 3,924 & $05-58$ \\
\hline $40 \mathrm{MN}$ & Fortymile North & W & $37^{\circ} 02^{\prime} 57^{\prime \prime}$ & $116^{\circ} 17^{\prime} 16^{\prime \prime}$ & 4,820 & $02-60$ \\
\hline $\mathrm{TS} 2$ & Tippipah Springs & $W$ & $37^{\circ} 02^{\prime} 29^{\prime \prime}$ & $116^{\circ} 14^{\prime} 07^{\prime \prime}$ & 5,470 & $02-60$ \\
\hline BIY & Buster Jangle $\mathrm{Y}$ & W & $37^{\circ} 03^{\prime} 45^{\prime \prime}$ & $116^{\circ} 03^{\prime} 10^{\prime \prime}$ & 4,070 & $02-60$ \\
\hline $\mathrm{A} 12$ & Area 12 Mesa & W & $37^{\circ} 11^{\prime} 24^{\prime \prime}$ & $116^{\circ} 12^{\prime} 56^{\prime \prime}$ & 7.490 & $03-59$ \\
\hline PHS & $\begin{array}{l}\text { Public Health Service } \\
\text { Farm }\end{array}$ & $\mathrm{TB} / \mathrm{S}$ & $37^{\circ} 12^{\prime} 32^{\prime \prime}$ & $116^{\circ} 02^{\prime} 19^{\prime \prime}$ & 4,565 & $10-64$ \\
\hline PM1 & Pahute Mesa \#1 & $\mathrm{TB} / \mathrm{S}$ & $37^{\circ} 14^{\prime} 56^{\prime \prime}$ & $116^{\circ} 26^{\prime} 15^{\prime \prime}$ & 6,550 & $01-64$ \\
\hline $\mathrm{LF} 2$ & Little Feller $\$ 2$ & W & $37^{\circ} 07^{\prime} 05^{\prime \prime}$ & $116^{\circ} 18^{\prime} 13^{\prime \prime}$ & 5,120 & $08-76$ \\
\hline LFI & Little Feller 1 & $\mathrm{~TB}$ & $37^{\circ} 06^{\prime} 59^{\prime \prime}$ & $116^{\circ} 19^{\prime} 01^{\prime \prime}$ & 5,160 & $a_{08-76}$ \\
\hline PU13 & Plutonium 13 & $\mathrm{~TB}$ & $37^{\circ} 18^{\prime} 29^{\prime \prime}$ & $115^{\circ} 54^{\prime} 36^{\prime \prime}$ & 4,580 & $b_{01-72}$ \\
\hline
\end{tabular}

${ }^{a}$ Discontinued May $9,1984$.

$b$ Discontinued May $10,1984$. 
TABLE 7.--Precipitation data from Weather Service Nuclear Support Office network stations. All precipitation values in inches.

Station.-MER, Mercury; RV, Rock Valley; 4JA, Jackass Flats; DRA Desert Rock; CS, Cane Spring; W5B, Well 5B; MV, Mid Valley; UCC, Yucca Lake; 40MN, Fortymile North; TS2, Tippipah Springs; BJY, Buster Jangle Y; A12, Area 12 Mesa; PHS, Public 1 tealth Service Farm; PM1, Pahute Mesa 1 1; LF2, Little Feller \#2; LF1, Little Feller \#1; PU13, Plutonium 13.

\begin{tabular}{|c|c|c|c|c|c|c|c|c|c|c|c|c|c|c|c|c|c|}
\hline \multirow[b]{2}{*}{ DAY } & \multicolumn{17}{|c|}{ Station } \\
\hline & MER & RV & $4 J \mathrm{~A}$ & DRA & $\mathrm{CS}$ & W5B & MV & $\mathrm{UCC}$ & $40 \mathrm{MN}$ & TS2 & BJY & $\mathrm{A} 12$ & PHS & PM1 & $\mathrm{LF}_{2}$ & LF1 & PU13 \\
\hline \multicolumn{18}{|c|}{ OCTOBER 1982} \\
\hline 1 & -- & -- & -- & -- & -- & -- & -- & -- & -- & - & -- & -. & - & - & -- & - & $(a)$ \\
\hline 2 & -- & -- & -- & -- & - & - & $\ldots$ & $\ldots$ & $\infty$ & $\ldots$ & $\cdots$ & $\infty$ & $\infty$ & $\cdots$ & $\ldots$ & -. & $(a)$ \\
\hline 3 & -- & -- & - & -- & - & -- & $-\infty$ & -- & -- & - & -- & -- & - & - & -- & -- & (a) \\
\hline 4 & $-\infty$ & -- & -- & - & -- & $-\infty$ & $\infty$ & - & - & $-\infty$ & - & -- & - & - & $\infty$ & $\ldots$ & (a) \\
\hline 5 & -- & -- & - & -- & -- & -- & -- & $-\infty$ & - & - & - & - & - & - & - & - & $(a)$ \\
\hline 6 & - & $\ldots$ & - & -- & -- & -- & -- & -- & - & - & -- & $\ldots$ & -. & - & -- & -- & (a) \\
\hline 7 & $\ldots$ & $\ldots$ & -- & $\ldots$ & -- & $\ldots$ & -- & $-\infty$ & -- & $\infty$ & $\ldots$ & - & $\ldots$ & $\ldots$ & .. & .. & (a) \\
\hline 8 & -- & -- & - & - & - & -- & $\ldots$ & -- & $=$ & -- & -- & -- & -- & - & -. & -- & $(a)$ \\
\hline 9 & -- & -- & - & -- & -- & -. & -- & -- & $\infty$ & - & $\ldots$ & -- & $\ldots$ & $\infty$ & $\ldots$ & $\ldots$ & (aI) \\
\hline 10 & - & -- & -- & -- & -- & -- & - & $\cdots$ & -- & - & - & -- & -- & -- & $\cdots$ & -- & (a) \\
\hline 11 & -- & $\ldots$ & - & - & - & -- & -- & -- & $\ldots$ & -. & -- & -- & -- & $\ldots$ & $\infty$ & $\ldots$ & $\{a\}$ \\
\hline 12 & $\ldots$ & - & -- & -- & -- & -- & -- & - & $\infty$ & -- & $\ldots$ & $\ldots$ & -- & -. & $\ldots$ & -. & (a) \\
\hline 13 & -- & -- & -- & -- & -- & -- & $\ldots$ & -- & - & -- & -- & -- & -- & -. & $\ldots$ & -. & - \\
\hline 14 & -. & -- & $\ldots$ & $\ldots$ & $\infty$ & $\ldots$ & $\ldots$ & -. & -- & -- & -- & -- & $\ldots$ & $\ldots$ & . & -. & -- \\
\hline 15 & -- & -- & - & - & -- & +- & -. & -. & $=$ & - & 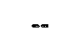 & -- & - & - & -. & -- & - \\
\hline 16 & - & -- & -- & -- & -- & -- & -- & -- & - & -- & $-\infty$ & -. & - & -. & -. & -. & $\ldots$ \\
\hline 17 & -- & -- & -. & -- & -. & $\ldots$ & . & -. & $-\infty$ & -- & $\ldots$ & -. & - & (a) & $\infty$ & -. & - \\
\hline 18 & -. & -- & -- & -- & -- & -. & -. & -- & $\ldots$ & -- & -- & -- & -. & (a) & $\ldots$ & $\ldots$ & - \\
\hline 19 & -- & - & - & - & -. & -. & $\ldots$ & -. & - & - & -. & -. & - & (a) & $\ldots$ & $\ldots$ & - \\
\hline 20 & 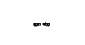 & - & - & - & -- & -- & -- & -- & - & -- & .. & -. & -- & $(a)$ & -. & -- & $\ldots$ \\
\hline 21 & -- & -- & -. & -- & -- & - & -- & -. & - & -. & -. & -- & -. & $(a)$ & $\ldots$ & $\ldots$ & -- \\
\hline 22 & -- & -- & $\ldots$ & - & - & 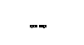 & $\cdots$ & -- & $\ldots$ & $=$ & $=$ & $\infty$ & -- & (a) & - & -- & $\ldots$ \\
\hline 23 & -- & -. & - & -- & - & -- & -- & -- & -. & -- & -- & $\ldots$ & .. & (a) & -. & -. & -- \\
\hline 24 & 0.02 & 0.01 & 0.01 & 0.02 & 0.02 & 0.02 & 0.02 & 0.04 & $\ldots$ & 0.03 & 0.02 & 0.18 & 0.05 & (b) & 0.03 & 0.02 & 0.02 \\
\hline 25 & -- & -- & - & -- & - & -- & -- & - & -. & - & -- & $\ldots$ & $-\infty$ & (b) & $\ldots$ & -- & -- \\
\hline 26 & -- & -. & -- & (c) & - & -- & .07 & .02 & 0.03 & .22 & - & .14 & -- & $d_{0.40}$ & .06 & .05 & (a) \\
\hline 27 & - & $\ldots$ & - & $\ldots$ & -. & $\ldots$ & - & - & - & -- & - & .14 & -. & (a) & - & .. & $(a)$ \\
\hline 28 & $=$ & $=$ & $=$ & - & $-\infty$ & $=$ & -. & -. & -- & -- & - & $m$ & - & (a) & $=$ & $\infty$ & (a) \\
\hline 29 & -- & -- & -- & -- & -- & -- & -- & -- & -- & -- & -- & $\ldots$ & -- & (a) & -- & -- & (a) \\
\hline 30 & .23 & .13 & - & .31 & .09 & .18 & .08 & .08 & -- & .07 & .05 & .19 & .09 & (b) & .05 & .04 & (b) \\
\hline 31 & .01 & - & .01 & .02 & .02 & .01 & .. & .01 & .02 & .02 & .03 & .03 & .02 & $d_{.25}$ & $\ldots$ & .02 & $d .30$ \\
\hline Total & 0.26 & 0.14 & 0.02 & 0.35 & 0.13 & 0.21 & 0.17 & 0.15 & 0.05 & 0.34 & 0.10 & 0.54 & 0.16 & $e_{0.65}$ & 0.14 & 0.13 & 0.32 \\
\hline
\end{tabular}

See page 66 for explanation of footnotes. 
TABLE 7.--Preciptation data from Weather Service Nuclear Support Office nework stations--Continued.

\begin{tabular}{|c|c|c|c|c|c|c|c|c|c|c|c|c|c|c|c|c|c|}
\hline \multirow[b]{2}{*}{ DAY } & \multicolumn{17}{|c|}{ Station } \\
\hline & MER & RV & $4 \mathrm{IA}$ & DRA & $\mathrm{CS}$ & W5B & $\mathrm{MV}$ & $\mathrm{UCC}$ & $40 \mathrm{MN}$ & $\mathrm{T} \$ 2$ & BJY & A12 & PHS & PM1 & LF2 & LF1 & PU13 \\
\hline \multicolumn{18}{|c|}{ NOVEMBER 1982} \\
\hline $\mathbb{1}$ & 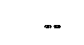 & $-\infty$ & $-\infty$ & - & -- & -- & -- & -- & -- & -. & $\infty$ & -- & - & (a) & -- & -- & -- \\
\hline 2 & - & -- & -- & -- & -- & -- & -- & -- & -- & -. & - & -- & -- & (a) & - & - & -- \\
\hline 3 & -- & -- & -- & -- & -. & $-\infty$ & - & - & - & - & - & -- & -- & $(a)$ & - & - & - \\
\hline 4 & - & -- & -- & $\infty$ & 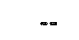 & $-\infty$ & $-\infty$ & $\infty$ & $-\infty$ & -- & -- & - & -- & (a) & - & $-\infty$ & -- \\
\hline 5 & - & - & $-\cdots$ & $\cdots$ & - & -- & -- & - & - & -- & -- & -- & -- & (a) & -- & -- & -- \\
\hline 6 & $\infty$ & -. & $\ldots$ & -- & -- & -. & -- & $-\infty$ & .. & -. & $\infty$ & $\infty$ & $\ldots$ & (a) & - & -- & -- \\
\hline 7 & -- & -- & -- & -- & -- & -- & -- & -- & -- & -. & -- & -- & -- & (a) & -- & -- & -- \\
\hline 8 & - & - & .. & -. & - & -. & -- & .. & -. & -- & -. & -- & -- & (a) & .. & -. & -- \\
\hline 9 & 0.42 & 0.47 & 0.28 & 0.42 & 0.57 & 0.29 & 0.29 & 0.38 & 0.25 & 0.30 & 0.37 & 0.58 & 0.18 & (b) & (b) & 0.05 & 0.09 \\
\hline 16 & .38 & .31 & .21 & .21 & .32 & .12 & .38 & .19 & .29 & .38 & .15 & .22 & .39 & .30 & $f .50$ & .37 & (b) \\
\hline 11 & $\ldots$ & - & $\ldots$ & - & $-\infty$ & -- & -- & -- & -- & - & -- & -- & $=$ & (a) & -. & -. & (b) \\
\hline 19 & $\cdots$ & $\cdots$ & $\ldots$ & $\cdots$ & - & - & -- & -. & -- & -- & - & -- & - & (a) & -- & -- & (b) \\
\hline 13 & - & -. & -- & - & -- & - & -- & -- & - & $\infty$ & - & -- & -- & (a) & $-\infty$ & $-\infty$ & (b) \\
\hline 14 & - & -. & -. & -- & -- & - & -- & -- & -- & -- & -- & -- & -. & (a) & - & -- & (b) \\
\hline 15 & - & -. & - & - & -- & -- & -. & -- & -- & -- & -- & -- & -- & (a) & -- & -- & (b) \\
\hline 16 & $m$ & $=$ & -. & - & - & -. & -. & - & .- & $\infty$ & $-\infty$ & -- & - & $(a)$ & - & .. & (b) \\
\hline 17 & -- & -- & -- & - & - & -- & -- & -. & -. & - & -- & -. & -- & (a) & -- & - & (b) \\
\hline 18 & - & -. & - & - & - & - & -- & -. & -- & -- & $\ldots$ & - & - & (a) & $\ldots$ & -. & (b) \\
\hline 19 & $=$ & $\infty$ & $=$ & $\infty$ & $-\infty$ & -- & -- & $-\infty$ & - & - & .03 & -- & -- & (a) & -- & -- & (b) \\
\hline 20 & -- & - & - & -- & - & - & -- & -- & -- & -- & -- & -- & - & (a) & -- & -- & (b) \\
\hline 21 & - & - & -- & - & -- & -- & $=$ & -. & $\ldots$ & -- & - & -- & -- & (a) & $\infty$ & $-\infty$ & (b) \\
\hline 22 & .02 & .01 & .01 & .02 & .04 & .02 & .19 & .18 & .14 & .26 & .23 & .27 & .18 & (b) & .16 & (b) & (b) \\
\hline 23 & .01 & - & .01 & (c) & - & .01 & .06 & .01 & .04 & .09 & .05 & .11 & .01 & ${ }^{d} .15$ & .10 & $f .26$ & (b) \\
\hline 21 & $\ldots$ & -. & - & - & -- & -- & -- & -- & - & -. & -- & .- & - & (a) & -- & -- & (b) \\
\hline 35 & - & - & - & - & -- & -- & - & -- & - & -. & $\ldots$ & $=$ & 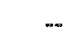 & (a) & -- & -- & (b) \\
\hline 26 & $\ldots$ & - & - & - & -- & - & - & $=$ & $-\infty$ & - & -- & -- & $\ldots$ & $(a)$ & -- & -- & (b) \\
\hline 27 & - & - & -- & -- & -- & -- & -- & - & -- & -- & - & -- & -- & $(a)$ & -- & -. & (b) \\
\hline 28 & -- & -- & -- & -- & -- & 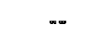 & $-\infty$ & - & $\ldots$ & -. & $=$ & -- & $-\infty$ & $(a)$ & .. & - & (b) \\
\hline 29 & -- & - & - & -. & -. & -- & -- & -- & -- & -- & -- & -. & -- & (a) & -. & -- & (b) \\
\hline 30 & .25 & .18 & .15 & .25 & .46 & .18 & 1.40 & .48 & .88 & 1.02 & .39 & 1.13 & .38 & ${ }^{d} .40$ & .61 & .57 & 8.80 \\
\hline Tral & 1.08 & 0.97 & 0.66 & 0.90 & 1.39 & 0.62 & 2.32 & 1.24 & 1.60 & 2.05 & 1.22 & 2.31 & 1.14 & $e_{0.85}$ & 1.37 & 1.25 & ${ }^{e} 0.89$ \\
\hline
\end{tabular}


TABLE 7.--Precipitation data from Weather Service Nuclear Support Office network stations--Continued.

\begin{tabular}{|c|c|c|c|c|c|c|c|c|c|c|c|c|c|c|c|c|c|}
\hline \multirow[b]{2}{*}{ DAY } & \multicolumn{17}{|c|}{ Station } \\
\hline & MER & RV & 4JA & DRA & $\mathrm{CS}$ & W5B & MV & UCC & $40 \mathrm{MN}$ & TS2 & BIY & A12 & PHS & PM1 & $\mathrm{LF} 2$ & LF1 & PU13 \\
\hline \multicolumn{18}{|c|}{ DECEMBER 1982} \\
\hline 1 & - & (a) & - & - & -- & - & - & - & -- & -- & -- & -- & -- & (a) & - & -- & (a) \\
\hline 2 & -- & (a) & -- & -- & -- & -- & -- & -- & $\ldots$ & -- & $=$ & -- & -. & (a) & $\ldots$ & .. & (a) \\
\hline 3 & -- & $(a)$ & -- & $\ldots$ & -- & -- & - & - & -- & -- & - & -- & -. & (a) & -- & -- & (a) \\
\hline 4 & $\infty$ & (a) & -- & - & -- & - & $-\infty$ & -- & -- & -- & - & -- & -. & (a) & -- & - & (a) \\
\hline 5 & -- & $(a)$ & -- & -- & -- & -- & -- & -- & - & -- & - & $-\infty$ & -- & (a) & -- & - & (a) \\
\hline 6 & - & $(a)$ & -. & -- & -. & -- & -- & - & -- & -- & - & -- & -- & (a) & -- & -- & (a) \\
\hline 7 & -- & -- & -- & -- & -- & -- & - & -- & - & -- & $=$ & -- & - & (a) & -. & -- & (a) \\
\hline 8 & 0.05 & -.. & -- & 0.05 & -. & -- & -- & .. & -- & -- & - & 0.01 & -- & (a) & -. & -- & (b) \\
\hline 9 & .01 & 0.06 & 0.04 & .01 & 0.14 & 0.09 & 0.23 & 0.13 & 0.15 & 0.21 & 0.14 & .56 & 0.16 & 0.02 & 0.02 & 0.02 & (b) \\
\hline 10 & - & (a) & -- & -- & -- & - & -- & - & -- & -. & -- & .04 & -- & -- & -- & - & (b) \\
\hline 11 & - & (a) & -- & -- & $\cdots$ & -- & -- & -- & - & -- & -- & - & -- & -- & -- & -- & (b) \\
\hline 12 & -- & (a) & - & -- & -- & -- & -- & -- & -. & -- & - & -- & -- & -- & -- & -- & (b) \\
\hline 13 & .05 & (a) & .01 & .04 & .06 & .04 & .03 & - & .02 & .03 & - & .05 & - & -- & .02 & -- & (b) \\
\hline 14 & - & - & -- & - & -- & -- & -- & -- & - & -- & -- & -- & -- & -- & -- & - & (b) \\
\hline 15 & $\cdots$ & - & -- & - & -. & -- & - & -- & -- & -- & - & -- & -- & -- & - & - & (b) \\
\hline 16 & -- & -- & $=$ & $-\infty$ & -. & -- & - & -- & -- & - & - & -- & - & - & -- & -- & (b) \\
\hline 17 & - & -- & -- & -- & - & -- & - & -- & -- & -- & -- & - & - & -- & -- & -- & (b) \\
\hline 18 & - & -- & -- & -- & -- & -- & -- & -- & -- & -- & - & -- & - & -- & -. & - & (b) \\
\hline 19 & -- & -- & - & -- & -- & $-\infty$ & - & -- & -. & -- & - & - & -- & -- & - & - & (b) \\
\hline 20 & - & -- & - & -- & -- & - & - & -- & -- & -- & -- & -- & -- & - & - & - & (b) \\
\hline 21 & -- & -- & -- & $\cdots$ & -- & -- & -- & .. & -. & -- & - & -. & -. & .. & -- & - & (b) \\
\hline 22 & .14 & .30 & .18 & .19 & .45 & .17 & .59 & .18 & .29 & .39 & .19 & (b) & .22 & .09 & .28 & .30 & (b) \\
\hline 23 & .02 & .05 & .02 & .03 & .03 & .01 & .05 & .02 & .09 & .04 & .02 & $f .64$ & .04 & -. & .06 & .01 & 0.20 \\
\hline 24 & - & -- & - & - & - & - & -- & -. & -- & -- & -- & (a) & -- & -- & - & $=$ & (a) \\
\hline 25 & - & - & -- & -- & - & -- & -- & -- & - & -- & - & (a) & -- & -- & -- & -- & (a) \\
\hline 26 & - & - & $\cdots$ & -- & -- & - & -- & -- & -- & $\cdots$ & -- & (a) & - & -- & -- & -- & (a) \\
\hline 27 & - & - & - & - & -. & -- & -- & -. & - & -- & - & (a) & -- &.- & - & - & (a) \\
\hline 28 & -- & - & - & -- & -- & -- & -- & -- & -- & -- & - & (a) & - & -. & -- & - & (a) \\
\hline 29 & $-\infty$ & $\ldots$ & -. & - & -. & -- & -- & -- & $\infty$ & -- & -. & (a) & -- & - & - & - & $(a)$ \\
\hline 30 & -- & - & -- & -- & -. & -- & -- & -- & -- & -- & -- & -- & -- & -- & - & -- & (a) \\
\hline 31 & - & $-\infty$ & -- & -- & -. & -- & -- & -- & -- & - & -- & - & - & -- & -- & -- & (a) \\
\hline Total & 0.27 & 0.41 & 0.25 & 0.32 & 0.68 & 0.31 & 0.90 & 0.33 & 0.55 & 0.67 & 0.35 & 1.30 & 0.42 & 0.11 & 0.38 & 0.33 & ${ }^{e} 0.20$ \\
\hline
\end{tabular}


TABLE 7.--Precipitation data from Weather Service Nuclear Support Office network stations--Continued.

\begin{tabular}{|c|c|c|c|c|c|c|c|c|c|c|c|c|c|c|c|c|c|}
\hline \multirow[b]{2}{*}{ DAY } & \multicolumn{17}{|c|}{ Station } \\
\hline & MER & RV & $4 J A$ & DRA & $\mathrm{CS}$ & W5B & MV & UCC & $40 \mathrm{MN}$ & $\mathrm{TS} 2$ & BJY & $\mathrm{A} 12$ & PHS & PM1 & $\mathrm{LF}_{2}$ & LF1 & PU13 \\
\hline \multicolumn{18}{|c|}{ JANUARY 1983} \\
\hline 1 & -- & -- & $\infty$ & $\cdots$ & - & -- & - & $-\infty$ & -- & -- & - & -- & - & (a) & -- & - & - \\
\hline 2 & -- & -- & -- & -- & - & - & -- & -- & - & -- & -- & -- & -- & (a) & - & - & - \\
\hline 3 & -. & -- & - & -- & -- & - & -- & -- & -- & - & -- & -- & - & (a) & - & - & -. \\
\hline 4 & $-\infty$ & $-\infty$ & $-\cdots$ & -. & $-\infty$ & -- & $-\infty$ & $\ldots$ & $-\infty$ & -- & -- & -- & -- & (a) & - & - & $\ldots$ \\
\hline 5 & -- & -- & -- & - & -- & -- & -- & -- & -- & -- & -- & -- & - & (a) & -- & - & - \\
\hline 6 & -- & -. & -- & $\ldots$ & -. & -- & -. & -- & -- & -- & - & - & $-\infty$ & (a) & -- & - & $\ldots$ \\
\hline 7 & - & -- & -- & -- & - & -- & - & - & - & -. & -- & -- & - & $(a)$ & - & -- & - \\
\hline 8 & -. & -- & - & -- & -- & $-\infty$ & -- & $\ldots$ & -- & -- & -. & -- & -- & (a) & -- & - & -. \\
\hline 9 & -. & -- & $\ldots$ & - & - & -- & $=$ & -- & -. & - & - & $-\infty$ & -- & (a) & -- & - & - \\
\hline 10 & -- & -- & -- & -- & -- & -- & - & - & -- & -- & -- & -- & - & (a) & - & - & - \\
\hline 11 & $=$ & -- & - & -- & -- & - & -- & -- & $\infty$ & $-\infty$ & -. & - & - & (a) & $-\infty$ & $-\infty$ & $-\infty$ \\
\hline 12 & -- & -. & -- & -- & -- & -- & -- & -- & -- & -- & -- & -- & -- & (a) & -- & - & - \\
\hline 13 & -- & -- & -. & $\ldots$ & - & -- & $\infty$ & -- & .- & .. & -- & -. & - & (a) & -. & - & - \\
\hline 14 & - & -. & -. & -- & -- & - & - & -. & -. & -- & .- & - & .. & (a) & -- & -- & -- \\
\hline 15 & -- & -- & -- & -- & -- & - & -- & -- & -. & -- & -- & - & -- & (a) & -- & -- & - \\
\hline 16 & 0.15 & 0.04 & 0.05 & 0.11 & 0.22 & 0.14 & 0.17 & 0.15 & 0.12 & 0.48 & 0.15 & 0.32 & 0.21 & (b) & 0.27 & 0.22 & 0.21 \\
\hline 17 & .01 & .01 & .01 & -- & .03 & .03 & .03 & .02 & .10 & .09 & .12 & .07 & .05 & (b) & .14 & .12 & .01 \\
\hline 18 & -- & -- & -- & -. & - & -- & - & - & -- & -- & - & -- & - & (b) & - & - & - \\
\hline 19 & .03 & .16 & .07 & .03 & .08 & .02 & .19 & .10 & .05 & .34 & .16 & .41 & .13 & (b) & .20 & .13 & .11 \\
\hline 20 & -- & -- & -- & - & - & -- & - & -. & -- & -- & -- & -- & -- & (b) & -- & - & - \\
\hline 21 & -. & - & -- & -. & -. & -- & - & -- & -- & -- & -- & -- & -- & (b) & - & -- & - \\
\hline 22 & .15 & .12 & .10 & .14 & .16 & .12 & .13 & .11 & .04 & .15 & .12 & .13 & .01 & (b) & .07 & .03 & - \\
\hline 23 & .03 & .03 & .03 & .11 & .05 & .02 & - & .05 & .05 & .02 & .04 & .03 & .07 & (b) & .05 & .10 & .11 \\
\hline 24 & .04 & .01 & .28 & .07 & .72 & .22 & .47 & .36 & .21 & .30 & .23 & .34 & .06 & (b) & .16 & .06 & .19 \\
\hline 25 & -- & -- & -- & -- & - & - & - & - & - & -- & -- & $\ldots$ & - & (b) & -- & - & (a) \\
\hline 26 & -- & -- & -. & -- & -. & - & -- & - & -- & -- & 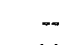 & - & -- & (b) & - & - & (a) \\
\hline 27 & .11 & .25 & .13 & .14 & .36 & .14 & .70 & .47 & .50 & .44 & .44 & .53 & .41 & (b) & .40 & .32 & (b) \\
\hline 28 & 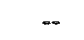 & $\infty$ & .. & -. & -- & - & - & $\infty$ & -- & -. & -- & $\infty$ & -- & (b) & -- & - & (b) \\
\hline 29 & .12 & .54 & .56 & .16 & .58 & .27 & .65 & .57 & .61 & .67 & .46 & 1.11 & .55 & ${ }^{d} 1.15$ & .47 & .19 & ${ }^{d} .60$ \\
\hline 30 & -- & -- & -- & -- & -- & - & - & $\infty$ & - & -- & - & $-\infty$ & -- & (a) & -- & -- & (a) \\
\hline 31 & -- & -- & -- & -- & $-\infty$ & -- & -- & - & $\cdots$ & $\ldots$ & -- & -- & - & (a) & - & - & (a) \\
\hline Total & 0.64 & 1.16 & 1.23 & 0.76 & 2.20 & 0.96 & 2.34 & 1.83 & 1.68 & 2.49 & 1.72 & 2.94 & 1.49 & $e_{1.15}$ & 1.76 & 1.17 & ${ }^{e} 1.23$ \\
\hline
\end{tabular}


TABLE 7.--Precipitation data from Weather Service Nuclear Support Office network stations--Continued.

\begin{tabular}{|c|c|c|c|c|c|c|c|c|c|c|c|c|c|c|c|c|c|}
\hline \multirow[b]{2}{*}{ DAY } & \multicolumn{17}{|c|}{ Station } \\
\hline & MER & RV & $4 \mathrm{JA}$ & DRA & $\mathrm{CS}$ & W5B & MV & UCC & $40 \mathrm{MN}$ & TS2 & BJY & $\mathrm{A} 12$ & PHS & PM1 & $\mathrm{LF}_{2}$ & $\mathrm{LF} 1$ & PU13 \\
\hline \multicolumn{18}{|c|}{ FEBRUARY 1983} \\
\hline 1 & -- & -- & -- & -- & -- & (a) & -- & -- & -- & -- & -- & - & -- & (a) & - & (a) & (a) \\
\hline 2 & -- & -- & -- & (c) & 0.02 & (b) & 0.02 & -- & -. & 0.03 & -- & 0.05 & -- & (b) & - & (b) & (a) \\
\hline 3 & - & -- & -- & 0.04 & .02 & (b) & .10 & 0.01 & 0.04 & .04 & $=$ & .15 & -- & (b) & - & (b) & -- \\
\hline 4 & -- & - & -- & - & - & (b) & -- & -- & -- & -- & -- & (a) & -- & (b) & -- & (b) & -- \\
\hline 5 & 0.07 & 0.15 & 0.18 & .15 & .14 & (b) & .10 & .10 & - & .10 & 0.07 & (b) & -- & (b) & 0.06 & $f_{0.05}$ & - \\
\hline 6 & .13 & .12 & .06 & .18 & .20 & (b) & .17 & .20 & .15 & .11 & .20 & (b) & 0.16 & (b) & .11 & .10 & 0.12 \\
\hline 7 & .04 & -- & .02 & (c) & .05 & $f_{0.08}$ & .15 & .07 & .12 & .09 & .08 & (b) & .08 & (b) & .11 & .07 & .09 \\
\hline 8 & -- & -- & -- & .01 & -- & $(a)$ & .17 & -- & .18 & .22 & .04 & (b) & .33 & (b) & .19 & .03 & .24 \\
\hline 9 & -- & -- & -- & - & - & (a) & (a) & - & -- & -- & -- & (b) & -- & (b) & -- & -- & -- \\
\hline 10 & -- & - & -- & - & -- & (a) & - & -. & -. & - & -. & (b) & $=$ & (b) & - & - & - \\
\hline 11 & -- & $-\infty$ & -- & -- & -- & (a) & -- & - & -- & -- & -- & (b) & - & (b) & - & -- & - \\
\hline 12 & -- & - & -- & - & -- & (a) & (a) & -. & -- & -- & -- & (b) & -- & (b) & -- & -- & -- \\
\hline 13 & .10 & .09 & .02 & .13 & .12 & .02 & .07 & .11 & .08 & .13 & .02 & (b) & .21 & (b) & .10 & .09 & .15 \\
\hline 14 & -- & -- & -- & -- & - & (a) & (a) & - & -- & -- & -- & (b) & -- & (b) & -- & -- & -- \\
\hline 15 & -. & -- & -- & -- & -- & -- & (a) & -- & - & -- & -- & (b) & -- & (b) & -- & - & -- \\
\hline 16 & $=$ & - & -- & - & -- & - & -. & -- & -- & -. & -- & (b) & $m$ & (b) & $-\infty$ & -- & -- \\
\hline 17 & -- & -- & -- & -- & -- & -- & -. & $\ldots$ & - & .. & -- & (b) & -. & (b) & -- & -- & -- \\
\hline 18 & -. & -- & $-\infty$ & (c) & - & -- & .04 & -- & -. & .02 & .02 & (b) & .06 & (b) & .09 & .08 & .06 \\
\hline 19 & -- & - & -- & -- & -- & - & -- & -- & -- & -- & -- & (b) & -- & (b) & -- & -. & -- \\
\hline 20 & - & -- & -- & -- & -- & -- & - & -- & -. & -- & -- & (b) & - & (b) & -- & - & -- \\
\hline 21 & -. & -- & - & -- & - & -- & -. & - & -. & -. & -- & (b) & -- & (b) & - & - & -- \\
\hline 22 & - & -- & -- & - & -. & - & -- & .. & -- & .- & .. & (b) & -. & (b) & - & - & -. \\
\hline 23 & -. & - & -. & -- & -- & -- & - & -- & -. & -- & .. & (b) & -- & (b) & -- & $\infty$ & -- \\
\hline 24 & .02 & .04 & .04 & .03 & .01 & .01 & -- & .03 & -. & .04 & .01 & (b) & .07 & (b) & .06 & .04 & (b) \\
\hline 25 & .03 & .01 & .01 & .03 & .03 & .02 & .02 & -- & .02 & .03 & -- & (b) & .01 & (b) & .04 & .01 & $f_{.05}$ \\
\hline 26 & -- & -- & -- & - & - & -- & .03 & -. & .03 & - & .01 & (b) & - & (b) & .03 & .01 & -- \\
\hline 27 & .11 & .05 & .03 & .11 & .24 & -- & .79 & .05 & .32 & .40 & .13 & (b) & .32 & (b) & .28 & .23 & .40 \\
\hline 28 & - & $=\infty$ & -- & - & -- & - & .01 & - & .03 & .03 & -- & $d_{2.60}$ & .01 & $d_{1.10}$ & .10 & .07 & -- \\
\hline Total & 0.50 & 0.46 & 0.36 & 0.68 & 0.83 & 0.13 & 1.67 & 0.57 & 0.97 & 1.24 & 0.58 & $e_{2.80}$ & 1.25 & $e_{1.10}$ & 1.17 & 0.78 & 1.11 \\
\hline
\end{tabular}


TABLE 7.--Precipitation data from Weather Service Nuclear Support Office nemork stations--Continued.

\begin{tabular}{|c|c|c|c|c|c|c|c|c|c|c|c|c|c|c|c|c|c|}
\hline \multirow[b]{2}{*}{ DAY } & \multicolumn{17}{|c|}{ Station } \\
\hline & MER & RV & $4 J \mathrm{~A}$ & DRA & $\mathrm{CS}$ & W5B & MV & $\mathrm{UCC}$ & $40 \mathrm{MN}$ & $\mathrm{TS} 2$ & B.JY & $\mathrm{A} 12$ & PHS & PM1 & LF2 & $\mathrm{LF} \mathbb{1}$ & PU13 \\
\hline \multicolumn{18}{|c|}{ MARCH 1983} \\
\hline 1 & 0.24 & 0.62 & 0.55 & 0.46 & 0.55 & 0.21 & 0.78 & 0.47 & 1.31 & 1.18 & 0.56 & (b) & 0.75 & (b) & (b) & 0.76 & (b) \\
\hline 2 & .23 & .66 & .60 & .35 & .82 & .17 & .76 & .64 & .97 & 1.19 & .50 & (b) & .34 & (b) & (b) & .62 & (b) \\
\hline 3 & .22 & .52 & .73 & .35 & .67 & - & .94 & .56 & .98 & 1.02 & .68 & (b) & .56 & (b) & $\hat{f}_{2.63}$ & .13 & $f .80$ \\
\hline 4 & .03 & .11 & .01 & .20 & -- & -- & -- & -- & - & -. & .02 & $d_{4.95}$ & - & $d_{2.10}$ & -. & -. & (a) \\
\hline 5 & -- & -- & - & -- & -- & - & -- & -- & -- & -- & -- & (a) & -- & $(a)$ & - & - & (a) \\
\hline 6 & .- & -- & .- & .. & -- & - & -- & -. & $\ldots$ & -. & .- & (a) & -. & (a) & $\ldots$ & .. & (a) \\
\hline 7 & -- & -- & .. & -- & -- & - & -- & - & -- & -- & $\ldots$ & (a) & - & (a) & -. & -. & (a) \\
\hline 8 & -- & -. & -- & -- & - & - & -- & -- & -- & -- & -- & (a) & -- & (a) & - & - & (a) \\
\hline 9 & -- & .. & .. & -- & -- & - & -. & - & -- & $-\infty$ & -- & -. & - & (a) & -- & $\ldots$ & (a) \\
\hline 10 & -. & -- & -. & -- & -- & -- & -- & -- & -- & - & -. & -- & -- & (a) & -- & -. & -- \\
\hline 11 & -- & - & -- & -. & -- & -- & - & - & -. & -- & - & -- & -- & (a) & -- & $\ldots$ & -- \\
\hline 12 & .- & -. & $=$ & .. & -- & -- & -- & -- & (a) & -- & -- & (a) & -. & (a) & -. & $\ldots$ & -- \\
\hline 13 & -- & -- & -. & (c) & -- & $\ldots$ & -. & -- & (a) & -- & -- & (a) & -- & (a) & -- & -. & $\ldots$ \\
\hline 14 & -- & -. & .. & - & - & - & -- & -. & (a) & -- & - & (a) & -. & (a) & -- & -. & $-\infty$ \\
\hline 15 & - & -- & -- & -- & -- & -- & - & -- & (a) & - & -- & (a) & -- & (a) & -- & -. & -- \\
\hline 16 & -- & -- & -- & -. & -. & - & -. & -- & (a) & - & -- & (a) & .. & (a) & $\ldots$ & .. & -. \\
\hline 17 & .03 & .. & -- & .12 & .05 & .05 & .02 & .01 & (b) & .06 & .01 & .25 & (b) & (b) & .08 & .03 & -. \\
\hline 18 & .10 & .14 & .33 & .14 & .40 & .08 & .47 & .34 & (b) & .37 & .42 & (b) & (b) & (b) & .32 & .32 & .35 \\
\hline 19 & -- & - & $\ldots$ & - & - & $=$ & - & - & (b) & - & $\ldots$ & (b) & (b) & (b) & $=$ & $\ldots$ & $\ldots$ \\
\hline 20 & -- & -- & -. & -- & -- & -- & - & -- & (b) & - & -- & (b) & (b) & (b) & -- & .- & -- \\
\hline 21 & .48 & .66 & .78 & .56 & .64 & .34 & .62 & .44 & (b) & .62 & .50 & (b) & (b) & (b) & .58 & .42 & .25 \\
\hline 22 & -- & -- & -. & -. & -- & - & -- & -- & (b) & - & -- & (b) & (b) & (b) & - & -- & -- \\
\hline 23 & -- & -- & $\ldots$ & -- & -- & -- & .01 & -- & (b) & .02 & .02 & (b) & (b) & (b) & -- & - & .. \\
\hline 24 & -. & .14 & - & .01 & .12 & .04 & .32 & .08 & (b) & .13 & .04 & (b) & (b) & (b) & .10 & .06 & (b) \\
\hline 25 & -- & .01 & -- & (c) & -- & -- & - & -. & (b) & -- & - & (b) & (b) & ${ }^{d} .75$ & -- & - & (b) \\
\hline 26 & -- & -. & -. & .- & -. & - & $=$ & -- & (b) & -- & $\ldots$ & (b) & (b) & -- & -. & -. & (b) \\
\hline 27 & -- & -- & - & -- & -. & -- & -- & -- & (b) & - & -. & (b) & (b) & -- & - & -- & (b) \\
\hline 28 & -- & -- & -. & -- & -- & -- & .07 & -- & $f_{1.44}$ & .03 & -- & $g_{2.50}$ & $f_{1.45}$ & .18 & -- & -- & $f .30$ \\
\hline 29 & $=$ & -- & -- & - & -- & -- & - & -- & (a) & - & - & $(a)$ & $(a)$ & - & -- & -- & (a) \\
\hline 30 & -- & -- & - & -- & -- & -- & -- & -. & (a) & -- & -- & (a) & (a) & - & -- & - & (a) \\
\hline 31 & $\ldots$ & - & $\ldots$ & $=-$ & -- & $-\infty$ & - & -- & (a) & -- & $\ldots$ & (a) & (a) & - & - & $\ldots$ & (a) \\
\hline Total & 1.33 & 2.86 & 3.00 & 2.19 & 3.25 & 0.89 & 3.99 & 2.54 & 4.70 & 4.62 & 2.75 & $e_{7.70}$ & 3.10 & $e_{3.03}$ & 3.71 & 2.34 & 1.70 \\
\hline
\end{tabular}


TABLE 7.--Precipitation data from Weather Service Nuclear Support Office network stations--Continued.

\begin{tabular}{|c|c|c|c|c|c|c|c|c|c|c|c|c|c|c|c|c|c|}
\hline \multirow[b]{2}{*}{ DAY } & \multicolumn{17}{|c|}{ Station } \\
\hline & MER & RV & $4 J A$ & DRA & CS & W $5 B$ & $3 \mathrm{MV}$ & $\mathrm{UCC}$ & $40 \mathrm{MN}$ & $\mathbb{T S} 2$ & BJY & A12 & PHS & PM1 & $\mathrm{LF} 2$ & LFI & PU13 \\
\hline \multicolumn{18}{|c|}{ APRIL 1983} \\
\hline 1 & - & -- & - & -- & -- & -- & - & -- & $(a)$ & -- & -- & (a) & $(a)$ & -- & - & $\cdots$ & (a) \\
\hline 2 & - & -- & - & - & - & -- & - & -- & $(a)$ & - & -- & (a) & (a) & - & - & - & $(a)$ \\
\hline 3 & $-\infty$ & - & -. & -- & 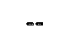 & -- & -- & -- & (a) & -- & -- & (a) & $(a)$ & - & -- & -- & (a) \\
\hline 4 & -- & -- & - & $(c)$ & -- & -- & -- & - & (a) & -- & -- & (a) & $(a)$ & - & - & - & (a) \\
\hline 5 & -- & -- & -- & - & $=$ & $-\cdot$ & -- & - & - & -- & -- & - & (a) & -- & $\cdots$ & $(a)$ & (a) \\
\hline 6 & -- & - & -- & -- & - & - & - & - & -- & -- & -- & - & -- & -- & -- & (a) & (a) \\
\hline 7 & -- & -- & - & -- & - & 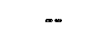 & -- & $\infty$ & -- & - & - & - & -- & -- & - & $(a)$ & $\cdots$ \\
\hline 8 & - & - & - & -- & -- & -- & -- & -- & -- & -- & -- & -- & -- & $\cdots$ & - & (a) & -- \\
\hline 9 & -- & -- & - & -- & -- & - & -- & - & -- & - & -- & -- & -- & -- & $-\infty$ & (a) & $\cdots$ \\
\hline 10 & - & - & -- & - & -- & -- & - & -- & -- & - & -- & -- & -- & $\cdots$ & $-\infty$ & $(a)$ & -- \\
\hline 11 & 0.02 & 0.02 & 0.17 & \multicolumn{2}{|c|}{ (c) 0.15} & -- & 0.12 & 0.21 & 0.12 & 0.21 & 0.13 & 0.51 & 0.10 & 0.16 & 0.15 & (b) & 0.06 \\
\hline 12 & -- & -- & .11 & 0.01 & .15 & 0.23 & -- & - & .02 & .02 & .01 & .06 & .01 & -- & .01 & (b) & -. \\
\hline 13 & - & - & -- & -- & -- & -- & - & - & -- & - & -- & (b) & -- & .26 & -- & (b) & -- \\
\hline 14 & - & -- & - & -- & - & -- & -- & - & -- & - & -- & (b) & -- & .21 & - & (b) & -- \\
\hline 15 & - & - & - & -- & -- & -- & -- & -- & -- & -- & -- & (b) & - & -- & -- & (b) & - \\
\hline 16 & -- & - & -- & - & - & $\ldots$ & - & - & - & -- & -- & (b) & -- & - & $=$ & (b) & -- \\
\hline 17 & -- & - & -- & -- & $-\infty$ & -. & -- & - & - & -- & -- & (b) & -- & -. & -- & (b) & -- \\
\hline 18 & .07 & .08 & .04 & .07 & .10 & .10 & .16 & .11 & .21 & .31 & .15 & (b) & .13 & .15 & .20 & (b) & .08 \\
\hline 19 & - & - & - & (c) & -. & -- & -- & - & .02 & -- & -- & (b) & -. & .01 & -. & .45 & -- \\
\hline 20 & -- & - & -- & - & $\cdots$ & -- & - & - & - & - & -- & (b) & -- & .14 & -- & (a) & -- \\
\hline 21 & .01 & - & - & .07 & -- & .11 & .03 & .22 & .10 & .14 & .14 & (b) & .11 & .05 & .14 & .02 & .01 \\
\hline 22 & $\infty$ & $-\infty$ & -- & -- & - & -- & -. & .02 & .01 & - & -. & $f_{.63}$ & -- & - & -. & -. & -- \\
\hline 23 & - & $-\infty$ & - & -- & -- & - & -- & - & -- & -- & -- & (a) & -- & -- & -- & -- & -- \\
\hline 24 & -- & $\cdots$ & - & - & - & -. & -- & - & -- & - & $\cdots$ & (a) & - & -- & $\ldots$ & - & $\infty$ \\
\hline 25 & - & $-\infty$ & - & - & - & -- & -- & - & - & - & -- & (a) & - & 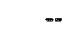 & -- & -- & -- \\
\hline 26 & -- & $-\infty$ & -- & -- & - & -- & -- & - & -- & -- & - & (a) & - & -- & -- & -- & - \\
\hline 27 & - & $\cdots$ & $=$ & - & - & $=$ & -. & -- & -- & -. & -. & -- & -- & -- & $=$ & - & $\ldots$ \\
\hline 28 & -. & - & - & -- & -. & -- & -. & -- & - & - & -- & -- & $=$ & - & -. & -- & - \\
\hline 29 & -- & - & -- & (c) & -- & - & -- & - & -- & -- & -- & -. & - & -. & -- & -- & -- \\
\hline 30 & -- & - & - & - & - & $=$ & - & -- & .29 & .20 & .05 & .33 & .12 & .02 & .15 & .05 & .13 \\
\hline Total & 0.10 & 0.10 & 0.32 & 0.15 & 0.40 & 0.44 & 0.31 & 0.56 & 0.77 & 0.88 & 0.48 & 1.53 & 0.47 & 1.00 & 0.65 & 0.52 & 0.28 \\
\hline
\end{tabular}


TABLE 7.--Precipitation data from Weather Senvice Nuclear Support Office network stations--Continued.

\begin{tabular}{|c|c|c|c|c|c|c|c|c|c|c|c|c|c|c|c|c|c|}
\hline \multirow[b]{2}{*}{ DAY } & \multicolumn{17}{|c|}{ Station } \\
\hline & MER & RV & $4 \mathrm{JA}$ & DRA & $\mathrm{CS}$ & W5B & MV & UCC & $40 \mathrm{MN}$ & $\mathrm{TS} 2$ & BJY & $\mathrm{A} 12$ & PHS & PM1 & $\mathrm{LF}_{2}$ & $\mathrm{LF} 1$ & PU13 \\
\hline \multicolumn{18}{|c|}{ MAY 1983} \\
\hline 1 & 0.08 & 0.06 & 0.04 & 0.04 & 0.07 & 0.11 & 0.11 & 0.28 & 0.25 & 0.30 & 0.36 & 0.45 & 0.18 & (b) & 0.19 & 0.12 & 0.09 \\
\hline 2 & -- & .01 & -- & (c) & - & -- & -- & -. & .02 & -- & - & .01 & .01 & 0.32 & .01 & .01 & .09 \\
\hline 3 & -- & -- & -- & - & -- & - & .- & -- & -- & -- & -- & -. & - & (a) & -- & -- & - \\
\hline 4 & -. & - & -- & $\ldots$ & -- & -- & - & -- & - & - & - & -. & -. & (a) & -- & -- & -- \\
\hline 5 & -- & -- & -- & - & - & - & .- & -- & -- & -- & - & .02 & - & -- & .01 & - & - \\
\hline 6 & - & -- & - & -- & - & -- & -- & .. & -- & $\ldots$ & -- & -- & - & -- & .01 & -- & -. \\
\hline 7 & -. & -- & - & -- & - & -- & -- & -- & -- & - & -- & -. & -- & - & - & -- & -- \\
\hline 8 & -- & -- & -- & - & - & -- & - & -- & -- & -- & -- & -. & -- & -- & -- & -. & -- \\
\hline 9 & -. & -- & $\ldots$ & .. & - & - & - & -- & -- & -- & -- & .. & -. & $\ldots$ & -- & -- & - \\
\hline 10 & -- & -. & -- & (c) & - & - & -. & -- & - & - & -- & -- & -- & -- & -- & -- & -- \\
\hline 11 & -- & -. & -- & - & - & -- & -- & -. & -- & - & -- & .. & -- & -. & -- & -- & -- \\
\hline 12 & -- & -- & -- & -. & -.. & -- & -- & -- & -- & -- & - & -. & -- & -- & -- & -. & -. \\
\hline 13 & -- & -- & -- & -. & - & $\infty$ & -- & -. & -. & - & $\infty$ & -. & -. & -- & -- & -- & - \\
\hline 14 & $\cdots$ & -- & -- & -- & - & $=$ & -- & - & $\ldots$ & - & -- & -- & $\ldots$ & -- & -- & -- & - \\
\hline 15 & -- & -- & -- & -- & - & - & $\ldots$ & -- & -. & -- & -- & -- & -- & -- & .- & - & - \\
\hline 16 & -. & $\infty$ & -- & - & - & - & $\infty$ & -. & .- & ... & - & .. & .- & -- & -- & -. & -- \\
\hline 17 & -- & - & -- & -- & - & -. & $\ldots$ & -- & -- & - & -- & -- & -. & .. & -- & -- & -- \\
\hline 18 & --. & - & -- & - & -- & .. & $\ldots$ & .- & _. & -. & .. & -. & -. & .. & - & -- & .. \\
\hline 19 & -. & - & -. & - & $\infty$ & -- & $\ldots$ & $\ldots$ & .- & -- & -- & $=$ & $\infty$ & .. & -- & -- & $=$ \\
\hline 20 & -- & -- & -- & - & - & - & -- & -- & -- & -- & - & -- & - & -- & -- & -- & -- \\
\hline 21 & -. & -.. & -. & - & -- & - & -. & $=-$ & -- & -. & -- & -- & -- & -- & $\ldots$ & -. & -- \\
\hline 22 & - & -. & - & -- & -. & -- & -. & .- & -- & -- & -- & -- & - & -- & - & -- & -- \\
\hline 23 & - & -- & $-\infty$ & -. & -- & - & $\infty$ & -- & -- & -- & $-\infty$ & -- & -. & -- & -. & -- & -- \\
\hline 24 & -- & -- & -- & - & - & -- & $\ldots$ & -. & -- & -- & -- & -- & -- & -. & $\ldots$ & -. & (a) \\
\hline 25 & - & -- & -- & - & - & - & -- & $\ldots$ & -- & -- & -- & -- &.- & -- & -- & -- & (a) \\
\hline 26 & $=$ & -- & -- & $\ldots$ & $-\infty$ & - & $\ldots$ & .. & -- & - & -. & -. & -. & -- & - & . & (a) \\
\hline 27 & - & -- & -- & - & - & - & -- & -- & -- & -- & -- & -- & - & -- & -- & -- & (a) \\
\hline 28 & -- & -- & -. & -. & - & - & - & -. & -- & -. & .- & -- & -- & -- & - & -- & (a) \\
\hline 29 & -- & -- & -- & -- & -- & - & -. & -- & .. & $\ldots$ & -- & -- & $\ldots$ & .- & -- & -- & (a) \\
\hline 30 & -- & -. & $\ldots$ & -- & $\ldots$ & - & -- & $\ldots$ & -. & -- & -- & -- & -- & - & $\ldots$ & .- & (a) \\
\hline 31 & -. & - & -- & -- & -- & - & - & -. & -- & $=$ & $-\cdots$ & - & - & -- & -- & .. & (a) \\
\hline Total & 0.08 & 0.07 & 0.04 & 0.04 & 0.07 & 0.11 & 0.11 & 0.28 & 0.27 & 0.30 & 0.36 & 0.48 & 0.19 & 0.32 & 0.22 & 0.14 & 0.18 \\
\hline
\end{tabular}


TABLE 7.--Precipitation data from Weather Service Nuclear Support Office network stations--Continued.

\begin{tabular}{|c|c|c|c|c|c|c|c|c|c|c|c|c|c|c|c|c|c|}
\hline \multirow[b]{2}{*}{ DAY } & \multicolumn{17}{|c|}{ Station } \\
\hline & MER & RV & $4 \mathrm{JA}$ & DRA & $\mathrm{CS}$ & W5B & MV & UCC & $40 \mathrm{MN}$ & $\mathrm{TS} 2$ & BJY & A12 & PHS & PM1 & $\mathrm{LF} 2$ & $\mathrm{LF} 1$ & PU13 \\
\hline \multicolumn{18}{|c|}{ JUNE 1983} \\
\hline 1 & - & -- & -- & -- & - & - & -- & -- & -- & -- & - & -- & -- & (b) & -- & - & -- \\
\hline 2 & $\ldots$ & -- & - & - & -- & - & -- & -- & -- & -. & $\infty$ & - & 0.03 & (b) & -- & - &.- \\
\hline 3 & -- & -- & - & -. & -- & - & -- & -- & -- & -- & -- & - & -- & (b) & -- & -- & -- \\
\hline 4 & .. & -- & -- & -- & -. & -. & -- & -. & -- & -- & -. & -- & .02 & (b) & -. & -. & -- \\
\hline 5 & -- & -- & -- & -- & -- & -- & -- & -- & $-\cdots$ & $\ldots$ & - & - & - & (b) & - & -- & -- \\
\hline 6 & -. & - & -. & -- & -. & -. &.- & -. & $\ldots$ & .. & -- & -- & -. & (b) & -- & - & -- \\
\hline 7 & -- & -- & - & - & 0.03 & 0.02 & -.. & 0.03 & -- & 0.08 & -. & - & -- & $f_{0.12}$ & -- & - & - \\
\hline 8 & -. & -- & $\ldots$ & -- & -. & -- & -- & -. & -- & -- & -- & - & -- & (b) & -- & -- & -- \\
\hline 9 & - & - & -- & -- & -. & -- & -- & -- & - & -. & -- & - & -- & (b) & -. & - & $=$ \\
\hline 10 & -- & - & -- & - & -- & -- & -- & -. & $-\infty$ & -- & -- & -- & -- & (b) & -- & -- & -- \\
\hline 11 & -- & - & - & $\ldots$ & -. & -- & -- & -- & -- & -. & -- & - & -- & (b) & - & -- & 0.01 \\
\hline 12 & -. & -. & - & -. & -- & -. & .- & -. & - & -. & -- & - & -. & (b) & -- & -. & -. \\
\hline 13 & -- & -. & -.. & -. & -. & -- & -- & -- & -. & -. & -- & - & $-\infty$ & (b) & -- & -- & -. \\
\hline 14 & -- & -- & - & .. & -- & -- & $-\infty$ & -- & -- & -- & $\ldots$ & -- & -- & (b) & - & - & - \\
\hline 15 & -- & -- & -- & -. & -- & -. & -. & -. & - & -- & -. & $=$ & -. & (b) & - & - & - \\
\hline 16 & -- & -- & -- & -. & -- & -- & -- & -- & -. & -- & $\ldots$ & -- & -- & (b) & - & -- & -. \\
\hline 17 & -. & -- & -. & -- & -- & -- & -. & -. & -. & -. & -- & -. & -. & (b) & -- & -- & -. \\
\hline 18 & -- & -- & -- & - & .. & - & -- & -- & -- & $\ldots$ & -. & $\ldots$ & -- & (b) & - & - & -. \\
\hline 19 & -- & -- & -- & -- & -- & -- & -. & -- & -- & -- & -. & -- & -- & (b) & -- & - & $\ldots$ \\
\hline 20 & - & -. & $\infty$ & -- & - & $-\infty$ & -. & $=$ & - & .. & -- & -- & - & (b) & $\ldots$ & -. & - \\
\hline 21 & - & $\infty$ & -- & -- & -- & -- & -. & -- & -. & $=$ & -- & - & $-\infty$ & (b) & - & - & -- \\
\hline 22 & - & $\ldots$ & -. & -- & -- & $\infty$ & $=$ & $-\infty$ & - & -. & - & -- & $\infty$ & (b) & - & 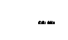 & - \\
\hline 23 & -- & - & - & -- & -. & - & -- & -- & -- & -- & -- & $-\infty$ & $=$ & (b) & - & $-\infty$ & -- \\
\hline 24 & -- & -- & -- & -- & -- & -- & -- & - & -- & -- & - & -- & -. & (b) & -- & -- & -. \\
\hline 25 & -- & $=$ & -. & -- & -- & $=$ & - & - & - & - & -- & - & -. & (b) & - & $-\infty$ & -- \\
\hline 26 & -- & -- & -- & -- & -- & -- & -- & -. & -- & -- & -- & -- & -- & (b) & - & -. & .. \\
\hline 27 & - & -- & - & -- & $\infty$ & -. & -. & -. & -. & -- & -- & - & $\ldots$ & (b) & - & -- & -- \\
\hline 28 & -. & -- & -- & -- & -- & .. & -- & -- & - & -- & - & $-\infty$ & -- & (b) & - & - & -- \\
\hline 29 & -. & -- & -. & -. & -- & -- & -- & -. & -. & -.. & -- & -- & $\ldots$ & (b) & - & -- & -- \\
\hline 30 & -- & -- & - & - & $\ldots$ & -. & - & $\ldots$ & -. & $\ldots$ & -. & -- & -. & ${ }^{d .05}$ & -- & - & -- \\
\hline Total & 0 & 0 & 0 & 0 & 0.03 & 0.02 & 0 & 0.03 & 0 & 0.08 & 0 & 0 & 0.05 & $e_{0.17}$ & 0 & 0 & 0.01 \\
\hline
\end{tabular}


TABLE 7.--Precipitation data from Weather Service Nuclear Support Office network stations--Continued.

Station

\begin{tabular}{|c|c|c|c|c|c|c|c|c|c|c|c|c|c|c|c|c|c|}
\hline DAY & MER & RV & 4JA & DRA & $\mathrm{CS}$ & W5B & $\mathrm{MV}$ & UCC & $40 \mathrm{MN}$ & $\mathrm{TS} 2$ & B.JY & $\mathrm{A} 12$ & PHS & PM1 & LF2 & LF1 & PU13 \\
\hline \multicolumn{18}{|c|}{ JULY 1983} \\
\hline 1 & -. & $\ldots$ & $-\infty$ & - & $=$ & $-\infty$ & $\ldots$ & -- & $=$ & $\infty$ & $-\infty$ & - & -- & (b) & $-\infty$ & $-\infty$ & -- \\
\hline 2 & -- & -- & -- & - & - & -- & - & -- & -- & -- & -- & -- & -- & (b) & -- & -- & -- \\
\hline 3 & -- & $-\infty$ & - & -- & -- & - & -- & - & - & -- & -- & $-\infty$ & -- & (b) & -- & -- & -- \\
\hline 4 & -- & -- & -- & -- & -- & -- & -- & -- & -- & $\ldots$ & -- & $-\infty$ & -- & (b) & - & -- & - \\
\hline 5 & -- & -- & - & - & - & - & - & - & -- & -- & -- & -- & -- & (b) & - & -- & -- \\
\hline 6 & $\ldots$ & -- & -- & -- & - & -- & -- & -- & -- &.- & - & -- & -- & (b) & $=$ & -- & -- \\
\hline 7 & -- & -- & -- & -- & - & - & - & -- & -- & $\ldots$ & -- & -- & -- & $d_{0.05}$ & -- & -- & -- \\
\hline 8 & -- & -- & - & - & -- & -- & - & - & -- & - & - & -- & -- & $(a)$ & -- & -- & -- \\
\hline 9 & -- & -. & -- & -- & -- & - & -- & $-\infty$ & -- & -. & -- & -- & -- & (a) & -- & -- & -- \\
\hline 10 & -- & -- & -- & -- & - & -- & -- & - & -- & -- & -- & -- & -- & $(a)$ & -- & -- & -- \\
\hline 11 & -- & .. & -- & - & -- & $-\infty$ & - & -- & -- & -- & -- & -. & -- & (a) & -. & -- & -- \\
\hline 12 & -- & -- & -- & -- & -- & -- & - & -- & -- & -- & -- & -- & -- & (a) & -- & -- & -- \\
\hline 13 & -- & -. & -- & $\ldots$ & $-\infty$ & -- & - & -- & -- & - & -. & -- & -- & (a) & -- & -- & -. \\
\hline 14 & -. & $=$ & $-\infty$ & - & -- & -- & - & -- & - & -- & -. & -- & -- & (a) & -. & -- & -- \\
\hline 15 & -- & -. & -- & -- & -- & -- & -- & -- & -- & -- & -- & -- & -- & (a) & -- & -- & -- \\
\hline 16 & -. & -. & .- & -- & -- & $=$ & - & $\infty$ & $=$ & $\infty$ & a- & - & -. & (a) & $\ldots$ & - & . \\
\hline 17 & -. & -- & -- & -.- & -- & -- & -- & -- & -. & .. & -_- & - & -- & (a) & -. & -- & -- \\
\hline 18 & $\ldots$ & $\ldots$ & $\ldots$ & - & $=$ & -- & $\ldots$ & -- & -- & -- & -- & $\alpha$ & -- & (a) & -- & $\ldots$ & .- \\
\hline 19 & -- & -. & - & $-\infty$ & .. & $\ldots$ & $\ldots$ & - & - & -- & -. & $-\infty$ & -- & (a) & - & $\ldots$ & -- \\
\hline 20 & -- & -- & -- & -- & -- & -- & -. & -- & -- & -- & -- & -- & .- & (a) & -- & $=$ & -. \\
\hline 21 & $\ldots$ & -- & - & (c) & - & - & -- & -- & -- & -- & .- & -. & -- & (a) & -- & .- & $\ldots$ \\
\hline 22 & -- & -- & -. & - & -. & -. & -. & -- & - & - & -- & - & -- & (a) & -- & -- & -- \\
\hline 23 & $=$ & - & $-\infty$ & $\ldots$ & - & -. & - & - & - & -- & - & .- & .- & (a) & $=$ & $=$ & -. \\
\hline 24 & -- & -. & -. & -- & -- & -- & -- & -- & -- & -- & -. & -- & -. & (a) & -- & -- & -. \\
\hline 25 & -- & -. & -- & -- & -- & -- & .- & -- & -- & -- & -- & -- & -. & (a) & - & - & -- \\
\hline 26 & -- & -. & -. & -. & -. & -. & $-\infty$ & $\ldots$ & - & $\ldots$ & - & -- & -. & (a) & -. & -. & -. \\
\hline 27 & - & - & -- & -- & -- & -. & $\ldots$ & -. & - & -- & - & -- & .. & (a) & - & -- & -- \\
\hline 28 & - & $=-$ & $=$ & - & -- & $=$ & -. & - & $=$ & $\cdots$ & - & -. & .. & (a) & - & - & -. \\
\hline 29 & - & -- & -- & -- & -- & -- & -- & -- & -- & -- & -- & -- & -. & (a) & - & -- & -- \\
\hline 30 & - & - & -. & (c) & - & - & -- & -- & -- & $-\infty$ & -. & -- & -. & (a) & -- & - & -- \\
\hline 31 & -- & - & $-\infty$ & (c) & -- & 0.03 & -- & -- & $\ldots$ & - & - & - & 0.09 & ${ }^{d} .05$ & -- & - & 0.02 \\
\hline Total & 0 & 0 & 0 & (c) & 0 & 0.03 & 0 & 0 & 0 & 0 & 0 & 0 & 0.09 & $e^{e_{0}} .10$ & 0 & 0 & 0.02 \\
\hline
\end{tabular}


TABLE 7.--Precipiration data from Weather Service Nuclear Support Office network stations--Continued.

\begin{tabular}{|c|c|c|c|c|c|c|c|c|c|c|c|c|c|c|c|c|c|}
\hline \multirow[b]{2}{*}{$\mathrm{DAY}$} & \multicolumn{17}{|c|}{ Station } \\
\hline & MER & $\mathbf{R V}$ & $4 \mathrm{JA}$ & DRA & $\mathrm{CS}$ & W5B & $\mathrm{MV}$ & $\mathrm{UCC}$ & $40 \mathrm{MN}$ & $\mathrm{TS} 2$ & BJY & A12 & PHS & PM1 & $\mathrm{LF} 2$ & LFI & PU13 \\
\hline \multicolumn{18}{|c|}{ AUGUST 1983} \\
\hline 1 & $\infty$ & 0.01 & $-\infty$ & (c) & -- & -- & -- & - & - & - & - & -- & - & (b) & - & 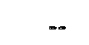 & 0.01 \\
\hline 2 & - & - & $-\infty$ & - & $-\infty$ & -- & -- & -- & .. & -- & - & $-\infty$ & - & (b) & -- & -- & - \\
\hline 3 & -. & $\ldots$ & -- & -. & .. & -- & $\ldots$ & - & .- & -. & - & -- & (b) & (b) & .. & -- & -. \\
\hline 4 & -- & -- & -- & ... & -- & -- & -- & -- & -- & -- & .. & -- & (b) & (b) & .- & .- & -- \\
\hline 5 & -- & -- & - & -- & -- & -- & -- & - & $\ldots$ & - & -- & (b) & (b) & (b) & -- & -- & -- \\
\hline 6 & 0.26 & .- & 0.11 & 0.06 & 0.56 & 0.32 & 0.60 & 0.26 & (b) & (b) & 0.60 & (b) & (b) & (b) & (b) & 0.13 & .23 \\
\hline 7 & .01 & -- & .09 & .02 & .01 & -. & - & -- & (b) & (b) & -. & (b) & (b) & (b) & (b) & -- & (b) \\
\hline 8 & - & -- & .01 & $\ldots$ &.- & - & -. & -- & 60.35 & $f_{0.31}$ & -- & (b) & (b) & (b) & $f_{0.46}$ & -- & (b) \\
\hline 9 & .24 & .02 & .04 & .10 & .05 & .09 & .10 & .03 & .05 & .02 & .35 & $f_{1.20}$ & $f_{0.52}$ & (b) & .04 & .11 & (b) \\
\hline 10 & .36 & .52 & .01 & .52 & .10 & .73 & .07 & .12 & .20 & .08 & .15 & .30 & .11 & $d_{1.30}$ & .10 & .13 & $f_{.28}$ \\
\hline 11 & -- & -- & -- & -- & -- & -- & - & - & .03 & -- & -. & .01 & - & (b) & -. & -. & (b) \\
\hline 12 & -. & -. & -- & -- & .. & .- & -. & -. & $\ldots$ & - & - & -- & - & (b) & .. & $\ldots$ & (b) \\
\hline 13 & -- & -- & .. & -. & -. & -. & -- & -. & .. & - & -- & -- & $\infty$ & (b) & -. & $-\infty$ & (b) \\
\hline 14 & -- & .. & -- & .04 & .02 & .05 & .08 & .05 & .01 & .05 & .15 & .17 & .01 & (b) & .01 & -. & (b) \\
\hline 15 & .01 & .04 & .08 & (c) & -. & $=$ & .06 & - & .03 & .01 & .02 & .06 & .01 & (b) & .35 & .13 & (b) \\
\hline 16 & .13 & .17 & .18 & .19 & 1.18 & .10 & .73 & .47 & .28 & .35 & 1.01 & 1.19 & .40 & (b) & .14 & .18 & (b) \\
\hline 17 & .40 & .38 & .12 & .43 & .42 & .35 & .24 & .23 & .08 & .19 & .35 & .22 & .16 & (b) & .48 & (b) & (b) \\
\hline 18 & 3.63 & 2.98 & 3.22 & 3.52 & 3.47 & 1.86 & 2.72 & 2.15 & 2.99 & .86 & 2.30 & 2.75 & 1.86 & (b) & 2.04 & (b) & (b) \\
\hline 19 & -. & .31 & .03 & .45 & -. & .24 & .05 & .18 & .24 & - & -. & (b) & .28 & (b) & .. & (b) & (b) \\
\hline 20 & $=$ & - & - & .04 & $=$ & - & $\ldots$ & .01 & .08 & $\ldots$ & - & (b) & - & (b) & -- & (b) & (b) \\
\hline 21 & -- & - & - & -- & .- & .. & .- & .01 & ... & - & -- & $f_{1.10}$ & -. & 1.99 & .- & $f_{3.75}$ & $f_{1.58}$ \\
\hline 22 & - & $\ldots$ & -. & -- & -- & .. & .- & - & .. & -. & - & -. & - & (a) & $\ldots$ & - & -. \\
\hline 23 & - & $\infty$ & -- & -. & -. & -- & -- & $=$ & -- & -- & -- & -- & -- & (a) & -- & - & -. \\
\hline 24 & -. & - & -- & - & -. & -- & -- & -- & - & -- & - & - & -- & (a) & -- & -- & - \\
\hline 25 & -. & $\infty$ & -- & -- & .. & $=$ & -- & -. & $=$ & -- & -. & $\ldots$ & - & (a) & -- & -- & -. \\
\hline 26 & -- & -. & .. & .- & -- & .. & $\ldots$ & .. & -- & $\ldots$ & -- & -. & $\ldots$ & (a) & -- & .. & .. \\
\hline 27 & -. & -. & - & -- & .. & .. & .. & -. & $\ldots$ & m. & - & $\ldots$ & m. & (a) & $\infty$ & $=$ & -. \\
\hline 28 & -- & $\ldots$ & - & $\ldots$ & $=$ & .- & .. & .. & $\ldots$ & -- & - & $=$ & $\ldots$ & (a) & $=$ & -- & .- \\
\hline 29 & -- & -. & - & -- & -. & -- & -. & -- & -- & -- & - & -- & -- & (a) & -- & -. & -. \\
\hline 30 & -- & $\ldots$ & m & - & -- & .. & $\ldots$ & -- & $\ldots$ & $-\infty$ & $=$ & - & $\ldots$ & (a) & -- & - & - \\
\hline 31 & -. & - & -- & -- & -- & -- & -- & -- & -. & - & -- & - & - & (a) & -- & - & -- \\
\hline Total & 5.04 & 4.42 & 3.89 & 5.37 & 5.81 & 3.74 & 4.65 & 3.51 & 4.34 & 1.87 & 4.93 & 7.00 & 3.35 & 3.29 & 3.62 & 4.43 & 2.10 \\
\hline
\end{tabular}


TABLE 7.--Precipitation data from Weather Senice Nuclear Support Office network stations--Continued.

\begin{tabular}{|c|c|c|c|c|c|c|c|c|c|c|c|c|c|c|c|c|c|}
\hline \multirow[b]{2}{*}{ DAY } & \multicolumn{17}{|c|}{ Station } \\
\hline & MER & RV & $4 J A$ & DRA & $\mathrm{CS}$ & W5B & MV & UCC & $40 \mathrm{MN}$ & $\mathrm{TS} 2$ & BJY & $\mathrm{A} 12$ & PHS & PM1 & $\mathrm{LF} 2$ & LF1 & PU13 \\
\hline \multicolumn{18}{|c|}{ SEPTEMBER 1983} \\
\hline 1 & -- & -- & -- & -- & -- & -- & - & -- & $-\infty$ & -- & -- & -- & -- & (a) & -- & $-\infty$ & (a) \\
\hline 2 & -. & -- & -- & -- & -- & -- & -- & -- & -- & $\cdots$ & -- & -. & -. & (a) & - & -- & (a) \\
\hline 3 & -- & -- & -- & -- & -- & - & -- & -- & -- & -- & -- & -- & -- & (a) & - & -- & (a) \\
\hline 4 & -- & - & -- & -- & - & - & -- & - & -- & -- & -- & -- & -- & (a) & - & $-\infty$ & (a) \\
\hline 5 & $\cdots$ & -- & -- & -- & -- & - & -- & -- & -- & -- & -- & -- & - & (a) & -- & -- & (a) \\
\hline 6 & -- & - & -- & - & - & - & -- & -- & -- & .. & -- & -- & -. & (a) & -- & $\ldots$ & (a) \\
\hline 7 & -- & $\ldots$ & $-\infty$ & -- & -- & -- & -. & -- & - & -. & $\infty$ & -. & -- & (a) & -- & $\ldots$ & (a) \\
\hline 8 & -. & -- & -- & -- & -- & -- & -- & -. & -- & .- & -- & -- & -- & (a) & - & - & (a) \\
\hline 9 & -- & -- & -- & -- & $-\infty$ & $\ldots$ & $\cdots$ & -. & -- & -- & -- & -. & -. & (a) & -- & -- & (a) \\
\hline 10 & -- & -- & - & -- & -- & -- & -- & -- & -- & -- & -- & -- & -- & (a) & -- & - & (a) \\
\hline 11 & -. & -- & -- & -- & - & - & -- & - & -.. & -- & -. & -- & -- & (a) & -- & -. & (a) \\
\hline 12 & -- & -- & -- & -- & -- & - & - & -- & -- & -- & -- & -- & -. & (a) & -- & -- & (a) \\
\hline 13 & -. & -- & -- & - & -- & -- & -- & - & $-\infty$ & -- & -. & -- & - & (a) & $=$ & - & (a) \\
\hline 14 & -- & -- & - & -- & -- & -- & -- & - & - & $-\cdots$ & $-\infty$ & -. & -- & (a) & - & - & (a) \\
\hline 15 & -- & - & -- & - & -- & - & -- & -- & -- & - & -- & - & - & (a) & - & -- & (a) \\
\hline 16 & -- & -- & -- & - & -- & -- & - & -- & -- & -- & -- & -- & -- & (a) & -- & -- & (a) \\
\hline 17 & -. & -. & -- & -- & -- & - & -- & -- & - & $-\infty$ & -- & -. & -- & (a) & - & -- & (a) \\
\hline 18 & -. & -. & -- & - & -- & -- & - & $=$ & $\infty$ & $\ldots$ & -- & -. & -- & (a) & $\ldots$ & -- & (a) \\
\hline 19 & -- & -- & -- & -- & -- & -- & - & - & -- & -- & -. & -- & -- & (a) & -- & -- & (a) \\
\hline 20 & -- & - & -- & -- & -- & -- & -- & -- & - & - & - & -. & -- & $(a)$ & - & - & (a) \\
\hline 21 & -- & -- & -- & - & -- & -- & - & -- & -- & -. & $\ldots$ & -- & $\ldots$ & $(a)$ & - & -- & (a) \\
\hline 22 & -. & -. & -. & -- & -- & -- & -- & - & -- & -- & $\ldots$ & -. & -. & (a) & - & -- & (a) \\
\hline 23 & - & -- & -. & -. & $\ldots$ & - & -- & -- & - & -- & -. & -. & - & (a) & -. & -- & (a) \\
\hline 24 & -- & - & -- & -- & -- & -- & -- & -- & -- & -- & -- & -- & -- & (a) & -- & -- & (a) \\
\hline 25 & -- & - & 0.06 & -- & -- & -- & 0.22 & - & 0.09 & 0.17 & 0.01 & 0.11 & -- & (b) & 0.09 & 0.10 & (b) \\
\hline 26 & 0.60 & 0.34 & .17 & 0.59 & 0.45 & 0.35 & .71 & 0.42 & 1.03 & .62 & .56 & .60 & 0.43 & (b) & .76 & (b) & (b) \\
\hline 27 & -. & -. & - & - & -. & -- & - & -- & -- & -- & - & -. & .02 & $(b)$ & - & (b) & (b) \\
\hline 28 & -- & - & $-\infty$ & (c) & .01 & -- & - & - & -. & -- & -- & .02 & -- & (b) & .04 & (b) & (b) \\
\hline 29 & .49 & -- & -- & .35 & - & .81 & -- & .53 & .01 & .04 & .32 & .33 & .10 & (b) & .02 & (b) & (b) \\
\hline 30 & .05 & .01 & .04 & .07 & .03 & .06 & .10 & .04 & .18 & .16 & .10 & .36 & .07 & ${ }^{d} 0.63$ & .22 & $d_{1.06}$ & $d_{1.18}$ \\
\hline Total & 1.14 & 0.35 & 0.27 & 1.01 & 0.49 & 1.22 & 1.03 & 0.99 & 1.31 & 0.99 & 0.99 & 1.42 & 0.62 & $e_{0.63}$ & 1.13 & ${ }^{e} 1.16$ & $e^{e_{1.18}}$ \\
\hline
\end{tabular}


TABLE 7.--Precipitation data from Weather Service Nuclear Support Office nework stations--Continued.

\begin{tabular}{|c|c|c|c|c|c|c|c|c|c|c|c|c|c|c|c|c|c|}
\hline \multirow[b]{2}{*}{ DAY } & \multicolumn{17}{|c|}{ Station } \\
\hline & MER & RV & 4JA & DRA & $\mathrm{CS}$ & W5B & MV & $\mathrm{UCC}$ & $40 \mathrm{MN}$ & $\mathrm{TS} 2$ & BJY & $\mathrm{A} 12$ & PHS & PM1 & LF2 & LF1 & PU13 \\
\hline \multicolumn{18}{|c|}{ OCTOBER 1983} \\
\hline 1 & 0.14 & 0.07 & 0.08 & 0.19 & 0.06 & 0.15 & (b) & 0.09 & 0.85 & 0.11 & 0.06 & 0.52 & 0.16 & (b) & 0.48 & (b) & (b) \\
\hline 2 & -. & - & - & - & - & - & $f_{0.06}$ & - & .03 & .01 & - & .01 & - & $d_{0.50}$ & .02 & $d_{0.47}$ & $d_{0.25}$ \\
\hline 3 & -- & -- & -- & - & - & - & (a) & -- & - & -- & -- & -- & -- & (a) & -- & -- & (a) \\
\hline 4 & -- & -- & $\ldots$ & -- & -- & -- & (a) & 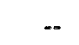 & -- & $\ldots$ & - & -- & -- & (a) & -- & -- & (a) \\
\hline 5 & -- & -- & -- & (c) & - & -- & - & -- & -- & -- & -- & -- & -- & (a) & -- & -- & (a) \\
\hline 6 & -- & -- & - & -- & - & - & -- & $\ldots$ & $-\infty$ & $\ldots$ & - & -- & $=$ & $\ldots$ & $=$ & -- & -- \\
\hline 7 & -- & -. & -- & -- & -- & -- & - & - & -. & -- & .. & -- & $\ldots$ & - & - & -- & -- \\
\hline 8 & $\ldots$ & -- & - & $\ldots$ & -- & -. & -- & -- & -- & -. & -- & -- & -- & -. & - & -- & -- \\
\hline 9 & 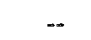 & -- & -- & -- & -- & -- & - & -- & - & -- & -- & -- & -- & -. & - & -- & -- \\
\hline 10 & -- & -. & - & -- & - & -- & - & -- & $\ldots$ & -- & .. & - & -. & -- & -. & $\ldots$ & -- \\
\hline 11 & -. & -- & - & -. & -- & $\ldots$ & -- & - & $-\infty$ & -. & -. & -- & -- & $\ldots$ & $-\infty$ & $\ldots$ & -- \\
\hline 12 & -- & -- & -- & -- & -- & -- & -- & -- & $\ldots$ & - & -- & -- & -- & -- & - & -. & $\ldots$ \\
\hline 13 & -. & -- & -- & -- & -- & -- & -- & -- & $\ldots$ & -- & -- & -- & - & -- & - & -- & -- \\
\hline 14 & -- & - & -- & -- & -- & -- & -- & -- & $\cdots$ & -. & -- & - & -. & -. & $\ldots$ & -- & - \\
\hline 15 & -- & -. & - & -- & -- & -- & - & -- & - & -. & - & -- & -. & -- & -- & -- & -- \\
\hline 16 & - & -- & - & -- & -- & -- & - & - & 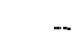 & -. & .. & -- & - & -- & .. & -- & -. \\
\hline 17 & -- & - & - & -- & -- & -- & -- & -- & -. & $\ldots$ & -- & -- & -- & -. & -- & .- & -. \\
\hline 18 & -- & -- & - & -- & -- & -- & - & -- & - & -- & -- & -- & - & -- & $\ldots$ & -- & -- \\
\hline 19 & -- & -- & -- & -- & -- & - & -- & -- & $\ldots$ & -- & $=$ & -- & $-\infty$ & -- & $=$ & -- & - \\
\hline 20 & -- & $-\infty$ & -- & $-\infty$ & -- & - & -- & -- & -. & -- & $-\infty$ & -- & - & -- & -- & -- & -- \\
\hline 21 & -- & -- & -- & -. & - & - & - & -- & $\ldots$ & -. & -. & $\ldots$ & -. & -- & - & -- & - \\
\hline 22 & -. & $\ldots$ & -- & -- & -- & -- & - & 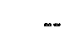 & $\ldots$ & $\ldots$ & -. & -- & - & $-\infty$ & $\ldots$ & .. & -- \\
\hline 23 & -- & -- & - & -- & -- & -- & -- & -. & $\ldots$ & $\ldots$ & -- & -- & - & -- & -. & -- & -. \\
\hline 24 & -. & $\ldots$ & -- & -- & -- & -- & -- & - & - & -- & .. & -- & -. & $\ldots$ & -. & $\ldots$ & -- \\
\hline 25 & -- & -- & -- & - & -- & -- & - & -- & - & -- & -. & -- & -- & -- & -- & -- & - \\
\hline 26 & -. & -- & - & -- & -- & -- & - & -. & $\cdots$ & -. & $\ldots$ & -- & -- & -- & -- & -- & -- \\
\hline 27 & -. & $\infty$ & -- & -- & -. & -- & -- & -- & $\infty$ & - & -. & -- & -- & - & $\infty$ & -- & -. \\
\hline 28 & -- & .- & -- & -- & -- & .. & -. & -- & -- & -- & .. & -- & - & .. & .. & -. & - \\
\hline 29 & .- & - & .. & -- & .. & -- & -. & -. & -- & -. & .. & -. & -. & -. & -. & .. & -- \\
\hline 30 & -- & -- & -- & -. & -- & - & -- & -- & $-\infty$ & -. & $\ldots$ & -- & - & - & -. & -. & $\ldots$ \\
\hline 31 & -- & -. & -- & -. & -- & - & -- & -- & $\ldots$ & -- & .. & -- & -- & -- & -- & -- & -- \\
\hline Total & 0.14 & 0.07 & 0.08 & 0.19 & 0.06 & 0.15 & 0.06 & 0.09 & 0.88 & 0.12 & 0.06 & 0.53 & 0.16 & $e^{e} .50$ & 0.50 & $e_{0.47}$ & $e_{0.25}$ \\
\hline
\end{tabular}


TABLE 7.--Precipitation data from Weather Service Nuclear Support Office network stationsm-Continued.

\begin{tabular}{|c|c|c|c|c|c|c|c|c|c|c|c|c|c|c|c|c|c|}
\hline \multirow[b]{2}{*}{ DAY } & \multicolumn{17}{|c|}{ Station } \\
\hline & MER & RV & \$IA & DRA & $\mathrm{CS}$ & W5B & MV & $\mathrm{UCC}$ & $40 \mathrm{MN}$ & TS2 & BJY & $\mathrm{A} 12$ & PHS & PMI & LF2 & LF1 & PU13 \\
\hline \multicolumn{18}{|c|}{ NOVEMBER 1983} \\
\hline 1 & 0.05 & $\cdots$ & $-\infty$ & 0.32 & -- & - & -- & - & - & - & $-\infty$ & -- & $-\infty$ & - & 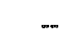 & -- & - \\
\hline 2 & .02 & -- & $\cdots$ & .01 & - & - & - & -. & -- & -- & - & $\cdots$ & -- & -- & -- & - & - \\
\hline 3 & -- & -- & -. & - & -- & -. & -. & $=$ & $\cdots$ & -- & - & -. & $=$ & -- & - & -- & -- \\
\hline 4 & -- & - & -- & - & - & -- & - & -- & $\cdots$ & - & - & -- & -- & - & -- & - & -- \\
\hline 5 & - & -- & $-\infty$ & $=$ & $-\infty$ & $-\infty$ & $\infty$ & - & - & - & - & $-\infty$ & $=$ & - & $\infty$ & - & -- \\
\hline 6 & - & -- & -- & - & - & - & - & -- & $\cdots$ & -- & - & -- & $\cdots$ & -- & - & -- & $-\infty$ \\
\hline 7 & .05 & $-\infty$ & $=$ & .05 & -- & 0.01 & - & - & -- & - & - & - & -- & $=$ & -- & -. & -- \\
\hline 8 & -- & $\cdots$ & -. & -- & -- & -- & -- & -- & - & -- & - & - & - & (a) & -- & (a) & $\cdots$ \\
\hline 9 & - & - & -- & -- & -- & -- & -- & $\cdots$ & -- & - & - & - & -- & (a) & -- & (a) & -- \\
\hline 10 & - & $\cdots$ & $-\infty$ & -. & $\cdots$ & $\cdots$ & $\cdots$ & $=$ & -- & -- & - & -- & - & (a) & - & (a) & - \\
\hline 11 & - & - & $\cdots$ & -- & 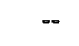 & - & $\cdots$ & - & $\cdots$ & -- & - & -- & $\ldots$ & (a) & - & (a) & -- \\
\hline 12 & -- & - & - & -- & -- & -- & $\cdots$ & -- & -- & -- & - & - & - & (a) & - & (a) & -- \\
\hline 13 & $=$ & $a$ & - & $=$ & $\ldots$ & $=$ & - & oe & - & $=$ & $\cdots$ & $\infty$ & -- & (a) & - & (a) & -. \\
\hline 14 & -- & -- & -- & - & -- & - & -- & -. & -- & -- & - & -- & $\cdots$ & (a) & -- & (a) & -- \\
\hline 15 & - & $-\infty$ & $\infty$ & $\infty$ & -- & $=$ & $-\cdots$ & $\infty$ & $m$ & -- & - & $\infty$ & - & $(a)$ & -- & (a) & -- \\
\hline 16 & -- & - & $-\infty$ & $=$ & - & 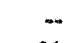 & -- & $\cdots$ & -- & - & -- & - & - & $(a)$ & -- & (a) & - \\
\hline 17 & .05 & 0.02 & -- & .06 & 0.02 & .01 & 0.02 & 0.06 & 0.02 & 0.09 & 0.07 & 0.01 & 0.01 & (b) & 0.02 & (b) & 0.08 \\
\hline 18 & $\infty$ & $\infty$ & $=$ & -- & -. & - & -. & -- & .01 & - & -- & - & - & (b) & $=$ & (b) & .01 \\
\hline 19 & $-\infty$ & -- & - & -- & -- & $-\cdots$ & -- & -- & -- & - & -- & + & -- & (b) & -- & (b) & -- \\
\hline 20 & .10 & .01 & .01 & .01 & .03 & .03 & .02 & .01 & .04 & .05 & - & .27 & .01 & (b) & .03 & (b) & -. \\
\hline 21 & .02 & -. & $-\infty$ & .03 & - & $\cdots$ & .01 & - & .03 & .02 & $=$ & - & .07 & (b) & .02 & $f_{.23}$ & .05 \\
\hline 22 & -- & - & -- & -- & + & -- & - & - & -- & $-\infty$ & -- & - & - & (b) & -. & - & -- \\
\hline 23 & - & - & -. & $=$ & -. & $=$ & -. & - & -- & $=$ & -- & -- & -- & (b) & - & - & -- \\
\hline 24 & .13 & .43 & .37 & .11 & .46 & .19 & .83 & .44 & .44 & .52 & .46 & .57 & .33 & (b) & .33 & .27 & .14 \\
\hline 25 & .37 & .23 & .17 & .25 & .19 & .30 & .16 & .22 & .13 & .17 & .18 & .05 & .08 & (b) & .06 & .01 & .18 \\
\hline 26 & - & - & -- & -- & -- & $\cdots$ & -- & -- & -- & - & -- & -- & -- & (b) & - & - & -. \\
\hline 27 & -- & $\infty$ & -- & $-\infty$ & $=$ & - & -. & -. & -- & .- & $=$ & - & $-\infty$ & ${ }^{d .59}$ & -- & $\ldots$ & .07 \\
\hline 28 & $-\infty$ & - & $\cdots$ & -- & -- & -- & -- & -- & $-\infty$ & -- & - & -- & - & (a) & $\infty$ & -- & -. \\
\hline 29 & -- & - & -- & -- & -- & -. & - & - & 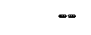 & -. & $-\infty$ & - & - & (a) & -- & - & -- \\
\hline 30 & - & - & -- & $\cdots$ & -- & $\infty$ & -- & - & - & - & $\ldots$ & -- & -. & $(a)$ & $\cdots$ & $\cdots$ & $=$ \\
\hline Total & 0.79 & 0.69 & 0.55 & 0.84 & 0.70 & 0.54 & 1.04 & 0.73 & 0.67 & 0.85 & 0.71 & 0.90 & 0.50 & e.59 & 0.46 & 0.51 & 0.53 \\
\hline
\end{tabular}


TABLE 7.--Precipitation data from Weather Service Nuclear Support Office network stationsw-Continued.

Station

\begin{tabular}{|c|c|c|c|c|c|c|c|c|c|c|c|c|c|c|c|c|c|}
\hline \multirow[b]{2}{*}{ DAY } & \multicolumn{17}{|c|}{ Station } \\
\hline & MER & $\mathbb{R V}$ & $4 J \mathbf{A}$ & DRA & CS & W5B & MV & UCC & $40 \mathrm{MN}$ & TS2 & BJY & A12 & PHS & PM1 & $\mathrm{LF}_{2}$ & $\mathbf{L F I}$ & PU13 \\
\hline \multicolumn{18}{|c|}{ DECEMBER 1983} \\
\hline 1 & -- & (a) & -- & - & - & - & -- & - & - & - & - & - & -- & (a) & - & -- & - \\
\hline 2 & - & (b) & - & -- & -. & -- & - & -. & 0.01 & - & 0.01 & 0.01 & - & (b) & 0.01 & -- & -- \\
\hline 3 & 0.04 & 80.02 & 0.03 & 0.02 & 0.07 & 0.05 & 0.36 & 0.02 & .33 & 0.29 & .09 & .35 & 0.09 & $d_{0.20}$ & .25 & 0.15 & 0.15 \\
\hline 4 & -- & (a) & -- & - & - & -- & -. & - & - & -- & .. & - & - & (a) & - & -. & -- \\
\hline 5 & -- & (a) & - & -- & -. & -- & -- & -- & - & $\ldots$ & -- & -- & -- & (a) & -- & -. & -. \\
\hline 6 & -- & (a) & - & $-\infty$ & -. & -- & - & $-\infty$ & $-\infty$ & $-\infty$ & -- & -- & - & (a) & - & -. & -. \\
\hline 7 & -- & $\ldots$ & - & -- & .. & $-\infty$ & .- & $-\infty$ & -- & -- & - & -. & -- & (a) & -- & -. & -. \\
\hline 8 & -. & - & $-\sim$ & -. & -- & -- & -.. & -- & - & -. & -- & -- & - & -. & -- & -- & -- \\
\hline 9 & $=$ & - & $\infty$ & (c) & - & -. & -. & -- & - & $=$ & -. & $-\infty$ & - & $-\infty$ & -- & - & -. \\
\hline 10 & -- & - & - & - & -. & -- & -. & .. & -- & - & -- & .02 & -- & - & .- & -. & -. \\
\hline $\mathbb{1 1}$ & -- & $-\infty$ & -- & -- & $\ldots$ & -- & -- & -- & - & - & $\ldots$ & -- & $-\infty$ & -. & - & -. & -- \\
\hline 12 & -- & 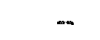 & $-\infty$ & -. & -.. & $=$ & -- & -- & - & -- & $\ldots$ & -- & -- & $-\infty$ & $-\infty$ & -- & -- \\
\hline 13 & -- & - & -- & -. & -- & -- & -- & -. & -- & -- & -- & -- & -- & -. & -- & -- & -. \\
\hline 14 & -- & - & -. & $\ldots$ & -. & -. & .- & -- & - & -- & -. & -. & -- & - & -. & -. & -- \\
\hline 15 & -- & - & -- & -- & -- & -- & -. & -. & -- & - & -- & -- & - & - & -- & -- & -- \\
\hline 16 & -- & $-\infty$ & - & -- & $\infty$ & $-\infty$ & -. & -- & $-\infty$ & $=$ & -- & $=$ & -- & $\rightarrow$ & $=$ & -- & -. \\
\hline 17 & -- & $-\infty$ & $=$ & -- & $\ldots$ & - & -. & -- & - & -. & $-\infty$ & - & -- & -. & - & $\ldots$ & -- \\
\hline 18 & -- & - & -- & -. & -- & -- & .. & -. & -- & - & -- & -. & $-\infty$ & -. & $\ldots$ & - & - \\
\hline 19 & $\infty$ & $-\infty$ & -. & -- & -. & .. & - & -- & -. & - & -. & $\infty$ & -- & - & -. & -- & -. \\
\hline 20 & -- & -. & -- & -. & -- & -- & -- & -. & - & $\cdots$ & -- & -. & - & -. & -- & -- & -- \\
\hline 21 & -- & $-\infty$ & -- & -- & -- & -- & -. & -- & - & $\ldots$ & -- & -- & -- & $\infty$ & -. & $-\infty$ & -- \\
\hline 22 & $\ldots$ & $-\infty$ & -- & -- & - & -- & - & -- & -. & - & -- & -- & -- & - & -- & -- & - \\
\hline 23 & -- & - & - & -- & -- & -- & -- & -- & -- & - & - & - & -- & -. & -- & - & - \\
\hline 24 & .19 & .21 & .28 & .24 & .27 & .19 & .43 & .30 & .36 & .38 & .30 & .32 & .43 & (b) & .25 & (b) & .36 \\
\hline 25 & .47 & .47 & .53 & .56 & .52 & .32 & 1.02 & .70 & .80 & .65 & .74 & .79 & .61 & (b) & .76 & (b) & .46 \\
\hline 26 & -. & $-\infty$ & -- & - & -- & $-\infty$ & .05 & $=$ & .15 & .05 & .04 & .08 & .04 & (b) & .12 & (b) & .05 \\
\hline 27 & -- & - & .01 & -- & - & - & -- & -- & - & - & - & -- & .02 & $f .81$ & -. & $f_{1.10}$ & -. \\
\hline 28 & -- & - & -- & -- & - & - & -- & -- & -. & $-\infty$ & -- & - & -- & - & - & (a) & - \\
\hline 29 & - & - & -- & -- & - & -- & -- & -- & $=$ & - & -- & -- & - & - & -- & (a) & -- \\
\hline 30 & - & -- & -- & - & -- & - & - & -- & -- & - & - & $=$ & -- & -- & - & (a) & - \\
\hline 31 & $\infty$ & - & -. & - & $=$ & - & $=$ & $=$ & - & - & - & - & -- & - & $=$ & (a) & - \\
\hline Total & 0.70 & $e_{0.70}$ & 0.85 & 0.82 & 0.86 & 0.56 & 1.86 & 1.02 & 1.65 & 1.37 & 1.18 & 1.57 & 1.19 & $e^{e} 1.01$ & 1.39 & 1.25 & 1.02 \\
\hline
\end{tabular}


TABLE 7.--Precipitation data from Weather Senire Nuclear Suppont Office network stations-Continued.

\begin{tabular}{|c|c|c|c|c|c|c|c|c|c|c|c|c|c|c|c|c|c|}
\hline \multirow[b]{2}{*}{ DAY } & \multicolumn{17}{|c|}{ Station } \\
\hline & MER & RV & $4 \mathrm{~J} \mathrm{~A}$ & DRA & $\mathrm{CS}$ & W5B & MV & $\mathrm{UCC}$ & $40 \mathrm{MN}$ & TS2 & $\mathrm{BJY}$ & $\mathrm{A} 12$ & PHS & $\mathrm{PMI}$ & LF2 & LF1 & PU13 \\
\hline \multicolumn{18}{|c|}{ JANUARY 1984} \\
\hline 1 & - & -- & - & -- & -- & -- & -- & -- & -- & -- & - & -- & -- & -- & -. & (a) & -- \\
\hline 2 & $=$ & -- & $-\infty$ & -- & -- & - & - & -- & $=$ & -- & $\infty$ & - & - & - & -. & (a) &.- \\
\hline 3 & -- & -- & - & -- & -- & -- & -- & -- & -- & -- & - & -- & -- & -- & -. & (a) & -- \\
\hline 4 & -. & $\ldots$ & - & -- & .. & - & - & -- &.- & -- & - & -- & - & -- & -- & (a) & -- \\
\hline 5 & -- & -- & -- & -- & - & - & - & -- & -- & - & -- & - & - & -- & -- & -- & -- \\
\hline 6 &.- & -- & -- & -- & -- & - & -- & -- & -- & -- & -- & -- & - & -- & -. & -- & -- \\
\hline 7 & .. & -- & -- & -- & -- & -- & -- & $-\infty$ & $\ldots$ & -- & -. & -- & - & -- & -- & - & -. \\
\hline 8 & -- & -- & -- & -- & -- & -. & -- & -- & -- & -- & -- & -- & - & -- & -- & -. & -- \\
\hline 9 & .. & - & -. & - & -. & $\ldots$ & -- & -. & - & $\ldots$ & - & .. & - & -- & -. & -- & $\ldots$ \\
\hline 10 & -- & - & - & -- & -- & -- & -- & $\ldots$ & -- & -- & -- & -- & - & -. & $\ldots$ & -- & -- \\
\hline 11 & -- & -. & -. & - & -- & -- & -- & -- & -- & - & - & -- & - & -- & -- & -- & -- \\
\hline 12 & -- & -- & -- & -- & -- & -- & -. & - & -- & -- & -- & 0.03 & -- & -. & -- & -- & -- \\
\hline 13 & -- & -- & -. & 0.02 & - & -- & - & -- & $=$ & -- & -. & -- & -- & -- & - & $\infty$ & -- \\
\hline 14 & $\ldots$ & a. & -. & - & - & a. & - & -- & .- & -. & -. & - & 0.04 & -. & $\ldots$ & -- & -- \\
\hline 15 & -. & -- & -- & -. & -. & -- & - & - & -. & -. & -. & -- & -- & 0.01 & -- & -. & -- \\
\hline 16 & $=$ & -- & -- & -. & -- & -- & - & - & - & - & -- & - & -- & -- & - & -- & -- \\
\hline 17 & -. & -. & -- & -- & - & - & - & -- & .. & -- & .. & -- & -- & -- & -- & -- & -. \\
\hline 18 & -- & -- & -- & -- & - & -- & -- & -- & -- & -- & - & -- & -- & -. & -- & -- & -- \\
\hline 19 & -- & -- & -- & - & -- & -- & -- & -- & -. & -. & -- & -- & - & -- & -. & -. & -. \\
\hline 20 & -- & -- & -- & -- & -- & -- & - & - & -- & -- & - & -- & -- & -- & -. & - & -- \\
\hline 21 & -- & -. & -- & .- & $\ldots$ & $\ldots$ & -. & -- & -- & -- & -. & .02 & - & -. & -. & .. & -- \\
\hline 22 & -- & -- & -- & -- & -- & -- & -. & -. & -- & -- & -- & -- & -- & .04 & -. & .. & -- \\
\hline 23 & -. & -. & -- & -. & -- & -- & -- & -- & -. & -. & -- & -- & -- & -- & -- & -- & -- \\
\hline 24 & -- & -. & $-\infty$ & -. & - & - & -- & -- & -- & -- & -. & - & $-\infty$ & -- & -. & -. & -- \\
\hline 25 & -- & -- & -- & -- & - & -- & - & - & -- & -- & - & - & -- & - & - & -- & -- \\
\hline 26 & -- & -. & -- & -- & -- & -- & -. & $=$ & -- & - &.- & -- & - & -- & -. & -- & -. \\
\hline 27 & -- & -. &.- & -. & .. & -. & -. & -. & -. & .. & .. & -- & -- & -- & -. & .. & .. \\
\hline 28 & -. & -. & -- & -- & -. & -- & -. & -- & -- & $\ldots$ & -. & -- & -. & -- & .. & -. & -- \\
\hline 29 & -. & -- & -. & -. & 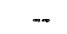 & -. & -- & -. &.- & -- & -. & -. & -- & -- & -- & $m$ & -. \\
\hline 30 & -- & -. & -. & -. & -. & -. & -- & -- & -- & -. & -. & -- & - & -- & -- & -- & -- \\
\hline 31 & -- & -. & $=$ & $\cdots$ & $\cdots$ & 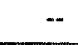 & - & $\infty$ & -- & - & - & -- & - & -- & - & -- & -- \\
\hline Total & 0 & 0 & 0 & 0.02 & 0 & 0 & 0 & 0 & 0 & 0 & 0 & 0.05 & 0.04 & 0.05 & 0 & 0 & 0 \\
\hline
\end{tabular}


TABLE 7.--Precipitation data from Weather Service Nuclear Support Office nenwork stations--Continued.

\begin{tabular}{|c|c|c|c|c|c|c|c|c|c|c|c|c|c|c|c|c|c|}
\hline \multirow[b]{2}{*}{ DAY } & \multicolumn{17}{|c|}{ Station } \\
\hline & MER & RV & $4 J A$ & DRA & $\mathrm{CS}$ & W5B & MV & UCC & $40 \mathrm{MN}$ & TS2 & BJY & A12 & PHS & PM1 & $\mathbb{L F}_{2}$ & LFI & PU13 \\
\hline \multicolumn{18}{|c|}{ FEBRUARY 1984} \\
\hline 1 & -- & - & -- & -- & -- & - & - & -- & -- & - & -- & - & - & -- & -- & - & -- \\
\hline 2 & -- & - & - & - & - & - & - & -- & -- & -- & -- & -- & -- & - & $\ldots$ & -- & -- \\
\hline 3 & $-\infty$ & -- & -- & -- & -- & -- & -- & - & -- & - & -- & -- & -- & - & -- & -- & -- \\
\hline 4 & - & -- &.- & $\ldots$ & -- & -- & - & - & -- & -- & -. & -- & - & -- & -- & -- & - \\
\hline 5 & $\cdots$ & -- & -- & -- & -- & -- & - & -- & -- & -- & -- & -- & -- & -- & -- & -- & -- \\
\hline 6 & $-\infty$ & -- & -- & -- & -- & - & -- & -- & -- & -. & -. & .. & -. & $\ldots$ & -. & -. & -- \\
\hline 7 & -- & -- & -- & -- & -- & - & - & - & - & -- & -- & -- & -- & (a) & -- & -- & -- \\
\hline 8 & $\ldots$ & -. & -- & -. & -- & -- & -- & -- & -. & -. & -.. & .- & -- & (a) & -. & -. & .. \\
\hline 9 & -- & -- & -. & -- & -. & - & - & -- & -- & -- & -- & -- & - & (a) & -. & -- & -- \\
\hline 10 & 0.07 & 0.05 & 0.08 & 0.06 & 0.17 & 0.09 & 0.08 & 0.07 & 0.05 & 0.07 & 0.08 & 0.23 & 0.07 & (b) & 0.07 & (b) & 0.10 \\
\hline 11 & -- & -- & - & -- & -- & -- & -- & -- & -. & -- & -. & .. & -- & (b) & -. & (b) & -- \\
\hline 12 & -- & $-\infty$ & -- & -- & -- & -- & $\infty$ & - & -- & - & -- & -- & -- & (b) & -- & (b) & - \\
\hline 13 & .. & -- & $\ldots$ & .. & -. & .. & -- & -. & -. & -. & .. & $\ldots$ & -- & (b) & -. & (b) & -- \\
\hline 14 & .02 & .01 & .07 & .01 & .05 & .05 & .05 & .02 & .05 & .02 & .02 & .07 & .06 & (b) & .07 & (b) & .01 \\
\hline 15 & -- & -- & -- & -- & -. & - & - & -- & -- & - & -. & -. & -- & (b) & -. & (b) & - \\
\hline 16 & .03 & .01 & - & .07 & .01 & .02 & .01 & -- & -- & -. & -. & .16 & .03 & $f_{.30}$ & .01 & $f_{.10}$ & -- \\
\hline 17 & -- & -- & -- & -- & -- & - & -- & -- & -- & $\ldots$ & -. & -. & -- & $(a)$ & -- & $-\infty$ & -- \\
\hline 18 & -. & -- & -- & -- & $-\infty$ & $-\infty$ & - & $-\infty$ & - & - & -. & -- & -- & $(a)$ & -. & - & -- \\
\hline 19 & -- & -- & -. & -- & .- & -- & - & - & -- & -- & -- & -- & - & (a) & -- & -. & -- \\
\hline 20 & -- & -- & -- & -- & -- & -- & - & - & - & - & -. & -. & - & $(a)$ & -- & -- & -- \\
\hline 21 & -- & -. & -- & -- & -. & $=$ & - & -- & $-\infty$ & -- & -- & -- & - & (a) & -. & -- & -- \\
\hline 22 & -- & -- & -- & -. & -- & -- & -- & -- & -- & - & -- & -- & -. & (a) & -- & -- & -- \\
\hline 23 & $=$ & $\ldots$ & -- & -- & -. & -- & - & -- & - & - & $-\infty$ & .. & -- & (a) & -- & -- & -- \\
\hline 24 & -- & -- & .. & -. & -. & -- & - & -- & - & - & -- & -. & -- & (a) & -. & -. & $\ldots$ \\
\hline 25 & - & $\ldots$ & -. & -. & -. & -- & - & - & -- & -- & -- & -- & $=$ & (a) & -- & -- & -- \\
\hline 26 & -- & -- & -. & -- & -- & -- & -. & -- & -- & -- & -- & -. & $\ldots$ & (a) & -. & -- & $\ldots$ \\
\hline 27 & -. & -- & -. & -- & -- & -- & -- & -- & -- & -- & -- & -- & . & (a) & $=$ & $\ldots$ & -- \\
\hline 28 & -- & -- & -- & -- & $\ldots$ & -. & - & -- & -- & $-\infty$ & - & -- & $-\infty$ & (a) & - & -- & -- \\
\hline 29 & -. & -. & -- & -- & -- & -- & -- & -- & -- & -- & -- & -- & -- & (a) & - & -- & -- \\
\hline Total & 0.12 & 0.07 & 0.15 & 0.14 & 0.23 & 0.16 & 0.14 & 0.09 & 0.10 & 0.09 & 0.10 & 0.46 & 0.16 & 0.30 & 0.15 & 0.10 & 0.11 \\
\hline
\end{tabular}


TABLE 7.--Precipitation data from Weather Service Nuclear Support Office network stations--Continued.

\begin{tabular}{|c|c|c|c|c|c|c|c|c|c|c|c|c|c|c|c|c|c|}
\hline \multirow[b]{2}{*}{ DAY } & \multicolumn{17}{|c|}{ Station } \\
\hline & MER & RV & $4 J \mathrm{~A}$ & DRA & $\mathrm{CS}$ & W5B & MV & UCC & $40 \mathrm{MN}$ & $\mathrm{TS} 2$ & BJY & $\mathrm{A} 12$ & PHS & PM1 & $\mathrm{LF} 2$ & LF1 & PU13 \\
\hline \multicolumn{18}{|c|}{ MARCH 1984} \\
\hline $\mathbb{1}$ & - & $-\infty$ & - & - & $\infty$ & $=$ & -- & -- & -- & -. & -- & $-\infty$ & - & (a) & -. & .- & -. \\
\hline 2 & -- & - & -- & -- & -- & -- & -- & - & - & -- & - & -- & -- & (a) & -- & -- & -- \\
\hline 3 & - & - & - & $-\infty$ & -- & - & - & -- & -- & -. & -- & -- & -- & (a) & -- & - & -- \\
\hline 4 & - & - & - & - & $\cdots$ & -- & - & - & - & - & - & -- & -- & (a) & -- & -- & - \\
\hline 5 & -- & - & -- & - & - & $\ldots$ & $\sim$ & - & -- & -- & - & -- & -- & (a) & -- & -- & -- \\
\hline 6 & $=$ & -- & $\cdots$ & -- & - & -- & $\ldots$ & -. & $-\infty$ & -- & -- & - & - & $(a)$ & -- & -- & - \\
\hline 7 & -- & -- & -- & -- & -- & - & -- & -- & -- & -- & -- & -- & -- & -- & -- & -- & -- \\
\hline 8 & - & - & - & $=$ & $-\infty$ & $\infty$ & - & - & -- & - & $=$ & -. & - & -. & -. & -- & ... \\
\hline 9 & -. & -- & - & $\ldots$ & -. & $\ldots$ & $\ldots$ & - & $-\infty$ & - & - & $\ldots$ & - & -. & -- & - & -- \\
\hline 10 & - & - & - & - & - & $\cdots$ & -- & -- & - & -- & - & -- & -- & -- & -- & -- & -- \\
\hline 11 & -- & -- & -- & $=$ & $=$ & $\ldots$ & - & - & - & - & -- & - & - & -- & -- & $=$ & -. \\
\hline 12 & -- & -- & - &.- & $\ldots$ & $\ldots$ & - & -- & -. & -- & $\cdots$ & -. & -- & -- & -- & -- & -. \\
\hline 13 & -. & - & - & (c) & -- & -- & -. & $\ldots$ & -. & -- & -- & - & -. & -. & -. & -- & 0.01 \\
\hline 14 & .. & -- & - & (c) & -. & -. & $\ldots$ & -. & $\ldots$ & -. & - & 0.03 & - & 0.02 & -. & -- & .04 \\
\hline 15 & -- & -- & -- & - & $\cdots$ & -- & - & - & -. & - & -- & - & $\cdots$ & -- & - & - & -- \\
\hline 16 & - & $\ldots$ & - & -- & -. & -- & - & -- & -- & -- & -- & -. & -- & -- & .. & -- & .- \\
\hline 17 & -- & $-\infty$ & -- & $-\infty$ & -- & -. & -- & -- & -. & -- & -. & -- & -- & -- & - & -. & -- \\
\hline 18 & -. & -- & -- & $\alpha=$ & -. & - & $=$ & $=$ & $=$ & $\infty$ & - & -- & $\infty$ & - & -- & - & - \\
\hline 19 & - & -- & - & -. & -. & -- & -. & - & -- & -- & -- & -- & - & -. & - & -- & -. \\
\hline 20 & $=$ & $=$ & $\cdots$ & - & - & $\ldots$ & - & - & - & - & -- & - & $\ldots$ & - & - & $-\infty$ & - \\
\hline 21 & $=\infty$ & $\ldots$ & - & $\ldots$ & -. & -- & -- & -- & -- & -- & -- & -- & -- & -- & -- & $\infty$ & -. \\
\hline 22 & -- & -- & -- & -- & -- & -- & -- & - & -. & $\ldots$ & -. & - & .. & $\ldots$ & - & - & -. \\
\hline 23 & -. & -- & -- & .. & -. & -- & -- & -- & -- & -- & $=$ & - & - & $=$ & -- & - & -. \\
\hline 24 & -- & -- & -- & $\ldots$ & - & $\ldots$ & - & -. & -- & -. & -- & -- & -- & -- & - & -- & -. \\
\hline 25 & -. & -- & -. & -. & -- & - & - & -- & - & - & -- & - & .- & -. & -- & -- & -. \\
\hline 26 & - & -- & -- & $\ldots$ & - & -. & - & -. & $\ldots$ & $\ldots$ & -- & -. & .. & -. & - & -- & -- \\
\hline 27 & $\ldots$ & -- & $\ldots$ & -- & - & -- & - & - & $\ldots$ & - & -- & - & -. & $\ldots$ & - & -- & -- \\
\hline 28 & - & -- & -. & - & -- & $\ldots$ & -- & -- & a. & $\ldots$ & $\ldots$ & - & - & - & - & -. & $\ldots$ \\
\hline 29 & 0.05 & -. & -. & 0.09 & 0.01 & $-\infty$ & 0.01 & 0.01 & -. & -- & $\ldots$ & .05 & -- & - & -- & $\ldots$ & -- \\
\hline 30 & - & - & -. & $=$ & - & $=$ & -- & -- & -- & - & $=$ & -. & -. & -- & -- & $-\infty$ & - \\
\hline 31 & - & - & - & -- & - & - & - & -- & - & 0.01 & -. & .04 & -- & - & - & -- & -- \\
\hline Total & 0.05 & 0 & 0 & 0.09 & 0.01 & 0 & 0.01 & 0.01 & 0 & 0.01 & 0 & 0.12 & 0 & 0.02 & 0 & 0 & 0.05 \\
\hline
\end{tabular}


TABLE 7.--Precipitation data from Weather Service Nuclear Support Office network stations--Continued.

\begin{tabular}{|c|c|c|c|c|c|c|c|c|c|c|c|c|c|c|c|c|c|}
\hline \multirow[b]{2}{*}{ DAY } & \multicolumn{17}{|c|}{ Station } \\
\hline & MER & RV & $4 \mathrm{JA}$ & DRA & $\mathrm{CS}$ & W5B & $\mathrm{MV}$ & $\mathrm{UCC}$ & $40 \mathrm{MN}$ & TS2 & BJY & $\mathrm{A} 12$ & PHS & PMI & $\mathrm{LF} 2$ & LF1 & PU13 \\
\hline \multicolumn{18}{|c|}{ APRLL 1984} \\
\hline 1 & 0.01 & - & - & (c) & 0.01 & 0.02 & 0.02 & 0.04 & 0.09 & 0.06 & -- & 0.20 & 0.01 & 0.22 & 0.09 & 0.02 & 0.05 \\
\hline 2 & -- & - & - & - & -- & -- & -- & -- & -- & -- & -- & -- & - & -- & -- & - & -. \\
\hline 3 & -- & - & -- & $\ldots$ & - & - & -- & - & -- & - & -. & -- & - & -- & $\ldots$ & - & -- \\
\hline 4 & -- & $\ldots$ & $\infty$ & - & - & -- & - & -- & -- & -- & - & $-\infty$ & $-\infty$ & - & $-\infty$ & $m$ & -- \\
\hline 5 & - & -- & -- & - & - & - & - & -- & -- & -- & -- & -- & -- & -- & - & - & -- \\
\hline 6 & .30 & 0.01 & 0.01 & 0.04 & .30 & .03 & .16 & .01 & .04 & .18 & 0.02 & .16 & .11 & .04 & .06 & .01 & .03 \\
\hline 7 & - & - & - & - & - & -. & -. & -- & - & - & -- & -- & - & -- & -- & -- & - \\
\hline 8 & -- & -. & $\ldots$ & -- & -- & -- & -- & -- & $\ldots$ & - & $-\infty$ & -- & - & $\ldots$ & $\ldots$ & $\ldots$ & -. \\
\hline 9 & - & $=$ & -- & $\ldots$ & -. & $\ldots$ & -. & -. & -- & $-\infty$ & $\ldots$ & -- & $\infty$ & $=$ & -- & -- & - \\
\hline 10 & -- & -- & -- & - & -. & -. & -- & -. & -- & -- & -- & -- & -- & -- & -- & -- & - \\
\hline 11 & .- & -. & -- & - & $\ldots$ & .. & -. & $\ldots$ & - & - & - & - & -- & -- & - & -- & -- \\
\hline 12 & -- & - & -. & - & - & . & $\ldots$ & -. & -- & -- & -- & -. & -. & - & -- & - & $-\infty$ \\
\hline 13 & -- & - & -- & $\ldots$ & - & $\ldots$ & $\ldots$ & .. & -- & -- & - & -- & -- & -- & - & - & $\ldots$ \\
\hline 14 & $\ldots$ & -- & - & -- & -- & -. & -- & -- & $\ldots$ & $\infty$ & -. & -- & - & -- & - & -- & -- \\
\hline 15 & - & -- & - & -- & - & -. & - & -- & -- & - & - & -- & -- & - & $\cdots$ & -. & -- \\
\hline 16 & -- & - & - & - & $-\infty$ & -. & -. & -. & $\ldots$ & $-\infty$ & - & $=$ & $-\infty$ & - & $\ldots$ & $=$ & $\ldots$ \\
\hline 17 & -- & $=$ & - & -- & $=$ & -- & -. & -- & $\ldots$ & -- & - & -- & -- & $\ldots$ & - & -- & -- \\
\hline 18 & -. & -. & $-\infty$ & -- & -- & .. & -. & -- & -. & -. & - & -- & -- & - & .. & $=$ & $m$ \\
\hline 19 & $\ldots$ & -- & -- & -- & - & -. & .01 & -. & .01 & $=$ & - & .05 & .02 & .02 & .02 & -- & -- \\
\hline 20 & -- & - & - & -- & -- & -- & -- & -- & .. & $\ldots$ & - & -- & -- & - & $\ldots$ & -. & - \\
\hline 21 & -- & - & $-\infty$ & -. & -- & -- & - & -- & - & - & - & $=$ & -- & -. & $\ldots$ & -. & $\ldots$ \\
\hline 22 & - & - & -- & -- & - & -- & -- & $\infty$ & $=$ & -- & - & .. & -- & - & -- & -- & - \\
\hline 23 & - & -- & -- & -. & -- & -- & -- & -- & - & - & -- & -. & -- & - & $\infty$ & a- & or \\
\hline 24 & -- & $=$ & $=$ & -. & - & - & -. & $\ldots$ & $=$ & -- & -- & $\ldots$ & - & - & -- & -- & - \\
\hline 25 & -- & -- & -- & -. & $\ldots$ & - & -. & -. & -- & -- & - & -. & - & - & -- & -- & - \\
\hline 26 & -. & $\infty$ & $=\infty$ & -- & - & $\ldots$ & $\ldots$ & - & $\ldots$ & -. & $=$ & -- & - & .. & -. & .- & - \\
\hline 27 & -- & 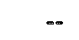 & -- & - & $\ldots$ & $\ldots$ & - & -- & -. & $=$ & $=$ & -- & - & -. & - & - & -- \\
\hline 28 & $-\infty$ & -- & -. & -- & - & - & - & - & -- & -- & -- & $-\infty$ & $\infty$ & $-\infty$ & - & 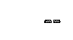 & $m$ \\
\hline 29 & $\ldots$ & -. & -- & a- & $=$ & -- & -- & $=$ & -- & $\ldots$ & 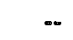 & $=$ & $-\infty$ & - & -- & -- & -- \\
\hline 30 & -- & -- & -- & -. & $\ldots$ & $\ldots$ & $\cdots$ & -- & -- & -- & -- & -- & -- & $\ldots$ & - & $\cdots$ & -- \\
\hline Total & 0.31 & 0.01 & 0.01 & 0.04 & 0.31 & 0.05 & 0.19 & 0.05 & 0.14 & 0.24 & 0.02 & 0.41 & 0.14 & 0.28 & 0.17 & 0.03 & 0.08 \\
\hline
\end{tabular}


TABLE 7.--Precipitation data from Weather Sevice Nuclear Support Office network stations--Continued.

\begin{tabular}{|c|c|c|c|c|c|c|c|c|c|c|c|c|c|c|c|c|c|}
\hline \multirow[b]{2}{*}{ DAY } & \multicolumn{17}{|c|}{ Station } \\
\hline & MER & RV & $4 \mathrm{JA}$ & DRA & CS & W5B & MV & UCC & $40 \mathrm{MN}$ & $\mathrm{TS} 2$ & BJY & $\mathrm{A} 12$ & PHS & PM1 & $L F 2$ & LF1 & PU13 \\
\hline \multicolumn{18}{|c|}{ MAY 1984} \\
\hline 1 & .- & .- & .- & -. & -- & -- & -- & -. & -- & -- & -- & - & -- & -- & -- & -- & .. \\
\hline 2 & -- & -- & -- & -- & -- & -- & -- & -- & -- & .. & .- & -. & .- & .- & -- & -- & -- \\
\hline 3 & -- & -. & -. & -- & -- & -- & -- & -. & -- & -. & -- & -- & -- & -. & -- & .. & .- \\
\hline 4 & .- & .. & -- & -- & - & -- & -- & -- & -- & -- & -- & -- & -- & -- & -- & -- & -- \\
\hline 5 & -- & -- & -- & -- & -- & -- & -- & -- & -- & -- & -- & -- & -- & -- & -- & -- & -- \\
\hline 6 & -- & -- & -. & -- & -. & -- & -- & -- & -- & -- & -- & -- & -- & -- & -- & -- & -- \\
\hline 7 & .. & .- & .- & -- & -- & -- & -- & -. & -. & .. & -- & -- & -- & -- & - & - & -- \\
\hline 8 & - & -- & -- & -- & - & -- & -- & -- & -- & -- & -- & -- & -- & -- & -- & -- & -- \\
\hline 9 & -. & -- & -- & -- & -. & -- & -- & -. & -- & -. & .. & -- & -- & -- & -. & -- & -- \\
\hline 10 & -. & .- & -- & -- & -- & -- & -- & - & -- & -. & -- & -- & -- & -- & -. & .. & -- \\
\hline 11 & .. & -- & -- & -- & - & -- & -- & -- & -- & -- & -- & -- & .- & -- & -. & .- & -- \\
\hline 12 & -. & .. & -- & -- & -. & -- & -- & -- & -- & -- & .. & -- & -- & -- & - & -- & -- \\
\hline 13 & -- & .- & -- & -. & - & -- & -- & -- & -- & -- & -- & -- & -- & -- & -- & -. & .. \\
\hline 14 & -. & -- & -- & - & -- & -- & -- & -- & -- & -- & .. & -- & -- & -- & -- & -- & .- \\
\hline 15 & -- & -- & -- & - & -- & -- & -- & - & -- & -- & -- & -- & -- & -- & -- & -- & -- \\
\hline 16 & .- & -- & .- & -- & - & -- & -- & - & -. & .- & .- & -- & -- & -- & - & -. & -- \\
\hline 17 & -- & -- & -. & -- & -- & -- & -- & -- & -- & -- & -- & -- & -- & -- & -- & -- & -- \\
\hline 18 & -. & .- & -. & -. & - & -- & -- & -- & -- & -- & -. & -- & -- & -- & - & -- & -- \\
\hline 19 & -- & .- & .. & -- & -- & -- & -- & - & -- & .. & -- & -. & -- & -- & .- & -. & -- \\
\hline 20 & -. & -- & -- & -- & -- & -- & -- & -- & - & -- & -. & -- & -- & -- & - & -- & -- \\
\hline 21 & .- & .- & -. & .- & .. & .- & .- & .- & -- & .- & .- & -- & .- & -- & -- & .- & .. \\
\hline 22 & -- & -- & -- & -- & -- & -- & -- & - & -- & -- & -- & -- & -- & -- & -. & -- & -- \\
\hline 23 & -- & -- & -- & -- & - & -- & .- & -- & -- & -- & -- & -. & -- & -- & -- & -- & -- \\
\hline 24 & -. & .- & -- & -- & -- & -- & -- & -. & -. & -- & -- & -- & -. & -- & -- & -. & -- \\
\hline 25 & -- & -- & -- & -- & -- & -- & -- & -- & -- & -- & -- & -- & -- & -- & $\ldots$ & -- & -- \\
\hline 26 & .. & $\ldots$ & -- & -- & -- & -- & -- & -- & -- & .- & .- & -- & -- & -- & -- & -- & .- \\
\hline 27 & -- & -- & -- & -- & -- & -- & -. & -. & -- & -- & -- & -- & -- & -- & -- & -- & -- \\
\hline 28 & -- & -- & -- & -- & $=$ & -. & -- & - & -- & -- & -- & -- & - & -- & -. & -- & - \\
\hline 29 & .- & .- & -- & (c) & - & -- & -- & -. & -. & -. & -- & .- & .- & -- & .. & .- & .- \\
\hline 30 & -- & -- & -- & -- & - & -- & - & -. & -- & -- & -- & -. & - & -- & -- & -- & -- \\
\hline 31 & -. & -- & -- & -. & -- & 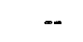 & 0.05 & $=$ & 0.01 & .. & -- & -- & -- & -- & -- & -- & -- \\
\hline Total & 0 & 0 & 0 & (c) & 0 & 0 & 0.05 & 0 & 0.01 & 0 & 0 & 0 & 0 & 0 & 0 & 0 & 0 \\
\hline
\end{tabular}


TABLE 7.--Precipitation data from Weather Service Nuclear Support Office network stations--Continued.

\begin{tabular}{|c|c|c|c|c|c|c|c|c|c|c|c|c|c|c|c|}
\hline \multirow[b]{2}{*}{ DAY } & \multicolumn{15}{|c|}{ Station } \\
\hline & MER & $\mathrm{RV}$ & 4JA & DRA & $\mathrm{CS}$ & W5B & MV & UCC & $40 \mathrm{MN}$ & TS2 & BJY & A12 & PHS & $\mathrm{PM1}$ & $\mathrm{LF} 2$ \\
\hline \multicolumn{16}{|c|}{ JUNE 1984} \\
\hline 1 & - & -- & -- & - & -. & $\ldots$ & -- & $\ldots$ & -- & -- & $-\infty$ & $m$ & $=\infty$ & -- & - \\
\hline 2 & -- & -- & -- & -- & -. & - & -. & -- & - & -. & $\ldots$ & -. & -- & -- & -- \\
\hline 3 & -- & -- & $\cdots$ & $-\infty$ & -- & -- & - & $\infty$ & $\ldots$ & $\cdots$ & $=$ & - & -- & -- & -- \\
\hline 4 & -- & -. & - & -- & -- & -- & -- & -- & -- & -- & -- & - & -- & $\ldots$ & -- \\
\hline 5 & - & -- & - & -- & -- & - & -- & - & $\ldots$ & $\ldots$ & - & $\ldots$ & - & -- & -- \\
\hline 6 & - & -- & - & - & -. & - & -- & -- & -- & -- & -- & -- & -. & - & -- \\
\hline 7 & -- & -. & $\ldots$ & -- & -- & - & -. & -- & -- & -- & -- & -- & - & -- & -- \\
\hline 8 & -- & -. & -- & -- & -- & -- & $-\infty$ & - & - & -- & $\ldots$ & m & -- & -. & -- \\
\hline 9 & -- & -. & - & -- & -- & -- & -- & - & -- & -- & - & - & -- & - & -- \\
\hline 10 & - & -- & -- & - & -- & -- & -. & -. & $=$ & -- & $\ldots$ & $\ldots$ & $\ldots$ & -- & .- \\
\hline 11 & -- & -- & -- & -- & -- & -- & -- & - & -- & -- & -- & -- & -- & -. & .- \\
\hline 12 & -. & -- & -- & $\ldots$ & .- & -- & -. & $\ldots$ & -- & -- & -- & -. & -- & - & -- \\
\hline 13 & - & -- & .- & -- & .. & -- & $\ldots$ & - & - & -- & -- & -- & -- & $-\infty$ & -- \\
\hline 14 & -- & -- & -- & (c) & -- & -- & -- & 0.19 & 0.01 & - & 0.75 & 0.06 & 0.04 & 0.01 & 0.01 \\
\hline 15 & - & -- & -. & - & - & $-\infty$ & $\ldots$ & -- & - & -- & - & -- & -- & -- & - \\
\hline 16 & $\ldots$ & -- & -- & - & -- & -- & -- & -. & -- & .. & -- & -- & -- & - & .. \\
\hline 17 & -- & -- & -- & -- & -- & -- & -. & -- & -. & -- & -- & -. & -. & -- & -- \\
\hline 18 & $-\infty$ & -. & -- & -- & -. & -- & $-\infty$ & 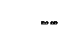 & -- & - & -- & - & -- & - & -- \\
\hline 19 & -- & -. & -- & - & -- & -. & $\ldots$ & -- & -- & -- & -- & $\ldots$ & -- & -- & -- \\
\hline 20 & -- & -- & -- & - & -- & -- & -. & -- & $-\infty$ & -. & -- & -- & -- & $\infty$ & -. \\
\hline 21 & -- & -. & -- & -- & -- & - & -. & -- & -. & -- & -- & $=$ & -- & - & -- \\
\hline 22 & -- & -- & -- & -- & -- & -- & -. & -- & $\ldots$ & -- & -- & -- & -. & $\ldots$ & .- \\
\hline 23 & -- & .. & -- & -- & -- & - & .. & -- & -- & -- & - & - & - & - & -. \\
\hline 24 & 0.02 & -. & $\ldots$ & (c) & -- & -- & -. & -. & $\ldots$ & -- & -- & -. & - & - & -- \\
\hline 25 & - & -- & - & $=$ & -. & -- & -- & -- & $=$ & - & -. & -. & - & -- & -. \\
\hline 26 & -- & -- & -- & - & .. & $=$ & -- & -- & -- & - & - & -- & -. & -- & .. \\
\hline 27 & -. & -- & $\ldots$ & -- & .- & -- & -- & -. & -- & -- & -. & -- & -. & - & .. \\
\hline 28 & -- & -- & -. & - & .- & - & $\ldots$ & .. & -- & .. & $-\infty$ & .- & $\ldots$ & -- & -. \\
\hline 29 & -- & -- & -- & -- & -- & -- & -. & -- & -- & -- & - & -- & -- & -- & -- \\
\hline 30 & -- & -- & - & - & -- & - & $-\infty$ & - & -. & -- & - & -- & $\ldots$ & -- & -. \\
\hline Total & 0.02 & 0 & 0 & (c) & 0 & 0 & 0 & 0.19 & 0.01 & 0 & 0.75 & 0.06 & 0.04 & 0.01 & 0.01 \\
\hline
\end{tabular}


TABLE 7.--Precipitation data from Weather Service Nuclear Support Office nework stations--Continued.

\begin{tabular}{|c|c|c|c|c|c|c|c|c|c|c|c|c|c|c|c|}
\hline \multirow[b]{2}{*}{ DAY } & \multicolumn{15}{|c|}{ Station } \\
\hline & MER & $\mathbf{R V}$ & $4 J \mathrm{~A}$ & DRA & $\mathrm{CS}$ & W5B & MV & UCC & $40 \mathrm{MN}$ & TS2 & BJY & $\mathrm{A} 12$ & PHS & PM1 & $\mathrm{LF} 2$ \\
\hline \multicolumn{16}{|c|}{ JULY 1984} \\
\hline 1 & $=$ & $=$ & -- & -- & $=$ & $=$ & - & - & - & - & -- & - & - & -. & - \\
\hline 2 & 0.10 & -- & $\cdots$ & 0.19 & -- & - & - & 0.02 & - & 0.02 & - & 0.44 & -- & 0.30 & 0.35 \\
\hline 3 & -- & -- & 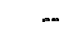 & .02 & $\infty$ & -- & $-\infty$ & - & 0.03 & .17 & $\infty$ & - & 0.04 & -. & -- \\
\hline 4 & $-\infty$ & $-\infty$ & -- & -- & $\cdots$ & - & - & -- & - & - & - & - & -- & - & -- \\
\hline 5 & -- & - & $\cdots$ & - & - & -- & - & - & - & - & $-\infty$ & -- & -- & -- & - \\
\hline 6 & -- & -- & - & $\cdots$ & - & - & - & - & - & - & - & - & - & - & -- \\
\hline 7 & -- & $-\cdots$ & - & $=$ & - & - & - & $-\infty$ & $-\infty$ & -- & - & -- & -- & -- & - \\
\hline 8 & $=$ & -- & $=$ & -- & $=$ & $=$ & -- & $-\infty$ & - & $-\infty$ & -- & $\cdots$ & 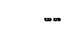 & $=$ & -- \\
\hline 9 & -- & -- & -- & $\cdots$ & $\cdots$ & - & -- & -- & -- & - & -- & - & - & $\cdots$ & -- \\
\hline 10 & - & $\cdots$ & $=$ & $\infty$ & -- & - & $\cdots$ & $-\infty$ & $=$ & - & $-\cdots$ & - & - & -- & $\cdots$ \\
\hline 11 & -- & -- & - & - & - & - & - & -- & -- & - & -- & -- & -- & - & - \\
\hline 12 & -- & -. & -. & (c) & -- & - & -- & - & (b) & - & - & .01 & -- & .06 & - \\
\hline 13 & .12 & (b) & 0.05 & .13 & -. & 0.07 & $\infty$ & $\infty$ & (b) & .05 & -- & .05 & .14 & - & (b) \\
\hline 14 & -- & (b) & -- & -- & -- & -- & $-\infty$ & -. & (b) & -- & - & .01 & - & -- & (b) \\
\hline 15 & 1.20 & (b) & $\cdots$ & - & -- & - & -- & .01 & (b) & $\ldots$ & 0.03 & .10 & .05 & 1.12 & (b) \\
\hline 16 & .10 & (b) & - & .03 & -- & $\infty$ & 0.12 & (b) & $f_{.47}$ & -- & - & .03 & -- & -- & (b) \\
\hline 17 & -- & (b) & $=$ & $\infty$ & $=$ & - & -. & (b) & .04 & .03 & .12 & -. & - & .01 & $f .36$ \\
\hline 18 & $-\infty$ & (b) & - & -- & $-\infty$ & .03 & $=$ & (b) & .06 & -. & .01 & .09 & .03 & - & -- \\
\hline 19 & .07 & (b) & .01 & .19 & 0.59 & .22 & .12 & (b) & .01 & .01 & .07 & .64 & .01 & 1.50 & .02 \\
\hline 20 & .08 & (b) & - & .14 & .01 & .01 & $=$ & (b) & =- & - & - & - & - & .24 & -- \\
\hline 21 & .20 & (b) & .31 & .32 & 1.00 & .52 & .46 & (b) & 1.06 & 1.23 & 1.32 & .90 & 1.37 & .06 & 1.71 \\
\hline 22 & 2.38 & (b) & 2.47 & 2.03 & 1.36 & 1.64 & 1.67 & (b) & 1.27 & 1.68 & .93 & .75 & 1.00 & (b) & .52 \\
\hline 23 & .03 & (b) & .01 & .20 & - & $=$ & -- & (b) & -- & -- & - & - & -- & (b) & -- \\
\hline 24 & - & (b) & .01 & -- & -- & - & - & $f_{1.72}$ & -- & -- & -- & - & - & (b) & -- \\
\hline 25 & - & (b) & - & - & - & -- & .02 & 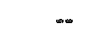 & - & - & -- & -- & - & (b) & - \\
\hline 26 & - & (b) & - & -- & $\infty$ & $-\infty$ & -- & -- & - & -- & - & .18 & - & (b) & - \\
\hline 27 & .06 & (b) & - & .03 & .16 & .03 & .22 & .49 & - & .04 & .65 & .04 & 1.27 & (b) & -- \\
\hline 28 & .02 & (b) & .18 & .03 & .04 & - & .07 & .04 & .14 & .13 & .07 & .26 & .04 & (b) & .20 \\
\hline 29 & -- & (b) & .07 & - & - & - & .02 & -- & .37 & .05 & -- & .01 & -- & (b) & .07 \\
\hline 30 & -- & $f_{2.83}$ & .06 & -. & -- & - & .03 & - & .13 & .02 & .. & .07 & .12 & (b) & .78 \\
\hline 31 & .04 & .29 & 1.33 & .33 & .18 & .10 & .13 & .79 & .06 & .03 & .22 & .19 & .01 & $f_{1.11}$ & .03 \\
\hline Total & 4.40 & 3.12 & 4.50 & 3.64 & 3.34 & 2.62 & 2.86 & 3.07 & 3.64 & 3.46 & 3.42 & 3.77 & 4.08 & 4.40 & 4.04 \\
\hline
\end{tabular}


TABLE 7.--Precipitation data from Weather Service Nuclear Support Office nework stations--Continued.

\begin{tabular}{|c|c|c|c|c|c|c|c|c|c|c|c|c|c|c|c|}
\hline \multirow[b]{2}{*}{ DAY } & \multicolumn{15}{|c|}{ Station } \\
\hline & MER & RV & $4 \mathrm{JA}$ & DRA & $\mathrm{CS}$ & W5B & MV & $\mathrm{UCC}$ & $40 \mathrm{MN}$ & $T S 2$ & B.JY & A12 & PHS & PM1 & $\mathrm{LF}_{2}$ \\
\hline \multicolumn{16}{|c|}{ AUGUST 1984} \\
\hline 1 & - & $\cdots$ & $=$ & $-\infty$ & -- & $m$ & - & $\infty$ & $-\infty$ & -- & - & - & $-\infty$ & $(a)$ & -- \\
\hline 2 & -- & - & -- & -- & $-\infty$ & -- & -- & -- & - & - & - & - & - & (a) & -- \\
\hline 3 & -- & $-\infty$ & $-\infty$ & $\infty$ & $-\infty$ & - & $-\infty$ & $m$ & $-\infty$ & -- & $-\infty$ & - & $\ldots$ & (a) & - \\
\hline 4 & $\ldots$ & -. & -- & -- & -- & - & $-\infty$ & -- & $\ldots$ & - & - & -- & - & (a) & -. \\
\hline 5 & -- & - & - & - & - & -- & $-\infty$ & $\infty$ & - & - & - & $\ldots$ & - & (a) & -- \\
\hline 6 & - & - & 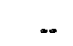 & -. & - & $\ldots$ & - & $-\infty$ & - & - & - & $=$ & -- & (a) & $\infty$ \\
\hline 7 & -- & - & - & -- & - & -- & -- & - & - & -- & -- & -- & -- & (a) & -- \\
\hline 8 & -. & -- & -- & $-\infty$ & - & -- & $\ldots$ & $\infty$ & -- & $\cdots$ & - & $=$ & -. & (a) & .. \\
\hline 9 & -- & - & - & -- & - & - & - & -. & -. & $=$ & - & -- & .- & (a) & -- \\
\hline 10 & -. & .07 & -. & (c) & - & - & -. & -- & - & $\ldots$ & - & .01 & -- & (b) & -- \\
\hline 11 & -- & - & - & (c) & .03 & .01 & -- & -. & .07 & -. & -- & .04 & $=$ & (b) & .05 \\
\hline 12 & $-\infty$ & -- & -. & - & -- & $\ldots$ & $-\infty$ & - & - & - & - & -- & -- & (b) & -- \\
\hline 13 & .. & -. & $-\infty$ & -. & $\ldots$ & $=$ & -. & -. & .. & -. & -- & -. & -. & (b) & .. \\
\hline 14 & .71 & .96 & .70 & .94 & .53 & .66 & .89 & 1.52 & .77 & .85 & 1.50 & .56 & .64 & (b) & .54 \\
\hline 15 & .59 & .38 & .13 & 1.02 & (b) & .55 & .58 & .18 & 1.14 & .31 & .27 & .40 & .19 & (b) & .75 \\
\hline 16 & -- & (b) & .01 & -- & (b) & $\ldots$ & .04 & .01 & .01 & -- & .01 & .02 & (b) & (b) & .01 \\
\hline 17 & -- & (b) & - & -. & (b) & -. & .01 & .01 & .01 & -. & .01 & .13 & (b) & (b) & .01 \\
\hline 18 & .20 & (b) & .11 & .16 & (b) & .20 & .76 & .64 & .17 & .90 & .51 & .07 & (b) & (b) & .15 \\
\hline 19 & .98 & (b) & .79 & 1.02 & (b) & .75 & .75 & .79 & 1.02 & .34 & .69 & .30 & (b) & (b) & .61 \\
\hline 20 & -- & $f .69$ & - & $=$ & $f_{1.37}$ & - & $\infty$ & -- & .05 & - & .02 & .09 & (b) & (b) & .05 \\
\hline 21 & -- & - & - & - & - & - & -- & - & .03 & - & - & .01 & (b) & (b) & .02 \\
\hline 22 & $\ldots$ & - & - & - & m & - & - & $-\infty$ & - & $\infty$ & -. & - & (b) & (b) & -- \\
\hline 23 & $=$ & $\ldots$ & $\infty$ & -. & $\infty$ & - & $\ldots$ & - & $-\infty$ & -- & $\ldots$ & - & (b) & (b) & -- \\
\hline 24 & $\cdots$ & -- & -- & -. & - & -- & $\ldots$ & $\ldots$ & - & - & -- & - & (b) & (b) & -- \\
\hline 25 & $-\infty$ & $-\infty$ & - & (c) & -. & .36 & - & - & - & -. & .03 & .18 & $f_{1.22}$ & (b) & - \\
\hline 26 & -- & .. & $\ldots$ & - & .. & .. & - & .16 & -. & -. & $\cdots$ & - & $\ldots$ & $f_{4.09}$ & -- \\
\hline 27 & - & -- & - & - & - & - & -- & -. & -- & - & -- & -- & - & (a) & - \\
\hline 28 & -. & - & .. & - & -. & $\infty$ & - & -. & $\ldots$ & - & -- & $\ldots$ & - & (a) & .. \\
\hline 29 & $\ldots$ & -- & -- & - & -. & - & -- & - & -- & -- & - & -- & - & (a) & - \\
\hline 30 & $=$ & -. & -. & -. & $=$ & - & $m$ & $a$ & -- & - & $\infty$ & $\infty$ & $\infty$ & (a) & .. \\
\hline 31 & - & $=\infty$ & - & - & -. & - & - & -- & - & - & -- & - & - & (a) & - \\
\hline Total & 2.48 & 2.10 & 1.74 & 3.14 & 1.93 & 2.53 & 3.03 & 3.31 & 3.27 & 2.40 & 3.04 & 1.81 & 2.05 & 4.09 & 2.19 \\
\hline
\end{tabular}


TABLE 7.--Precipitation data from Weather Service Nuclear Support Office nework stations--Continued.

Station

\begin{tabular}{|c|c|c|c|c|c|c|c|c|c|c|c|c|c|c|c|}
\hline DAY & MER & RV & 4JA & DRA & $\mathrm{CS}$ & W5B & MV & UCC & $40 \mathrm{MN}$ & $\mathrm{TS} 2$ & BJY & A12 & PHS & PM1 & $\mathrm{LF} 2$ \\
\hline \multicolumn{16}{|c|}{ SEPTEMBER 1984} \\
\hline 1 & -- & -. & .- & .. & .. & .- & -- & .. & .. & .- & -- & -. & .- & (a) & .. \\
\hline 2 & -- & -- & -- & - & -. & -- & -- & -- & -. & - & -. & -- & -- & (a) & -- \\
\hline 3 & -- & .. & .. & -. & .. & -- & .- & .- & -- & -- & $\ldots$ & .- & .. & (a) & .. \\
\hline 4 & -- & -- & -- & -- & -. & -- & -- & - & -- & -- & _- & -- & -. & (a) & -- \\
\hline 5 & -- &.- & -. & - & -- & -- & -- & -- & -- & - & -- & - & -- & (a) & - \\
\hline 6 & -- & -. & $\ldots$ & -- & -- & -- & -. & .. & -- & -- & -. & - & -. & -- & .. \\
\hline 7 & -- & -- & -- & -- & -- & -- & - & -. & -- & -- & -- & - & $\ldots$ & -. & -- \\
\hline 8 & - & -- & - & - & -- & -. & - & -- & -. & -- & -. & - & - & .. & -- \\
\hline 9 & - & -. & -- & -- & -- & -- & - & -- & -- & $-\infty$ & -- & - & -- & -- & -- \\
\hline 10 & .. & -- & .. & (c) & -. & -- & -- & -- & -- & -- & -- & 0.10 & 0.02 & 0.10 & -- \\
\hline 11 & - & - & -- & -. & $\ldots$ & -- & -- & - & -. & -- & -- & - & -. & .. & -. \\
\hline 12 & -- & .- & .- & (c) & -- & -- & -- & $\ldots$ & -. & -- & -- & -- & -- & .- & -- \\
\hline 13 & -- & -- & -- & $\ldots$ & -- & -- & -- & $\ldots$ & -- & -- & -. & -- & -- & -- & -- \\
\hline 14 & $\ldots$ & -- & .- & -- & -. & -- & .- & -- & -. & -- & -- & -. & $\ldots$ & -- & -- \\
\hline 15 & - & 0.07 & - & 0.01 & 0.07 & -- & - & 0.01 & -- & -- & -- & .. & -. & -. & -- \\
\hline 16 & $\infty$ & .03 & .- & (c) & .03 & 0.10 & .. & .04 & 1.29 & 0.05 & 0.03 & .18 & .01 & -. & .- \\
\hline 17 & -- & -- & 0.10 & -- & - & -- & .- & -. & .03 & .01 & .. & .04 & - & - & -- \\
\hline 18 & -. & .. & -. & -- & -. & -- & .. & - & -- & -. & .- & .32 & .14 & -. & 0.04 \\
\hline 19 & $\ldots$ & .03 & .- & -- & -- & -- & -- & -- & -- & -- & -- & -- & .08 & -- & -- \\
\hline 20 & $\ldots$ & - & .20 & -- & - & -- & .- & -- & -- & -. & -- & -- & - & -. & -- \\
\hline 21 & -- & -- & .- & -. & -. & ... & .- & .. & -. & -- & -- & .. & $\ldots$ & -. & -.- \\
\hline 22 & - & .- & -- & -- & -- & -- & -- & -. & -. & -- & -- & -- & -- & -. & -- \\
\hline 23 & -- & -- & -. & - & -- & -- & -- & -- & -. & $\ldots$ & -- & -- & -- & -. & .- \\
\hline 24 & -- & -- & -- & -- & - & - & -- & -- & -. & -- & .- & -- & -- & - & -- \\
\hline 25 & -- & -- & -.. & -. & -- & .. & -- & -. & -- & -- & -. & -- & - & $=$ & .. \\
\hline 26 & -. & -- & -- & -- & - & -. & -- & -- & - & - & -- & -- & - & - & -- \\
\hline 27 & .. & -- & -- & . & ... & -- & -- & -- & -. & -- & -- & -- & - & -. & .- \\
\hline 28 & .- & -- & -- & -- & - & $\ldots$ & -. & -- & -- & -- & .- & .- & -- & - & -. \\
\hline 29 & -- & .- & .- & -- & -. & -- & -. & -. & -- & -- & -- & .. & - & -- & .- \\
\hline 30 & -- & -- & - & -. & - & -- & -- & $-\infty$ & -- & -- & - & .02 & - & -. & .- \\
\hline Total & 0 & 0.13 & 0.30 & 0.01 & 0.10 & 0.10 & 0 & 0.05 & 1.32 & 0.06 & 0.03 & 0.66 & 0.25 & 0.10 & 0.04 \\
\hline
\end{tabular}


TABLE 7.--Precipitation data from Weather Service Nuclear Support Office network stations--Continued.

\begin{tabular}{|c|c|c|c|c|c|c|c|c|c|c|c|c|c|c|c|}
\hline \multirow[b]{2}{*}{ DAY } & \multicolumn{15}{|c|}{ Station } \\
\hline & MER & RV & $4 \mathrm{JA}$ & DRA & $\mathrm{CS}$ & W5B & MV & UCC & $40 \mathrm{MN}$ & $\mathrm{TS} 2$ & BJY & A12 & PHS & PMI & $\mathrm{LF} 2$ \\
\hline \multicolumn{16}{|c|}{ OCTOBER 1984} \\
\hline 1 & 0.14 & 0.11 & 0.01 & 0.12 & 0.14 & 0.20 & 0.06 & 0.01 & 0.07 & 0.04 & 0.04 & 0.10 & 0.11 & 0.04 & 0.01 \\
\hline 2 & -. & .01 & (b) & .02 & .03 & .07 & .21 & .15 & .32 & .20 & .13 & .12 & .22 & .11 & .32 \\
\hline 3 & - & -. & (b) & -- & - & -- & -. & -. & -- & $\infty$ & - & -- & -- & -. & -- \\
\hline 4 & -- & -- & (b) & -- & -- & -. & -. & -- & -- & -- & - & - & -- & -. & -. \\
\hline 5 & -- & - & (b) & -- & - & - & -- & - & $-\infty$ & - & - & - & - & -- & - \\
\hline 6 & -- & -. & (b) & -- & -- & -. & .. & -. & -- & .. & -- & -- & -- & -- & -. \\
\hline 7 & - & -- & (b) & -- & -- & -. & .. & -- & -- & .. & - & - & -- & -- & -. \\
\hline 8 & - & -- & (b) & - & ... & -- & .. & -.. & $\ldots$ & -- & -- & $\ldots$ & $-\infty$ & -- & -- \\
\hline 9 & -. & - & (b) & - & -- & -. & -. & -- & -- & -- & -- & -- & .- & -- & -- \\
\hline 10 & - & -- & (b) & -. & -. & -- & -- & -. & -. & $\ldots$ & - & - & -. & -- & -- \\
\hline 11 & -- & -- & (b) & - & -- & $-\infty$ & -- & -- &.- & -- & -- & .01 & .02 & -. & -- \\
\hline 12 & - & -- & (b) & - & .- & -- & -- & -- & -. & $\ldots$ & - & -. & -- & -.. & $\ldots$ \\
\hline 13 & -- & $\infty$ & (b) & $-\infty$ & -- & -- & -- & $\ldots$ & $\ldots$ & -- & -- & $-=$ & - & -. & -- \\
\hline 14 & -- & -- & (b) & -- & -- & -. & -- & - & -. & -- & -- & -- & -- & -. & -- \\
\hline 15 & - & -- & (b) & $m$ & $\infty$ & - & -- & -- & -. & - & - & -- & -- & -- & -- \\
\hline 16 & -- & $\ldots$ & (b) & -. & -- & -- & -- & - & -- & -- & -- & - & - & .01 & -- \\
\hline 17 & -- & $\ldots$ & (b) & -. & -- & -- & -- & - & -- & -- & -- & - & -- & -- & -- \\
\hline 18 & - & -. & (b) & -- & -- & .. & -- & .. & -- & -- & -- & -. & -. & -. & -- \\
\hline 19 & -- & .. & (b) & -- & -- & -- & -- & -- & -- & -- & -- & -- & -- & -- & -- \\
\hline 20 & - & .02 & (b) & .01 & -. & - & -- & - & -- & .01 & .04 & .12 & .01 & .02 & -- \\
\hline 21 & -- & .01 & $f_{.02}$ & - & -- & -. & -- & -. & - & -- & - & - & -- & -- & -- \\
\hline 22 & -- & .. & (a) & -- & -- & -- & - & -- & -- & -- & -- & - & -. & -- & -- \\
\hline 23 & - & $-\infty$ & (a) & -- & -- & -. & -- & m. & -- & -- & -- & $=$ & .- & -- & -- \\
\hline 24 & - & -- & (a) & -- & -- & -. & -- & -- & -. & -- & -- & -. & .. & -- & -- \\
\hline 25 & $=$ & -- & (a) & -- & -. & $\ldots$ & -- & -. & -- & -- & -- & $=$ & $\ldots$ & -- & -- \\
\hline 26 & $\infty$ & -- & (a) & - & -- & -- & -- & -. & - & - & - & -. & -- & -- & -. \\
\hline 27 & -- & -- & (a) & $\ldots$ & -. & .. & -- & -. & $\ldots$ & - & - & -- & -- & -- & -- \\
\hline 28 & - & -. & (a) & - & $-\infty$ & $=$ & -- & $=0$ & $\infty$ & $\infty$ & - & $=$ & - & - & -. \\
\hline 29 & -- & -. & (a) & -. & -. & -- & - & -. & $\ldots$ & -- & -- & -- & -- & -- & -- \\
\hline 30 & - & $\ldots$ & (a) & - & -- & -- & -- & -. & -. & -- & -- & -. & - & -- & -. \\
\hline 31 & -- & -- & (a) & -- & -- & -. & - & -. & -- & - & -- & -- & -. & -- & -- \\
\hline Total & 0.14 & 0.15 & 0.03 & 0.15 & 0.17 & 0.27 & 0.27 & 0.16 & 0.39 & 0.25 & 0.21 & 0.35 & 0.36 & 0.18 & 0.33 \\
\hline
\end{tabular}


TABLE 7.--Precipitation data from Weather Senice Nuclear Support Office network stations--Continued.

\begin{tabular}{|c|c|c|c|c|c|c|c|c|c|c|c|c|c|c|c|}
\hline \multirow[b]{2}{*}{ DAY } & \multicolumn{15}{|c|}{ Station } \\
\hline & $\mathrm{MER}$ & RV & 4JA & DRA & $\mathrm{CS}$ & W5B & MV & $\mathrm{UCC}$ & $40 \mathrm{MN}$ & $\mathrm{T} \$ 2$ & BIY & $\mathrm{A} 12$ & PHS & PM1 & $\mathrm{LF} 2$ \\
\hline \multicolumn{16}{|c|}{ NOVEMBER 1984} \\
\hline 1 & -. & $\ldots$ & (a) & - & -- & -- & -- & -- & -- & - & - & 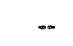 & - & -- & -- \\
\hline 2 & - & -- & (a) & -- & - & $-\infty$ & - & $-\infty$ & $\ldots$ & $-\infty$ & - & -- & - & $-\infty$ & $-\infty$ \\
\hline 3 & $\ldots$ & $=$ & (a) & $=$ & - & $\infty$ & - & $-\infty$ & $\cdots$ & -- & -- & 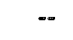 & $=$ & - & - \\
\hline 4 & - & $-\cdots$ & (a) & $-\infty$ & - & -- & -- & -- & -- & -- & -- & -- & - & -- & -- \\
\hline 5 & $-\cdots$ & -- & (a) & $-\infty$ & - & $\cdots$ & - & $\approx$ & $=$ & $=$ & - & $=$ & $\infty$ & $\infty$ & - \\
\hline 6 & -- & $-\infty$ & $\cdots$ & $\ldots$ & - & - & - & -- & -- & - & - & $\ldots$ & - & - & -- \\
\hline 7 & -- & -- & $\ldots$ & -- & - & -- & -- & $=$ & $-\infty$ & - & - & -. & $\cdots$ & -- & -- \\
\hline 8 & - & - & - & (c) & -- & $-\infty$ & 0.02 & $-\infty$ & - & 0.02 & - & 0.01 & $\ldots$ & $=$ & $=$ \\
\hline 9 & - & -- & - & -- & - & -- & -- & - & -- & -- & -- & -- & -- & - & -- \\
\hline 10 & -- & $=$ & - & - & - & -- & -- & - & $\cdots$ & -- & - & $-\cdots$ & - & - & - \\
\hline 11 & - & - & - & -- & -- & - & $-\infty$ & - & -- & -- & - & $\ldots$ & $-\infty$ & - & -- \\
\hline 12 & - & - & -- & -- & -- & $-\infty$ & - & - & -- & - & - & -. & -. & - & $=-$ \\
\hline 13 & $\ldots$ & - & - & (c) & - & -- & .01 & - & 0.02 & .02 & $-\infty$ & .06 & $\infty$ & - & $-\infty$ \\
\hline 14 & $\cdots$ & - & - & -- & -- & - & -- & -- & - & - & - & -- & -- & -- & -- \\
\hline 15 & $=$ & - & - & -- & $=$ & $-\infty$ & $\cdots$ & - & $=$ & $=$ & $-\infty$ & - & $\cdots$ & -- & $=$ \\
\hline 16 & $-\infty$ & -- & $\ldots$ & - & - & -- & - & - & - & -- & -- & -- & -- & - & -- \\
\hline 17 & -- & - & $-\infty$ & - & $=$ & $\infty$ & - & - & -- & $-\infty$ & $\ldots$ & -- & - & - & - \\
\hline 18 & -- & $\alpha=$ & $\infty$ & (c) & - & - & -- & $\ldots$ & - & - & 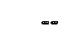 & - & $\ldots$ & - & -- \\
\hline 19 & - & -- & - & - & -- & -- & -- & $\cdots$ & - & - & - & - & $\ldots$ & - & -- \\
\hline 20 & $=$ & $\cdots$ & - & - & $=$ & - & - & a & $=$ & - & - & - & $\cdots$ & -- & - \\
\hline 21 & 0.07 & 0.09 & 0.09 & 0.05 & 0.08 & 0.03 & .06 & 0.01 & .13 & .03 & 0.02 & .09 & - & (b) & 0.08 \\
\hline 22 & 1.16 & .95 & (b) & 1.48 & 1.18 & 1.00 & 1.15 & (b) & (b) & .76 & .59 & .93 & 0.64 & (b) & 1.14 \\
\hline 23 & .01 & .01 & (b) & .01 & .08 & (b) & .08 & (b) & (b) & .15 & .02 & .40 & .02 & (b) & .05 \\
\hline 24 & .10 & .04 & (b) & .22 & .18 & (b) & .12 & (b) & (b) & .21 & .20 & .42 & .14 & (b) & (b) \\
\hline 25 & .25 & -- & $f .90$ & $(c)$ & .01 & $f_{.22}$ & .09 & $f_{1.16}$ & $f_{1.22}$ & .05 & .07 & - & .03 & $f_{1.22}$ & $f .15$ \\
\hline 26 & - & -- & -- & -- & -- & - & - & -- & -- & - & - & -- & - & - & - \\
\hline 27 & - & $=$ & - & - & $-\infty$ & -- & -- & $\infty$ & -. & $=$ & -- & $=$ & -- & -. & - \\
\hline 28 & 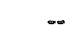 & - & - & - & - & - & - & -- & -- & - & - & - & -- & - & -- \\
\hline 29 & -- & -- & - & - & -- & -- & - & $-\infty$ & -. & -- & -- & $=$ & - & -- & - \\
\hline 30 & $=$ & $=$ & -- & - & - & $\infty$ & - & - & -. & - & -- & - & - & - & -- \\
\hline Total & 1.59 & 1.09 & 0.99 & 1.76 & 1.53 & 1.25 & 1.53 & 1.17 & 1.37 & 1.24 & 0.90 & 1.91 & 0.83 & 1.22 & 1.42 \\
\hline
\end{tabular}


TABLE 7.--Precipitation data from Weather Service Nuclear Support Office network stations--Continued.

\begin{tabular}{|c|c|c|c|c|c|c|c|c|c|c|c|c|c|c|c|}
\hline \multirow[b]{2}{*}{ DAY } & \multicolumn{15}{|c|}{ Station } \\
\hline & MER & RV & 4JA & DRA & $\mathrm{CS}$ & W5B & MV & UCC & $40 \mathrm{MN}$ & $\mathrm{TS} 2$ & BJY & A12 & PHS & PM1 & $\mathrm{LF}_{2}$ \\
\hline \multicolumn{16}{|c|}{ DECEMBER 1984} \\
\hline 1 & - & -- & -- & -- & -- & - & - & -- & -- & -- & -- & - & -- & -- & -- \\
\hline 2 & - & -- & -- & -- & - & -- & - & -- & - & -- & - & -- & -. & - & -- \\
\hline 3 & -- & -- & -- & -- & -- & -- & -- & -- & -- & -- & -- & -- & $\ldots$ & -- & -. \\
\hline 4 & -- & -- & (a) & - & -- & -. & -- & -- & -- & -- & -- & -- & -- & -- & -- \\
\hline 5 & - & -- & (a) & - & -- & -- & - & -- & -- & - & -- & -- & -- & - & -- \\
\hline 6 & - & - & (a) & -- & - & .- & - & $-\infty$ & -- & -- & -- & -. & .- & -- & -- \\
\hline 7 & -- & -- & (a) & -- & -- & -- & -- & -. & -. & -- & -- & -- & -- & -- & -- \\
\hline 8 & 0.05 & 0.08 & (b) & 0.06 & 0.22 & 0.20 & 0.07 & (b) & 0.01 & 0.05 & 0.10 & 0.04 & 0.04 & - & -- \\
\hline 9 & -. & -- & (b) & - & -. & -- & -- & (b) & -- & -- & .. & -. & -- & -- & -. \\
\hline 10 & -- & .02 & (b) & (c) & .02 & -- & .16 & $f_{0.18}$ & .01 & .03 & .03 & .07 & .01 & 0.24 & 0.02 \\
\hline 11 & - & .01 & (b) & - & .01 & -- & .04 & .03 & .16 & .05 & .02 & .09 & .03 & .04 & .09 \\
\hline 12 & .11 & .04 & (b) & .06 & .01 & .10 & .14 & .04 & -- & - & .03 & .06 & .01 & - & $\ldots$ \\
\hline 13 & -- & -. & (b) & - & .02 & - & -- & $=$ & -- & - & -. & -- & -- & .01 & -- \\
\hline 14 & .- & -- & (b) & -- & - & -- & -. & -- & -. & -- & -- & -- & -- & -- & -- \\
\hline 15 & $=-$ & .01 & (b) & (c) & .06 & -- & .15 & .01 & .10 & .32 & .05 & .28 & $\ldots$ & (b) & .20 \\
\hline 16 & .02 & .21 & (b) & .02 & .35 & .14 & .59 & .15 & .32 & .45 & .24 & .62 & .17 & (b) & .27 \\
\hline 17 & - & -. & (b) & - & -. & -- & .02 & .01 & .03 & -- & -. & .05 & - & (b) & - \\
\hline 18 & .18 & .06 & (b) & .44 & .44 & .29 & .36 & .27 & .14 & .39 & .32 & .28 & .03 & (b) & .33 \\
\hline 19 & .77 & 1.20 & (b) & .65 & 1.08 & .84 & 1.09 & 1.13 & .67 & 1.17 & .95 & (b) & .54 & (b) & .58 \\
\hline 20 & .07 & .01 & (b) & (c) & .06 & .02 & .14 & .16 & .05 & .07 & .14 & (b) & .38 & (b) & .02 \\
\hline 21 & -- & -- & (b) & - & .. & - & -- & -. & .02 & -- & -. & (b) & .. & (b) & -- \\
\hline 22 & -. & .- & (b) & -- & - & -- & -- & -- & -. & -- & - & (b) & -- & (b) & .- \\
\hline 23 & -- & -- & (b) & -- & .- & -- & .- & -- & $\ldots$ & - & -- & (b) & .03 & (b) & -- \\
\hline 24 & -- & .. & (b) & -. & -. & -- & -- & -- & -- & - & -- & (b) & .05 & (b) & -. \\
\hline 25 & -- & -- & (b) & -- & - & -- & -- & -- & $\ldots$ & -- & -- & (b) & .07 & (b) & - \\
\hline 26 & .21 & .21 & (b) & .33 & .16 & .15 & (b) & .06 & .03 & .02 & .03 & (b) & -- & (b) & .02 \\
\hline 27 & .22 & .27 & (b) & .25 & .41 & .33 & (b) & .38 & .34 & .38 & .37 & (b) & .25 & (b) & .21 \\
\hline 28 & .13 & .23 & (b) & .10 & .28 & .17 & (b) & .32 & .18 & (b) & .15 & (b) & .11 & (b) & .24 \\
\hline 29 & - & - & $f_{2.43}$ & -- & .01 &. & $f_{.76}^{10}$ & - & .02 & (b) & - & (b) & - & (b) & .02 \\
\hline 30 & - & -- & (a) & $\ldots$ & - & $-\infty$ & -- & - & $-\infty$ & ${ }^{d} .23$ & -- & $d_{.42}$ & .01 & $f .85$ & - \\
\hline 31 & -. & -- & (a) & -- & -. & -- & -. & -- & -. & (a) & -- & (a) & -- & (a) & - \\
\hline Total & 1.76 & 2.35 & 2.43 & 1.91 & 3.13 & 2.24 & 3.52 & 2.74 & 2.08 & $e_{3.16}$ & 2.43 & $e_{1.91}$ & 1.73 & 1.14 & 2.00 \\
\hline
\end{tabular}


TABLE 7.--Precipitation data from Weather Service Nuclear Support Office network stations--Continued.

\begin{tabular}{|c|c|c|c|c|c|c|c|c|c|c|c|c|c|c|c|}
\hline \multirow[b]{2}{*}{ DAY } & \multicolumn{15}{|c|}{ Station } \\
\hline & MER & RV & $4 J \mathrm{~A}$ & DRA & $\mathrm{CS}$ & W5B & MV & UCC & $40 \mathrm{MN}$ & $\mathrm{TS} 2$ & BJY & $\mathrm{Al}$ & 2 PHS & S PM. & LF2 \\
\hline \multicolumn{16}{|c|}{ JANUARY 1985} \\
\hline 1 & - & - & (a) & -- & -- & -- & -- & -- & -. & (a) & - & (a) & -- & (a) & $\ldots$ \\
\hline 2 & -- & - & (a) & - & $=$ & $\ldots$ & -- & $-\infty$ & -- & (a) & -- & (a) & - & (a) & -- \\
\hline 3 & -- & 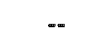 & (a) & - & - & -. & - & -- & $\ldots$ & (a) & $\ldots$ & (a) & - & (a) & $=$ \\
\hline 4 & -. & .. & (a) & -- & -- & ... & -. & -.- & -- & (a) & -- & (a) & -- & (a) & .- \\
\hline 5 & -- & $-\infty$ & (a) & -- & - & -- & -- & - & -- & (a) & - & (a) & - & (a) & - \\
\hline 6 & -- & -- & (a) & -- & -- & -- & $\ldots$ & - & -- & (a) & - & (a) & - & (a) & -- \\
\hline 7 & 0.41 & 0.39 & (b) & 0.68 & 0.52 & 0.30 & 0.62 & 0.64 & 0.37 & (b) & 0.46 & (b) & 0.25 & (b) & 0.27 \\
\hline 8 & .04 & .54 & $d_{0.48}$ & .10 & .14 & .17 & .04 & .23 & .02 & (b) & .02 & (b) & .07 & (b) & (b) \\
\hline 9 & - & (a) & (a) & - & - & -. & - & - & $.02 d$ & 0.23 & $-d_{0}$ & 0.83 & $-g$ & $8_{0.50}$ & $f_{.08}$ \\
\hline 10 & $=$ & (a) & (a) & -. & - & -- & -- & -- & -. & (a) & -- & (a) & -- & (a) & (a) \\
\hline 11 & - & (a) & (a) & -- & -- & -. & -. & - & -- & (a) & -- & (a) & - & (a) & (a) \\
\hline 12 & $\ldots$ & (a) & (a) & $\ldots$ & $=$ & -- & $\ldots$ & $\ldots$ & -. & (a) & -- & (a) & -_ & (a) & (a) \\
\hline 13 & -- & (a) & (a) & -- & -- & $\ldots$ & - & -- & - & (a) & $\ldots$ & (a) & -- & (a) & (a) \\
\hline 14 & - & (a) & (a) & -- & -- & -. & - & -- & -- & (a) & $\ldots$ & (a) & .- & (a) & (a) \\
\hline 15 & - & (a) & (a) & -- & - & - & $-\infty$ & -- & .. & (a) & - & (a) & $\infty$ & (a) & (a) \\
\hline 16 & -- & (a) & $(a)$ & $\ldots$ & -- & .- & - & -- & $\ldots$ & -- & -- & -- & -. & (a) & (a) \\
\hline 17 & $\ldots$ & (a) & (a) & -. & - & -. & -- & - & -. & ... & - & - & -. & (a) & (a) \\
\hline 18 & -- & (a) & (a) & -. & -- & -- & $=$ & -- & -. & $-\infty$ & - & - & -- & (a) & (a) \\
\hline 19 & - & (a) & (a) & -- & -- & -. & - & $\ldots$ & -. & $\ldots$ & .. & - & $\ldots$ & (a) & $(a)$ \\
\hline 20 & - & (a) & (a) & .. & $\ldots$ & -- & $-\infty$ & -- & - & -- & -- & - & -- & (a) & (a) \\
\hline 21 & -- & (a) & (a) & -- & - & -. & -- & $\ldots$ & -- & -- & -. & -- & -. & (a) & (a) \\
\hline 22 & - & (a) & (a) & -- & -- & $\ldots$ & - & - & -- & $\ldots$ & - & - & - & (a) & (a) \\
\hline 23 & -. & (a) & (a) & .- & -- & -- & $\ldots$ & -- & -. & $\ldots$ & -- & -- & -- & (a) & $=$ \\
\hline 24 & - & (a) & (a) & -- & -- & - & -- & - & -.. & -. & - & -. & -- & (a) & - \\
\hline 25 & -- & (a) & (a) & -- & -- & & -. & $\ldots$ & - & - & -- & .02 & .01 & (b) & -. \\
\hline 26 & .03 & (b) & (b) & (c) & -- & -- & .22 & .14 & .22 & .40 & .15 & .20 & .05 & (b) & .10 \\
\hline 27 & .03 & (b) & (b) & .01 & .10 & .07 & .25 & .15 & .17 & .09 & .25 & $\ldots$ & .01 & (b) & .12 \\
\hline 28 & - & 8.10 & $d_{.08}$ & - & .02 & 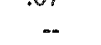 & .02 & - & .01 & .01 & - & .02 & - & 8.50 & $\ldots$ \\
\hline 29 & .. & (a) & (a) & - & $=$ & - & - & - & - & -. & - & - & .- & (a) & -- \\
\hline 30 & $\ldots$ & (a) & (a) & -- & $\ldots$ & $\ldots$ & - & -- & - & $\ldots$ & - & - & .. & (a) & -- \\
\hline 31 & -- & (a) & (a) & -- & -- & -- & -. & - & -- & -- &.- & - & -- & (a) & -. \\
\hline Total & 0.51 & $e_{1.03}$ & $e_{0.56}$ & 0.79 & 0.78 & 0.54 & 1.15 & 1.16 & $0.81 d$ & 0.73 & $0.88^{e}{ }_{1}$ & 1.07 & 0.39 & $e_{1.00}$ & 0.57 \\
\hline
\end{tabular}


TABLE 7.--Precipitation data from Weather Service Nuclear Support Office network stations--Continued.

\begin{tabular}{|c|c|c|c|c|c|c|c|c|c|c|c|c|c|c|c|}
\hline \multirow[b]{2}{*}{ DAY } & \multicolumn{15}{|c|}{ Station } \\
\hline & MER & $\mathbb{R V}$ & $4 \mathrm{JA}$ & DRA & $\mathrm{CS}$ & W5B & MV & $\mathrm{UCC}$ & $40 \mathrm{MN}$ & TS2 & BJY & $\mathrm{A} 12$ & PHS & PM1 & $\mathrm{LF}_{2}$ \\
\hline \multicolumn{16}{|c|}{ FEBRUARY 1985} \\
\hline 1 & -. & (b) & (b) & -- & -- & -- & 0.02 & 0.02 & 0.01 & -- & 0.01 & 0.10 & -. & (b) & -- \\
\hline 2 & - & (b) & (b) & -- & - & - & -- & -. & .02 & 0.04 & .04 & .08 & 0.06 & (b) & 0.01 \\
\hline 3 & 0.02 & $g_{0.02}$ & 80.02 & -- & -- & -- & .01 & -- & .01 & .01 & -- & .02 & - & 80.10 & .01 \\
\hline 4 & -- & (a) & (a) & -- & -- & -- & -- & -- & - & - & -- & - & .01 & (a) & -- \\
\hline 5 & -- & (a) & (a) & -- & -- & - & $\infty$ & $-\infty$ & -- & - & -- & - & 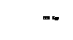 & (a) & -- \\
\hline 6 & -. & (a) & (a) & - & - & -- & - & -- & - & -. & -- & - & -. & (a) & -- \\
\hline 7 & -. & (a) & (a) & -. & -. & -- & -- & $\ldots$ & -. & -- & - & -- & .. & (a) & -- \\
\hline 8 & -. & $(a)$ & (a) & -- & -. & -. & .. & - & -- & -. & -- & -. & -. & (a) & -- \\
\hline 9 & -- & (b) & (b) & 0.02 & 0.03 & 0.05 & .19 & .01 & .24 & .15 & .10 & (b) & .17 & (b) & .05 \\
\hline 10 & - & 8.03 & $g .03$ & - & -- & -- & -- & - & .01 & -- & - & $f_{.21}$ & $-\infty$ & $g .10$ & -- \\
\hline 11 & -- & (a) & (a) & $\cdots$ & -- & -- & -- & - & -- & -. & - & -- & - & (a) & - \\
\hline 12 & - & (a) & (a) & - & -- & $=$ & -- & -. & -. & -- & -- & -. & - & (a) & -- \\
\hline 13 & -. & (a) & (a) & -- & -- & -- & -- & - & -- & -. & -- & - & $\cdots$ & (a) & .- \\
\hline 14 & - & (a) & (a) & - & -- & $\cdots$ & -- & - & -- & -- & - & - & -- & (a) & - \\
\hline 15 & $\infty$ & (a) & (a) & -- & - & - & -- & - & -- & - & - & $=$ & -- & (a) & - \\
\hline 16 & -- & (a) & (a) & -- & -- & -. & -- & - & -- & -. & -- & -- & -. & (a) & -- \\
\hline 17 & - & (a) & (a) & - & -- & -. & -- & $\cdots$ & -. & -- & - & - & $=$ & (a) & -- \\
\hline 18 & -. & (a) & (a) & - & - & -- & - & - & $\infty$ & -- & -- & - & -- & (a) & - \\
\hline 19 & -- & (a) & (a) & -- & -- & -- & -- & -- & - & -- & - & -- & -- & (a) & - \\
\hline 20 & -. & $(a)$ & (a) & (c) & -- & -. & -- & - & -- & -. & -- & -- & -. & (a) & - \\
\hline 21 & -- & (a) & (a) & -- & -- & -- & -- & - & -- & -- & -- & -- & -- & (a) & -- \\
\hline 22 & - & (a) & (a) & - & - & -. & -- & - & $=$ & -- & -- & $-\infty$ & -- & (a) & -- \\
\hline 23 & -- & (a) & (a) & - & -- & -- & -- & - & -- & -- & - & -- & -- & (a) & -- \\
\hline 24 & -- & $(a)$ & (a) & - & - & $-\cdot$ & -- & - & -- & -- & - & - & -- & (a) & -- \\
\hline 25 & -- & (a) & (a) & - & - & -- & - & - & - & - & -- & - & $\cdots$ & (a) & -- \\
\hline 26 & -- & (a) & (a) & $\ldots$ & -- & $\cdots$ & -- & - & -- & -- & - & - & -- & $(a)$ & -- \\
\hline 27 & -- & -- & $-\infty$ & -- & - & -- & -- & - & -- & $=$ & -- & - & -- & (a) & -- \\
\hline 28 & -- & -- & -- & -- & -- & - & -- & - & - & - & -- & - & -- & (a) & -. \\
\hline Total & 0.02 & $e_{0.05}$ & $e_{0.05}$ & 0.02 & 0.03 & 0.05 & 0.22 & 0.03 & 0.29 & 0.20 & 0.15 & 0.41 & 0.24 & ${ }^{e} 0.20$ & 0.07 \\
\hline
\end{tabular}


TABLE 7.--Precipitation data from Weather Service Nuclear Support Office network stations--Continued.

\begin{tabular}{|c|c|c|c|c|c|c|c|c|c|c|c|c|c|c|c|}
\hline \multirow[b]{2}{*}{ DAY } & \multicolumn{15}{|c|}{ Station } \\
\hline & MER & RV & $4 \mathrm{JA}$ & DRA & $\mathrm{Cs}$ & W5B & MV & UCC & $40 \mathrm{MN}$ & TS2 & BJY & $\mathrm{A} 12$ & PHS & PM1 & $\mathrm{LF}^{2}$ \\
\hline \multicolumn{16}{|c|}{ MARCH 1985} \\
\hline 1 & -- & -- & - & -- & -- & -- & -- & -- & -- & -- & -- & -- & -- & $(a)$ & -. \\
\hline 2 & -- & -. & 0.03 & -- & 0.02 & -. & 0.05 & 0.01 & -- & 0.02 & 0.01 & 0.07 & -- & (a) & .. \\
\hline 3 & -- & -- & -- & -- & -- & -. & -- & -- & -- & -. & - & -. & -. & (a) & .- \\
\hline 4 & -- & - & -- & -. & -- & -. & -- & - & -. & -. & - & -- & -. & (a) & - \\
\hline 5 & -- & -- & -- & -- & -- & -- & -. & -- & - & -- & -- & -- & -. & (a) & - \\
\hline 6 & .. & -- & .. & .- & -. & -- & -- & .. & -- & .. & -- & -. & -. & (a) & -. \\
\hline 7 & -- & -- & -- & -- & - & -- & -- & -- & -- & -- & -. & -- & -- & - & - \\
\hline 8 & -- & - & -- & -- & -- & -- & -. & .- & .- & .- & .- & -- & .- & .- & -- \\
\hline 9 & -- & -- & -- & -- & -- & -- & - & -- & - & -. & -. & -- & .. & -- & -- \\
\hline 10 & -- & - & -- & -. & - & -- & -- & -- & - & -. & -- & - & .. & -- & - \\
\hline 11 & .. & .. & -- & .. & - & -- & .01 & -- & -- & .01 & .. & .08 & .. & -. & 0.02 \\
\hline 12 & -- & - & -. & -- & -- & .- & -- & -- & 0.02 & -. & -- & -- & .- & -- & .02 \\
\hline 13 & .. & .- & -- & -. & -- & -- & -. & -- & - & .. & -- & -- & -- & -- & -- \\
\hline 14 & -- & -. & -- & -. & -- & -- & -- & -- & -- & -. & -- & -- & -. & -- & -- \\
\hline 15 & -- & $\cdots$ & -. & -- & -- & -- & -- & .- & -- & .. & -- & -- & .- & 0.06 & .- \\
\hline 16 & -- & -. & - & -- & .01 & -- & .08 & .06 & .13 & .. & .. & .02 & -. & .03 & .04 \\
\hline 17 & -- & -- & -- & -- & -. & .- & .01 & .. & .01 & .. & -- & -- & .. & -- & .01 \\
\hline 18 & 0.06 & 0.05 & - & 0.02 & .15 & 0.07 & .05 & .07 & .10 & .10 & .17 & .41 & 0.29 & .22 & .15 \\
\hline 19 & -- & - & .. & - & -- & -- & -- & -- & -- & -- & -- & .02 & -. & .09 & -- \\
\hline 20 & .- & .- & -- & -. & -- & -- & -- & -- & - & -. & -- & -- & .. & -- & - \\
\hline 21 & -. & .. & -. & -. & -- & -- & -- & -- & - & -. & -- & - & -. & -- & -- \\
\hline 22 & -. & .- & -- & -- & -. & -- & -- & -- & -. & - & -- & -. & - & .- & - \\
\hline 23 & -. & -. & -- & -- & -- & - & -- & -- & -- & -- & -- & -- & -- & -- & -. \\
\hline 24 & .- & .- & -- & -- & .. & .- & .- & .- & .- & .- & .. & .- & .- & .- & -. \\
\hline 25 & -- & -- & - & -. & -- & - & -- & -- & - & -- & -. & -- & -- & - & - \\
\hline 26 & .- & .. & .- & .- & .. & -- & -- & .. & .. & -. & .. & -- & ... & .- & -. \\
\hline 27 & -- & .. & .- & .- & .- & -- & -- & -. & .. & .. & .. & -- & -- & .- & .. \\
\hline 28 & .11 & .02 & .08 & .06 & .03 & .03 & .04 & .05 & .05 & .03 & .03 & .06 & -- & .01 & .03 \\
\hline 29 & -. & -- & -- & -- & .. & - & -- & -- & -- & - & -- & -- & -- & .- & -. \\
\hline 30 & -- & .- & -- & - & -- & -. & -. & -. & -. & -- & -. & -. & -- & -- & - \\
\hline 31 & -- & -. & - & -- & $\cdots$ & $=$ & -- & -- & -- & - & -- & - & -- & -. & - \\
\hline Total & 0.17 & 0.07 & 0.11 & 0.08 & 0.21 & 0.10 & 0.24 & 0.19 & 0.31 & 0.16 & 0.21 & 0.66 & 0.29 & 0.41 & 0.27 \\
\hline
\end{tabular}


TABLE 7.--Precipitation data from Weather Service Nuclear Support Office network stations--Continued.

\begin{tabular}{|c|c|c|c|c|c|c|c|c|c|c|c|c|c|c|c|}
\hline \multirow[b]{2}{*}{ DAY } & \multicolumn{15}{|c|}{ Station } \\
\hline & MER & RV & $4 \mathrm{JA}$ & DRA & CS & W5B & MV & UCC & $40 \mathrm{MN}$ & TS2 & BJY & A12 & PHS & PM1 & LF2 \\
\hline \multicolumn{16}{|c|}{ APRIL 1985} \\
\hline 1 & -- & - & -- & -- & -- & -- & -- & -- & -- & -. & -- & -- & -- & -- & - \\
\hline 2 & -- & -- & -- & -- & -- & -- & -- & -- & -- & -. & -- & -- & -- & -- & -. \\
\hline 3 & -- & -- & -- & -- & -- & -- & -- & -- & -- & -- & -- & -- & -- & -- & -- \\
\hline 4 & -- & -- & -- & -- & -- & -- & -- & -- & -- & -- & -- & -- & -- & -- & - \\
\hline 5 & - & -- & -- & -- & -- & -- & - & -- & -- & -- & -- & -- & -- & -- & - \\
\hline 6 & -- & -- & -- & -- & -. & -- & -- & -- & -- &.- & -- & -- & -- & -- & - \\
\hline 7 & -- & -- & -. & -- & -- & -- & -. & -- & -- & -. & -- & -- & -- & -- & - \\
\hline 8 & -- & -- & -- & -- & -- & -- & -- & -- & - & -- & -- & -- & -- & -. & -- \\
\hline 9 & -- & -- & -- & -. & -- & -- & -. & -- & -- & -- & -- & -- & -- & -- & -. \\
\hline 10 & -- & -- & -- & -- & -- & -- & -- & -- & - & -- & -- & -- & -- & -- & -- \\
\hline 11 & -- & -- & -- & -- & -- & -. & -- & -- & -- & -- & -- & .. & -- & -- & - \\
\hline 12 & -. & -- & -- & -- & -- & -- & -- & -- & -- & -- & -- & -- & -- & -- & - \\
\hline 13 & -- & -- & -- & -- & -- & -- & -. & -- & -- & -- & -- & -- & -- & -- & - \\
\hline 14 & -- & .. & -- & -. & .- & -. & -. & .. & - & -- & -- & -- & -- & -. & - \\
\hline 15 & -- & -- & - & -- & -- & -- & -- & -- & -- & -- & -- & -- & -- & -- & - \\
\hline 16 & -. & -- & -- & -- & -- & -- & -- & -- & -- & .. & -. & -- & -- & -- & $-\infty$ \\
\hline 17 & -. & -- & -- & -- & .- & -- & -- & -- & -- & -- & -- & -- & -- & -- & -. \\
\hline 18 & -- & -- & -- & 0.02 & -. & -- & -- & -- & -- & -- & -- & -- & -- & -- & - \\
\hline 19 & -- & .- & -. & -- & -. & -- & -. & -- & -- & -- & -- & -. & - & -- & - \\
\hline 20 & -- & - & - & -. & -- & - & -. & - & -- & -- & - & -- & - & -- & - \\
\hline 21 & -- & -- & -- & -- & -- & -- & .. & -- & -. & -- & -.. & -. & -- & .- & -. \\
\hline 22 & .- & -- & -- & -- & -- & -. & .. & -- & -- & -- & -. & -- & -- & -- & -- \\
\hline 23 & -- & -- & -- & -- & - & -- & -- & -- & -- & - & -- & -- & - & -- & -- \\
\hline 24 & -- & -- & -- & -. & -- & -- & -. & -- & -- & -. & -- & .- & -- & -- & - \\
\hline 25 & 0.02 & 0.01 & -- & (c) & -- & 0.01 & -. & 0.01 & 0.03 & - & 0.01 & 0.02 & 0.01 & 0.01 & 0.01 \\
\hline 26 & -. & -- & -- &.- & -- & -- & -. & -- & -- & - & - & -- & -. & -- & -- \\
\hline 27 & .- & -- & -- & .- & -- & -- & $=$ & -- & .. & - & -. & -- & -- & -- & - \\
\hline 28 & .- & .. & -- & -- & -- & .- & -- & -- & -- & -- & -- & -- & - & -. & -- \\
\hline 29 & -. & -- & -. & -- & -- & -- & -. & -- & -- & -- & -. & -- & -- & -- & -- \\
\hline 30 & -- & -- & -- & -- & -- & -- & -. & -- & -. & -- & -- & -- & -- & - & -- \\
\hline Total & 0.02 & 0.01 & 0 & 0.02 & 0 & 0.01 & 0 & 0.01 & 0.03 & 0 & 0.01 & 0.02 & 0.01 & 0.01 & 0.01 \\
\hline
\end{tabular}


TABLE 7.--Precipitation data from Weather Service Nuclear Support Office network stations--Continued.

\begin{tabular}{|c|c|c|c|c|c|c|c|c|c|c|c|c|c|c|c|}
\hline \multirow[b]{2}{*}{ DAY } & \multicolumn{15}{|c|}{ Station } \\
\hline & MER & RV & 4JA & DRA & $\mathrm{CS}$ & W5B & MV & $\mathrm{UCC}$ & $40 \mathrm{MN}$ & $\mathrm{TS} 2$ & BJY & A12 & PHS & PM1 & LF2 \\
\hline \multicolumn{16}{|c|}{ MAY 1985} \\
\hline 1 & - & $\ldots$ & -. & - & -- & $-\infty$ & -- & - & -- & -- & $=$ & $\ldots$ & $-\infty$ & -. & - \\
\hline 2 & -- & -- & -- & -- & - & - & -- & -- & - & -- & - & -- & .. & - & - \\
\hline 3 & -- & $-\infty$ & -- & -. & - & -- & $-\infty$ & -- & -- & -- & - & - & .. & - & -- \\
\hline 4 & -- & -. & -- & -- & -- & - & $\cdots$ & -- & -- & -- & -- & -- & -- & -- & - \\
\hline 5 & -- & -- & -- & -- & $-\infty$ & -- & - & - & - & -- & - & -- & -- & - & - \\
\hline 6 & -- & -. & - & - & - & - & - & -- & -- & -- & -- & -. & -. & -- & -- \\
\hline 7 & -- & -- & -. &.- & -- & -- & -- & - & - & -- & -- & -- & -- & -- & -- \\
\hline 8 & -- & $\ldots$ & -. & .. & -- & -. & .. & -- & -- & -- & -- & -- & $=$ & -. & -- \\
\hline 9 & 0.04 & 0.07 & -- & 0.08 & 0.09 & 0.02 & 0.09 & 0.05 & 0.01 & 0.01 & 0.03 & -. & -- & 0.03 & 0.02 \\
\hline 10 & .06 & .20 & 0.21 & .12 & .25 & .05 & .23 & .07 & .30 & .28 & .16 & 0.19 & 0.25 & .05 & .24 \\
\hline 11 & -- & -- & -- & -- & -- & -. & -- & - & $\ldots$ & - & -- & -- & $\ldots$ & -- & -- \\
\hline 12 & -. & - & -. & .. & .- & .. & .. & -- & -- & -- & $\infty$ & -. & .- & -- & -- \\
\hline 13 & -- & -- & - & -. & -- & -- & - & -- & -- & -- & -- & -- & -- & -. & -- \\
\hline 14 & -- & -- & -- & -- & -- & -. & - & -- & -- & - & $\ldots$ & -- & .. & -- & -- \\
\hline 15 & -- & - & - & -- & - & -- & $-\infty$ & -- & -- & -- & -- & -- & -- & - & - \\
\hline 16 & - & -- & - & -- & -- & -- & -- & -- & -- & -- & $=$ & -- & -. & -- & -- \\
\hline 17 & -- & -- & - & -- & -- & -. & $\cdots$ & -- & -- & -- & - & -- & -. & -- & - \\
\hline 18 & - & -- & -- & -- & - & -- & -- & -- & -- & -- & -- & - & -- & -- & - \\
\hline 19 & - & -- & -- & $-\infty$ & -- & - & - & -- & -- & - & -- & - & - & -- & -- \\
\hline 20 & -- & - & -- & -. & 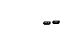 & -- & $\ldots$ & -- & - & -- & -- & -- & -- & -- & - \\
\hline 21 & - & - & - & -- & -- & -. & -- & - & -- & -- & - & -- & .- & ... & -- \\
\hline 22 & -- & -. & - & $\ldots$ & -- & $\ldots$ & $\ldots$ & -- & -- & -. & $\ldots$ & - & - & $\infty$ & -. \\
\hline 23 & -- & - & - & $-m$ & -- & -- & -- & -- & -- & -- & - & -- & -- & -- & - \\
\hline 24 & - & -- & -- & -- & -- & - & $\ldots$ & -- & -- & -- & - & - & -- & - & -. \\
\hline 25 & - & - & - & - & -. & -- & - & - & $\cdots$ & -- & - & -- & -- & - & -- \\
\hline 26 & -- & -- & -- & - & -- & -. & -- & -- & -- & -. & -- & -- & -- & -- & -- \\
\hline 27 & -- & $=$ & - & $-\infty$ & -- & -- & -- & - & -. & -- & - & -- & $-\infty$ & $\ldots$ & -. \\
\hline 28 & - & -- & -- & -- & -- & - & -- & -- & .- & -. & -- & -- & -- & -- & -- \\
\hline 29 & -- & -. & - & -- & $-\infty$ & -. & -. & -- & -- & -- & - & -- & -- & -- & -- \\
\hline 30 & -- & -- & - & - & -- & -- & -- & -- & -- & -- & -- & - & -- & -- & -- \\
\hline 31 & - & - & -- & $\ldots$ & - & -- & -- & - & -- & -- & $-\infty$ & -- & - & -- & -- \\
\hline Total & 0.10 & 0.27 & 0.21 & 0.20 & 0.34 & 0.07 & 0.32 & 0.12 & 0.31 & 0.29 & 0.19 & 0.19 & 0.25 & 0.08 & 0.26 \\
\hline
\end{tabular}


TABLE 7.--Precipitation data from Weather Service Nuclear Support Office network stations--Continued.

\begin{tabular}{|c|c|c|c|c|c|c|c|c|c|c|c|c|c|c|c|}
\hline \multirow[b]{2}{*}{ DAY } & \multicolumn{15}{|c|}{ Station } \\
\hline & MER & RV & 4JA & DRA & CS & W5B & MV & UCC & $40 \mathrm{MN}$ & TS2 & BJY & A 12 & PHS & PM1 & LF2 \\
\hline \multicolumn{16}{|c|}{ JUNE 1985} \\
\hline 1 & .- & .- & .- & -- & -- & -- & .. & -- & .- & .. & .. & -. & .- & (a) & -. \\
\hline 2 & -- & .. & -. & .- & .- & (b) & -- & 0.10 & (b) & 0.05 & 0.04 & 0.04 & $\ldots$ & (b) & .03 \\
\hline 3 & -- & 0.02 & 0.03 & (c) & 0.05 & $f_{0.10}$ & 0.05 & .04 & $f_{0.10}$ & .11 & .27 & .39 & 0.35 & $f_{0.37}$ & 0.13 \\
\hline 4 & .- & -. & -. & $\ldots$ & -- & .- & -- & -- & -. & -- & -- & -- & -. & (a) & -. \\
\hline 5 & -- & -. & .- & -- & - & -. & -- & -- & -- & -- & -- & -. & -- & (a) & -- \\
\hline 6 & -- & -- & .. & -- & -- & -- & -- & -- & -. & -- & -- & -- & -- & (a) & -- \\
\hline 7 & -- & .- & -. & -- & -- & -- & -- & -- & -- & -- & -- & -- & -- & (a) & -- \\
\hline 8 & - & -- & -- & -- & - & -- & -- & -- & -- & -- & -- & -- & -. & (a) & -. \\
\hline 9 & - & -- & -- & -- & -- & - & -- & -- & -- & -- & -- & -- & -. & (a) & -. \\
\hline 10 & -. & .- & .. & - & -- & - & .- & - & -- & -. & -. & -- & -. & (a) & -. \\
\hline 11 & -- & -- & -- & -- & -- & -- & .- & -- & -- & -- & -- & -- & -- & (a) & -- \\
\hline 12 & .. & .. & .. & -- & -- & -- & -- & -- & -- & .- & .- & -- & -- & (a) & .- \\
\hline 13 & -- & -- & -. & -- & -- & .. & .- & -- & .- & .- & -- & -- & -- & (a) & -- \\
\hline 14 & -- & -. & .. & .. & -- & -- & .- & -- & .- & -- & -- & -- & -- & (a) & .- \\
\hline 15 & -- & -. & .. & -.. & -- & -- & .. & .- & -- & .. & .- & -- & -.- & (a) & -- \\
\hline 16 & .. & .. & .. & .. & -. & -- & .. & -- & .. & .- & .- & .- & -- & (a) & .- \\
\hline 17 & -- & .. & .. & .. & .- & -- & -- & -- & -- & .- & -. & -- & .- & (a) & .. \\
\hline 18 & -- & -- & -- & -- & - & -- & -- & -- & -- & -. & -- & -- & -- & (a) & -- \\
\hline 19 & -- & -- & -- & -- & -- & -- & -- & -- & -- & .- & -. & - & -- & (a) & -- \\
\hline 20 & -. &.- & -- & -- & -. & -- & -- & - & -- & -- & .- & $\ldots$ & -. & (a) & -- \\
\hline 21 & -- & .- & .- & -- & -- & . & -- & -- & -- & .. & -- & -- & -- & (a) & -- \\
\hline 22 & -- & -. & -- & .. & -. & -- & -- & -- & -- & -- & -- & -- & -- & (a) & -- \\
\hline 23 & - & $\cdots$ & -. & -- & -- & - & -- & -- & -- & -. & -- & - & -- & (a) & -- \\
\hline 24 & -- & .. & .. & -- & .01 & .01 & .03 & .03 & .01 & -- & .05 & .04 & .03 & .04 & .02 \\
\hline 25 & -- & .. & -- & -- & - & -. & -. & -- & -- & .- & $-\infty$ & -- & -- & (a) & -- \\
\hline 26 & .. & .- & .. & .- & .- & -. & .- & .. & .. & .- & .- & .- & -. & (a) & -- \\
\hline 27 & - & -. & -- & -- & .- & .. & .- & - & .- & -- & -- & - & -- & (a) & -- \\
\hline 28 & -- & ... & .. & -- & -- & -- & -- & -- & $\ldots$ & .. & .- & .. & .. & (a) & -- \\
\hline 29 & -- & .- & -. & -- & -- & -. & .. & -. & -- & -- & -. & -- & .. & (a) & -- \\
\hline 30 & -. & .- & .- & - & - & -- & -. & .- & -. & -. & .. & -. & -. & (a) & -- \\
\hline Total & 0 & 0.02 & 0.03 & (c) & 0.06 & 0.11 & 0.08 & 0.17 & 0.11 & 0.16 & 0.36 & 0.47 & 0.38 & 0.41 & 0.18 \\
\hline
\end{tabular}


TABLE 7.--Precipitation data from Weather Service Nuclear Support Office network stations--Continued.

\begin{tabular}{|c|c|c|c|c|c|c|c|c|c|c|c|c|c|c|c|}
\hline \multirow[b]{2}{*}{ DAY } & \multicolumn{15}{|c|}{ Station } \\
\hline & MER & RV & $4 \mathrm{JA}$ & DRA & $\mathrm{CS}$ & W5B & $\mathrm{MV}$ & UCC & $40 \mathrm{MN}$ & TS2 & BJY & A12 & PHS & PM1 & LF2 \\
\hline \multicolumn{16}{|c|}{ JULY 1985} \\
\hline 1 & -. & -. & $-\infty$ & - & $\infty$ & -- & -- & - & - & -. & $\ldots$ & - & -. & (a) & -- \\
\hline 2 & -- & $\ldots$ & -- & -- & $\cdots$ & - & - & -- & $\ldots$ & - & -- & -- &.- & (a) & -- \\
\hline 3 & - & -- & - & - & -- & -- & -- & -- & -- & -- & -- & -- & -- & (a) & -- \\
\hline 4 & - & -- & - & - & -- & $=-$ & -- & -- & -- & -. & -. & -- & -- & (a) & -- \\
\hline 5 & -- & -- & -- & -- & - & -- & -- & -- & - & -- & - & -- & -- & (a) & -- \\
\hline 6 & $-\infty$ & -. & -- & - & -- & -. & -- & -- & - & - & ... & 0.02 & -- & (b) & -- \\
\hline 7 & -- & -- & - & -- & -- & -- & $-\infty$ & -- & -- & -- & 0.29 & -- & -- & (b) & -- \\
\hline 8 & -- & -- & -- & - & .. & $=$ & -- & -. & -. & -- & -. & -. & -- & $f_{0.02}$ & -. \\
\hline 9 & -- & -- & -- & -- & -- & -- & -- & -.. & -- & -- & -- & - & -- & (a) & -- \\
\hline 10 & -- & -- & - & -- & -- & -- & -- & -- & - & -- & -- & - & -- & (a) & - \\
\hline 11 & $\infty$ & -- & -- & -- & -- & -- & - & -- & - & - & - & - & -- & $(a)$ & - \\
\hline 12 & -- & -- & -. & -- & -- & .. & -. & -- & -- & -- & -- & -- & -- & (a) & -- \\
\hline 13 & -- & -- & -- & -- & -- & -- & -- & -- & -- & -- & -- & -- & -- & (a) & -- \\
\hline 14 & -. & -. & -. & -- & - & $=$ & - & -- & -- & -- & -- & -- & - & (a) & -- \\
\hline 15 & - & -- & -- & -- & - & - & -- & -- & - & -- & - & -- & -- & (a) & -- \\
\hline 16 & - & -. & $\ldots$ & -- & -- & - & -- & -. & $-\infty$ & $\infty$ & -- & -- & - & (a) & -- \\
\hline 17 & - & -- & -- & -- & (b) & (b) & -. & -- & -- & -- & -. & .16 & - & (a) & -- \\
\hline 18 & 0.17 & (b) & 0.23 & 0.01 & (b) & (b) & 0.28 & 0.15 & 0.50 & .50 & .13 & .28 & 0.14 & 0.11 & 0.21 \\
\hline 19 & - & (b) & .- & (c) & (b) & (b) & .18 & .08 & .21 & -. & .10 & .87 & .08 & .29 & .29 \\
\hline 20 & .16 & (b) & .13 & .12 & (b) & (b) & .60 & .22 & 1.27 & .08 & .22 & .65 & .06 & .13 & .13 \\
\hline 21 & $\ldots$ & $f_{0.94}$ & .01 & .25 & $f_{1.25}$ & $f_{1.07}$ & .04 & .08 & .02 & - & .10 & .02 & -- & .02 & .01 \\
\hline 22 & -- & .- & -- & -- & - & -- & -. & -- & -- & - & -. & -- & -- & -. & -- \\
\hline 23 & -. & -- & -- & -- & -- & -- & -- & -- & -- & - & -- & - & .01 & -- & -- \\
\hline 24 & - & -. & -- & -- & -- & -. & -- & -. & $-\infty$ & -- & - & -. & -- & -- & -- \\
\hline 25 & - & -- & -- & -- & - & -- & -- & -- & -- & -- & -- & - & - & -- & -- \\
\hline 26 & $=$ & - & -. & .. & - & - & .- & - & -- & $=$ & - & $-\infty$ & -- & -- & -. \\
\hline 27 & - & -. & -. & -- & -- & -- & -- & -- & -- & -- & -- & -. & -- & -- & -- \\
\hline 28 & -- & -- & .. & -- & -. & -- & -- & - & $=-$ & -- & .. & $=$ & - & -- & -- \\
\hline 29 & - & -- & -- & -- & -- & - & -. & -- & -- & - & -- & - & - & - & -- \\
\hline 30 & -- & -- & -. & -- & -- & -- & -- & -. & -. & - & -- & -. & - & - & .. \\
\hline 31 & - & - & - & -- & -- & -- & - & -- & -- & - & - & -- & -- & -- & -- \\
\hline Total & 0.33 & 0.94 & 0.37 & 0.38 & 1.25 & 1.07 & 1.10 & 0.53 & 2.00 & 0.58 & 0.84 & 2.00 & 0.29 & 0.57 & 0.64 \\
\hline
\end{tabular}


TABLE 7.--Precipitation data from Weather Service Nuclear Support Office network stations--Continued.

\begin{tabular}{|c|c|c|c|c|c|c|c|c|c|c|c|c|c|c|c|}
\hline \multirow[b]{2}{*}{ DAY } & \multicolumn{15}{|c|}{ Station } \\
\hline & MER & $\mathbf{R V}$ & $4 \mathrm{JA}$ & DRA & Cs & W5B & MV & $\mathrm{UCC}$ & $40 \mathrm{MN}$ & TS2 & BJY & A12 & PHS & PM1 & $\mathrm{LF} 2$ \\
\hline \multicolumn{16}{|c|}{ AUGUST 1985} \\
\hline 1 & -- & -- & -- & -- & -- & -- & -- & -- & -- & -- & - & -- & -- & -- & -- \\
\hline 2 & -- & -- & -- & -- & - & -. & -- & -- & -- & -- & - & -- & -- & -- & -- \\
\hline 3 & -- & -- & -- & -- & -- & -. & -- & -- & -- & - & -- & -- & -- & -- & -- \\
\hline 4 & $\cdots$ & -. & -- & -- & $\cdots$ & -- & -. & -- & .- & -- & -- & -. & -- & -- & -- \\
\hline 5 & -- & -- & -- & -- & -- & -. & - & - & -- & -- & -- & -- & -- & -- & -- \\
\hline 6 & -- & -- & -- & -- & -- & -- & -- & -- & .. & -- & -. & -- & -- & - & -. \\
\hline 7 & -- & -. & -- & -- & -- & -- & -- & -- & -- & -- & -- & -.. & -. & -- & -- \\
\hline 8 & -- & -. & -- & -- & -- & -- & -- & -- & -- & -- & -- & -- & -- & -- & -- \\
\hline 9 & -- & -- & -. & -- & -- & -- & -- & -- & -- & -- & -- & -- & -- & -- & -- \\
\hline 10 & -- & - & -- & - & -- & -- & -- & -- & -- & - & - & -- & -- & -- & - \\
\hline 11 & .- & -- & -- & -- & .. & -- & -- & -- & -- & -- & - & -- & .. & -- & -- \\
\hline 12 & -- & -. & .- & -- & -. & - & -- & -- & -- & $=-$ & -- & -- & -- & -- & -- \\
\hline 13 & -- & - & -- & -- & -- & -- & - & - & -- & - & -- & -- & -- & -- & -- \\
\hline 14 & -. & -- & -. & -. & -- & -- & .. & -- & -. & -. & - & -- & -- & -- & -- \\
\hline 15 & -- & -- & -- & -- & -- & -- & -- & -- & -- & -- & -. & -- & -. & -- & - \\
\hline 16 & -- & -- & -- & -- & - & .. & -. & -- & -- & .- & -- & .. & -- & -. & -- \\
\hline 17 & -. & -- & -- & -- & $=$ & -- & -- & -- & -. & -- & -. & -- & -- & -- & - \\
\hline 18 & -- & -- & -- & -. & -- & -- & -- & -- & - & $\ldots$ & -- & -- & - & -. & - \\
\hline 19 & .- & -- & .- & -- & - & -- & -- & .- & .- & -. & -. & -- & -. & .- & - \\
\hline 20 & -- & -- & -- & -- & -- & -- & -. & -- & - & .. & -- & -- & - & -- & -- \\
\hline 21 & .. & -- & -- & .- & - & .. & $\ldots$ & -- & .- & -- & -. & -. & -. & -- & .- \\
\hline 22 & .- & - & .. & -- & -. & -. & .. & -. & -- & -. & -- & - & -- & $=$ & -- \\
\hline 23 & -- & -- & -. & -- & - & -- & -. & -- & -- & -. & -. & -- & -- & -. & - \\
\hline 24 & -. & $=$ &. & -. & $=$ & -- & $=$ & -- & -- & -- & -. & - & -- & -- & - \\
\hline 25 & -. & -. & -. & -. & -- & - & -. & -- & -- & -- & -. & - & -- & -- & -- \\
\hline 26 & .- & -- & -- & -- & -- & -- & $=$ & -- & -- & .. & -- & -. & -- & $=$ & - \\
\hline 27 & -. & -- & -- & -. & -- & -. & - & - & -- & -- & -- & - & - & -- & -. \\
\hline 28 & .. & -- & -. & -- & -- & -. & -. & .. & -- & -. & -. & - & -- & -- & -. \\
\hline 29 & -. & -- & -- & .. & -- & -- & - & .. & - & -- & -. & - & - & - & $=$ \\
\hline 30 & -- & -- & -- & -. & -- & -- & -. & -. & -- & -. & -- & - & - & .. & -- \\
\hline 31 & -. & -- & -- & .- & - & - & - & .. & -- & .. & -- & -- & -- & -. & - \\
\hline Total & 0 & 0 & 0 & 0 & 0 & 0 & 0 & 0 & 0 & 0 & 0 & 0 & 0 & 0 & 0 \\
\hline
\end{tabular}


TABLE 7.--Precipitation data from Weather Service Nuclear Support Office network stations--Continued.

\begin{tabular}{|c|c|c|c|c|c|c|c|c|c|c|c|c|c|c|c|}
\hline \multirow[b]{2}{*}{ DAY } & \multicolumn{15}{|c|}{ Station } \\
\hline & MER & RV & 4IA & DRA & $\mathrm{CS}$ & W5B & MV & UCC & $40 \mathrm{MN}$ & TS2 & BIY & A12 & PHS & PM1 & LF2 \\
\hline \multicolumn{16}{|c|}{ SEPTEMBER 1985} \\
\hline 1 & $-\infty$ & $-\infty$ & $\ldots$ & -- & -- & -- & - & -- & -- & -. & - & -. & -- & - & - \\
\hline 2 & -- & - & -- & - & - & -- & - & -. & -- & -- & -- & -- & -. & - & - \\
\hline 3 & - & - & -- & - & - & $-\infty$ & - & $=$ & -- & - & - &.- & - & $\ldots$ & 0.03 \\
\hline 4 & $-\infty$ & - & - & - & -- & - & -- & -- & - & - & - & -- & - & $\cdots$ & -- \\
\hline 5 & - & -- & -- & -- & -- & - & -- & - & -- & - & - & -- & - & -- & -- \\
\hline 6 & 0.28 & 0.01 & - & 0.07 & - & -- & -- & 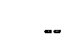 & -. & -- & .. & - & $\infty$ & .. & -- \\
\hline 7 & -- & (a) & -- & -- & -- & -- & -- & -- & -- & - & -- & -- & - & -- & -- \\
\hline 8 & -- & (a) & - & -- & -- & $\ldots$ & $-\infty$ & -- & -- & -- & -- & -. & -- & -. & -. \\
\hline 9 & -- & (a) & -- & - & - & -- & - & -. & -- & - & -- & $-\infty$ & $\ldots$ & $\ldots$ & -- \\
\hline 10 & - & (a) & -- & -- & - & -- & -- & -- & - & -- & -- & -- & - & -- & -- \\
\hline 11 & -- & (a) & -- & 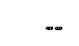 & - & -- & -- & - & $\ldots$ & -. & -- & -. & -. & -- & -- \\
\hline 12 & - & (a) & -- & -- & -- & -- & - & -- & -- & - & -. & -- & -. & -- & -- \\
\hline 13 & - & (a) & -- & - & - & -. & - & -- & - & -- & - & $-\infty$ & -. & -. & -- \\
\hline 14 & -- & (a) & -- & -. & -. & -- & - & -- & $\ldots$ & -- & - & $\ldots$ & -- & - & .- \\
\hline 15 & - & (a) & -- & -. & -- & $\ldots$ & - & -- & -- & -- & $\ldots$ & -- & -- & -- & -. \\
\hline 16 & - & (a) & -. & $\cdots$ & -- & - & -. & - & -. & .. & $\ldots$ & -- & $\ldots$ & -- & -. \\
\hline 17 & -. & (a) & -. & -- & - & -- & -- & -- & -. & -- & - & -- & -- & -- & .. \\
\hline 18 & .76 & (b) & 0.66 & 1.25 & 0.15 & 0.22 & 0.19 & 0.10 & 0.23 & 0.25 & 0.05 & 0.37 & 0.26 & 0.29 & .21 \\
\hline 19 & .05 & $f .76$ & -- & .09 & -- & -- & .02 & - & .02 & -- & -- & -- & -- & .01 & .02 \\
\hline 20 & - & -- & $\infty$ & -- & -. & -. & - & - & -. & -- & -- & -- & - & -- & -- \\
\hline 21 & -- & - & - & -. & -. & -. & - & -- & -- & -. & - & -. & -. & -. & 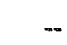 \\
\hline 22 & -- & -. & -- & -- & - & -. & - & - & -- & -. & - & -- & -- & -- & -- \\
\hline 23 & -- & -. & $=$ & $=$ & -. & -- & .- & -- & - & .. & - & -- & -- & -. & -. \\
\hline 24 & -- & -- & -. & .- & - & -- & -- & - & $\ldots$ & -- & -. & -- & .- & -- & -. \\
\hline 25 & $\cdots$ & - & $=$ & -- & - & -. & -- & $\ldots$ & - & -- & -- & -. & -- & -- & -- \\
\hline 26 & -- & -. & -- & -. & -. & -- & - & -- & -. & -- & -- & -- & -- & -. & .. \\
\hline 27 & .20 & .06 & .01 & .02 & .01 & .01 & .22 & .10 & .02 & - & .04 & .21 & .02 & .12 & .04 \\
\hline 28 & -- & - & -- & -- & - & -. & -- & -. & $\ldots$ & -. & - & -. & - & - & -. \\
\hline 29 & - & -- & -. & +- & -. & -- & -- & -- & $\ldots$ & -.. & -- & -- & .. & -. & - \\
\hline 30 & $=$ & -- & -- & .. & -. & -. & -. & -. & $-\infty$ & -. & - & .- & _- & .. & .- \\
\hline 31 & - & -. & -. & -- & - & -. & - & -- & -- & $\ldots$ & - & -. & - & -- & .. \\
\hline Total & 1.29 & 0.83 & 0.67 & 1.43 & 0.16 & 0.23 & 0.43 & 0.20 & 0.27 & 0.25 & 0.09 & 0.58 & 0.28 & 0.42 & 0.30 \\
\hline
\end{tabular}

a Daily precipitation probably zero (primary gage malfunction).

$b$ Daily precipitation included in cumulative total (primary gage malfunction).

c. Trace of precipitation.

$d$ Estimated cumulative total for this date and preceding adjacent days showing a $b$ (primary gage malfunction).

$e^{e}$ Monthly total estimated.

$f$ Cumulative total for this date and preceding adjacent days showing a $b$ (primary gage malfunction).

$g$ Subjective cumulative total for this date and preceding adjacent days showing a $b$ (all gages out). 